con-3288-35

HOPKINS COMPUTER RESEARCH REPORTS

REPORT 35

AUGUST 1974

\title{
AN ANALYSIS OF CONTROLLING AGENTS FOR \\ ASYNCHRONOUS PROCESSES
}

BY

TI LAK Agerwala

RESEARCH PROGRAM IN COMPUTER SYSTEMS ARCHITECTURE

Computer Science Program

THE JOHNS HOPKINS UNIVERSITY

Baltimore, Maryland

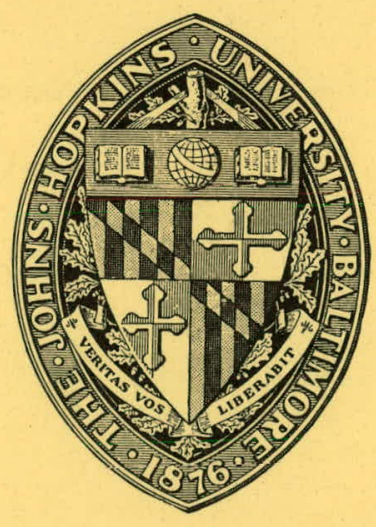




\section{DISCLAIMER}

This report was prepared as an account of work sponsored by an agency of the United States Government. Neither the United States Government nor any agency Thereof, nor any of their employees, makes any warranty, express or implied, or assumes any legal liability or responsibility for the accuracy, completeness, or usefulness of any information, apparatus, product, or process disclosed, or represents that its use would not infringe privately owned rights. Reference herein to any specific commercial product, process, or service by trade name, trademark, manufacturer, or otherwise does not necessarily constitute or imply its endorsement, recommendation, or favoring by the United States Government or any agency thereof. The views and opinions of authors expressed herein do not necessarily state or reflect those of the United States Government or any agency thereof. 


\section{DISCLAIMER}

Portions of this document may be illegible in electronic image products. Images are produced from the best available original document. 
An Analysis of Controlling Agents for Asynchronous Processes*

Tilak Agerwala

Electrical Engineering Dept.

The Johns Hopkins Univeristy

Baltimore, Maryland 21218

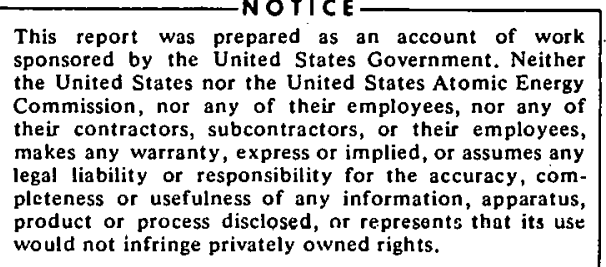
would not infringe privately owned rights.

*This work was supported by the U. S. Atomic Energy Commission under Contract AT (11-1 3288). 
AN ANALYSIS OF CONTROLLING AGENTS FOR ASYNCHRONOUS PROCESSES

T1lak Agerwala

August 1974

\section{Introduction}

The purpose of this report is to study different models of parallel computation and to analyze the capablities and limitations of the controlling agents in these mode1s. We are intcrested in determining, for each such controlling agent, whether it can represent all posstble types of interactions between a group of processes. If the answer is negative we wIlI try and determine exactly how powerful the model is. Our main interest is the study of control flow and its representation. The report will thus attempt to classify, according to capabilities, a large number of existing models. The approach will be systemat1c rather than ad hoc. We will define nine different classes of nets. Each class of nets represents a particular kind of control and 18 basically an ordinary Petrl net with slight modifications. Many of these classes have been Introduced and studied earlier, [2, 3, 13, 20], but we present those results here for the sake of completeness. We w11l establish a hferarchy of these nets based on their capability of representing interaction between processes; at one end are nets that are equivalent to finite state machines and at the other end, we have nets that can simulate arbitrary Turing machines. Establishing this hlerarchy of nets will systematize our study of different models since very often, the control part of a particular model will be in one to one correspondence with one of the nine classes. Numerous models of parallel computation have been proposed and studled. Usually such a study has been carried out independent of other models. Th1s paper is a first attempt at comparing these models formally and classifying them according to their modelling power. It is thus a step in the direction of establishing a uniform, underlying theory for the study of asynchronous systems.

\footnotetext{
*See important note page 84 .
} 
In a previous paper [2] we introduced the extended Petri net model and showed that it was complete [3], i.e., it could represent all possible kinds of Interaction between a group of processes and thus provided a correct, formal counterpart to the vague notion of "coordination". The current report establishes the significance of the extended Petri net model by demonstrating that most existing models of parallel computation are incomplete, and the extended Petri net model is one of the very few models that is of practical significance and not introduced for theoretical definitions alone.

Basically, any model for studying parallel systems consists of two. parts:

(1) A control mechanism, C, which decides whether a particular process is to be enabled or not.

(2) A set of asynchronous processes $P R=\left\{P_{1}, P_{2}, \ldots, P_{n}\right\}$ that are to be "coordinated".

If $C$. and PR are the only two entities specified, we have an uninterpreted model. In general, each process performs some computation. While studying the representation of coordinations, we are not concerned with the computation performed by the system as a whole, or that performed by each individual process. If the computation is of interest, additional specification must. be provided. Given an uninterpreted model, if

(1) A set of storage variables $M=\left\{m_{i} \mid 1 \leq 1 \leq k\right\}$ is defined.

(2) For each process $P_{1}$, a set of input and output data sets is specified, where each data set is a subset of $M$,

Then the model is said to be semi-interpreted. Given a semi-interpreted model, if one defines

(1) The initial values of variables in $M$.

(2) A set of functions, one for each process $P_{i}$, which takes the values of the variables in the input data set, performs some transformations on these values, 
Page 3

and modifies the values of the output data set of $P_{1}$.

Then an interpreted model results. We are interested only in uninterpreted models since such a model is an abstraction of a real system that allows one to focus attention on the flow of control. Each of the nine classes of nets studied in the next section 18 an uninterpreted model. When we refer to an existing model (say $X$ ), we refer to the model that results when $X$ is stripped off all Interpretation.

\section{The Nine Classes of Nets}

In what follows, we will formally define the nine classes. We assume that the reader is thoroughly familiar with ordinary Petri nets. The definitions serve only to formalize intuitive notions, so that there is no ambiguity.

\subsection{Extended Petri Net Model}

- Definition 2.1.1: The extended Petri net model is a directed graph defined as a six-tuple $\left(T, P, A, M^{O}, F, f_{e}\right)$ where:

$T=\left\{t_{1} \mid 1 \leq 1 \leq n\right\}$ is a finite set of "transitions".

$P=\left\{p_{1} \mid 1 \leq 1 \leq m\right\}$ is a finite set of "places".

$A \subseteq\left\{\left(p_{1}, t_{j}\right) \cdot \mid p_{1} \varepsilon P, t_{j} \varepsilon T\right\} U\left\{\left(t_{1}, p_{j}\right) \mid t_{i} \in T, p_{j} \varepsilon P\right\} \rightarrow\left(p_{1}, t_{j}\right) \varepsilon: \dot{A} \Rightarrow\left(t_{j}, p_{i}\right)$

$\because \notin A$ and $\left(t_{1}, p_{f}\right) \in A \Rightarrow\left(p_{f}, t_{i}\right) \notin A, 1 s$ a finite set of directed. arcs.

Each arc connects a transition to a place or a place to a transition.

$\mathrm{M}^{\mathrm{O}}: \mathrm{P} \rightarrow \mathrm{N}:(\mathrm{N}$ is the set of non-negative integers) is a function that gives the number of "markers" in each place initially. $\mathrm{M}^{\circ}$ can be represented as $\left\{\left(p_{1}, n_{1}\right) \ldots\left(p_{m}, n_{m}\right)\right\}$ or where there is no ambiguity, as $\left(n_{1}, \ldots n_{m}\right)$. $F=\left\{F_{i} \mid 1 \leq i \leq p\right\}$ is a set of final markings where $F_{i}: P \rightarrow N U\{d\}$. (d denotes "don't care")

$\mathrm{f}_{\mathrm{e}}: \mathrm{A} \rightarrow$ True, False $\}$ Is a predicate on the arcs.

$T, P$ form the nodes of the graph. The transitions represent processes and the places, conditions. 
Definition 2.1.2: $I_{1}=\left\{j \mid\left(p_{j}, t_{1}\right) \varepsilon A\right\}$ is the set of indices of the input places of $t_{1}$.

Definition 2.1.3: $o_{1}=\left\{j \mid\left(t_{1}, p_{j}\right) \varepsilon A\right\}$ is the set of Indices of the output places of $t_{1}$.

Definition 2.1.4: $I_{1}^{\prime}=\left\{j \mid\left(p_{j}, t_{i}\right) \in A\right\}$ and $f_{e}\left(\left(p_{j}, t_{i}\right)\right)=$ True denotes a subset of the input places of $t_{1}$ for which $f_{e}$ is true.

Definition 2.1.5: $I_{i}^{\prime \prime}=\left\{j \mid\left(p_{j}, t_{i}\right) \varepsilon A\right.$ and $f\left(\left(p_{j}, t_{1}\right)\right)=$ False $\}$ denotes a subset. of the input places of $t_{i}$ for which $f_{e}$ is false.

Definition 2.1.6: $\mathrm{P}_{1}=$ number of markers in $\mathrm{P}_{i}$ at any instant.

Simulation Rules:

Transitions represent events or processes. The firfng of a transition represents the occurrence of an event. Whenever a transition $t_{i}$ desires to fire, 1t checks 1 ts input places and is enabled $1 f$ and only if $P_{j}>0$, for all $j$ $\varepsilon I_{1}^{\prime}$ and $P_{j}=0$, for all $j \varepsilon \cdot I_{1}^{\prime \prime}$. At this stage, a marker is removed from each $p_{j}, f \in I_{i}^{\prime}$ and the transition $t_{i}$ starts firing. At some later, finite, though a priori unknown and unbounded time, it completes firing and places a marker in each output place. Firing of a transition thus causes a change of state of the net and other transitions may be enabled or disabled as a result of this firing. In the actual graph, the number of markers in any place is shown by a corresponding number of "dots". The movement of markers cleariy shows which conditions cause a transition to fire and also which conditions come into being at the completion of firing.

The notion of conflict is important: During a simulation, two transitions are said to be in conflict if enabling of one prevents the enabling of the other and viá versa. In such a case the decision as to which one will be enabled is completely arbitrary. 
Page 5

Describing the Coordination Represented by a Net in this Model:

There are two ways of doing this. For each of these methods we require the notion of "trans-labelling".

Definftion 2.1.7: A trans-1abelling $I$ of a net $N=\left(T, P, A, M^{\circ}, F, f\right)$ is a mapping from $T^{\prime}$ onto $E$ where

$T^{\prime}=\left\{l_{1}, \ldots, \ell_{a}\right\} \subseteq$ I' is a set of transitions.

$E=\left\{E_{1}, E_{2}, \ldots E_{k}\right\}, k \leq a$ is a set of event or process names.

Thus, the same event or process name may be attached to different transitions and transitions in $T-T^{\prime}$ have no assoclated names. This is Indicated by $I(t)=$ for $t \varepsilon T-T^{\prime}$. It should be clear that the same net may represent different coordinations depending on its trans-labelling. The two ways of specifyling the coordination represented by a net are:

(1) By defining $T, P, A, M^{\circ}, F, f$ and giving a translabeling.

(2) By considering the "process sequences" produced by the net:

Definition 2.1.8: A marking $M$ is covered by some $F_{i}, 1 \leq 1 \leq p$ if $M(P)=$ $F_{1}(p)$, for all $p \ni F_{1}(p) \varepsilon N$.

Definftion 2.1.9: $\bar{t}=l_{1} ; \ell_{2}, \ldots \ell_{n} \varepsilon T *$ is said to be produced by a net $\mathrm{N}=\left(\mathrm{T}, \mathrm{P}, \mathrm{A}, \mathrm{M}^{\circ}, \mathrm{F}, \mathrm{f}\right)$ during a simulation if $\exists$ a sequence of markings $\mathrm{M}^{\circ}, \mathrm{M}^{1}$; $\ldots, M^{n} \Rightarrow \ell_{1}$ can be enabled $1 n M^{1-1}$ and firing of $\ell_{1}$ in $M^{1-1}$ results in $M^{1}$, $1 \leq 1 \leq n$. In addition, if $M^{n}$ is covered by some $F_{1} \varepsilon F, \bar{t}$ is sald to be a simulation sequence. of $\mathrm{N}$ :

Definition 2.1.10: The set of all simulation sequences of $M$ is called the simulation set of $\mathrm{N}$ or $\operatorname{SIMSET}_{\mathrm{N}}$.

Definition 2.1.11: Given a trans-labeling $I\left(T^{\prime}, E\right)$ of a net $N=(T, P, A$, $\left.M^{0}, F, f_{e}\right)$, for each simulation sequence $\bar{t}=\ell_{1}, \ell_{2}, \ldots, l_{n}$ we define a reduced simulation sequence $\bar{t}^{\prime}$ where $\bar{t}^{\prime}$ is obtained from $\bar{t}$ be deleting transitions in $T-T^{\prime}:$ 
Definition 2.1.12: Given a trans-labelling $I\left(T^{\prime}, E\right)$ of a net $N=(T, P, A$, $\left.M^{\circ}, F, f_{e}\right)$, for each reduced simulation sequence $\bar{t},-\ell_{1}, \ldots, \ell_{a}$ we define a process sequence $I\left(\bar{t}^{\prime}\right)=E_{c_{1}}, E_{c_{2}}, \ldots E_{c_{m}}$ where $I\left(\ell_{i}\right)=E_{c_{1}}, 1 \leq 1 \leq a$. Defintion 2.1.13: The set of all process sequences of $N$ given $I\left(T^{\prime}, E\right)$ is called I $\left(\operatorname{SIMSET}_{N}\right)$.

The coordination represented by a given net $N$ and trans-labeling $I$ is completely specifled by I (SIMSET $\left.{ }_{N}\right) \stackrel{*}{*}$

Let us call the class of extended Petrl nets $\mathrm{TN}_{\mathrm{EXT}}$ : In the following four classes, the slmulation rules are identical with those of extended Petri nets and definitions 2.1.2 through 2.1.14 carry over without change."

\subsection{Ordinary Petr1 Net Mode1}

Definition 2.2.1: The ordinary Petri net model is directed graph defined as a six-tuple ( $T, P, A, M^{0}, F, f_{e}$ ) where $\left(T, P, A, M^{\circ}, F, f_{e}\right) \varepsilon \cdot T_{E X T}$ and in addition, $f_{e}(a)=T$, for all a $\varepsilon A$.

Let the class of ordinary Petrl nets be TN.

\subsection{Bounded Petr1 Net Model}

Definition 2.3.1: The bounded Petri net model is a directed graph defined as a six-tuple ( $\left.T, P, A, M^{\circ}, F, f_{e}\right)$ where $\left(T, P, A, M^{\circ}, F, f_{e}\right) \varepsilon$ TN and in addition for each net $N$ in this class $\exists$ an a priori known bound $R ;$

(1) $\mathrm{M}^{\mathrm{O}}: \mathrm{P} \rightarrow\{0,1, \ldots, \mathrm{R}\}$

(2) $F_{i} \in F \Rightarrow F_{1}: P \rightarrow\{0,1,2, \ldots R\} \cup\{d\}$

(d denotes "don't care")

(3) There 1s no sequence $\bar{t}=\ell_{1}, \ell_{2}, \ldots, \ell_{n}$ produced by $N=M^{n+1}(p)>R$ for any place $p$ in $N$.

Let the class of bounded Petri nets be $\mathrm{TN}_{\mathrm{BDD}}$

\subsection{Safe Petri Net Model}

Definition 2.4.1: The safe Petri net model is a directed graph defined as a six-tuple $\left(T, P, A, M^{\circ}, F, f_{e}\right)$ where $\left(T, P, A, M^{O}, F, f_{e}\right) \varepsilon T N_{B D D}$ and $R=1$. 
Let the class of safe Petri nets be $\mathrm{TN}_{\text {safe }}$.

\subsection{The Marked Graph Mode1}

Definition 2.5.1: The marked graph model is a directed graph defined as a six-tuple ( $\left.T, P, A, M^{0}, F, f_{e}\right)$ where $\left(T, P, A, M^{0}, F, f_{e}\right) \varepsilon$ TN and in addition:

(1) for all $p_{1} \varepsilon P,\left(t_{j}, p_{1}\right) \varepsilon A$ and $\left(t_{k}, p_{1}\right) \varepsilon A \Rightarrow j=k$. .

(2) for all $p_{1} \varepsilon P,\left(p_{1}, t_{j}\right) \varepsilon A$ and $\left(p_{1}, t_{k_{0}}\right) \varepsilon A=>j=k$.

Thus, each place has at most one input transition and one output transition. Such a net can have no conflicts. Let the class of marked graphs be TN ${ }_{\text {MARK }}$.

\subsection{The Complex Petr1 Net Mode1}

Definition 2.6.1: The complex Petr1 net model is a directed graph defined as a slx-tuple $\left(T, P, A, M^{\circ}, F, f_{x}\right)$ where $T, P, A, M^{\circ}, F$ are the same as in definition 2.1 .1 and $\mathrm{f}_{\mathrm{x}}: \mathrm{A}+\mathrm{N}$.

Let $I_{i}, O_{1}$ and $P_{1}$ be as in definitions $2.1 .2,2.1 .3$, and 2.1 .6 respectively. Simulation Rules:

Whenever a transition $t_{1}$ desires to fire, it checks its input places. If $P_{j} \geq f_{x}\left(\left(p_{j}, t_{1}\right)\right)$ for all $f \varepsilon I_{1}$, then the transition is enabled. At this stage it removes $f_{x}\left(\left(p_{f}, t_{1}\right)\right)$ markers from each input place $p_{j} \cdot$ On completion of firing, $t_{1}$ places $f_{x}\left(\left(t_{1}, p_{f}\right)\right)$ markers in each output place $p_{f} \cdot$

Definftions 2.1.7 through 2.1.14 carry over unchanged. Let the class of complex Petri nets be TN PLEX:

\subsection{The OR Input Petri Net Mode1}

Definition 2.7.1: The OR input Petri net model is a directed graph defined as a six-tuple $\left(T, P, A, M^{\circ}, F, f_{X O R I}\right)$ where $T, P, A, M^{\circ}, F$ are the same as in definition 2.1.1, and $\mathrm{f}_{\mathrm{XORI}}: \mathrm{A}_{1} \rightarrow(+, *)$, where $\mathrm{A}_{1} \subset \mathrm{A}$ and consists of all arcs of the form $\left(p_{j}, t_{i}\right)$.

Let $I_{i}, O_{1}$ and $P_{1}$ be the same as in definitions $2.1 .2,2.1 .3$, and 2.1 .6 respectively.

Definition 2.7.2: $I_{1}^{*}=\left\{j \mid j \varepsilon I_{i}\right.$ and $\left.f_{X O R I}\left(\left(p_{f}, t_{i}\right)\right)=*\right\}$. 
Definition 2.7.3: $I_{1}^{+}$is the set difference of the sets $I_{1}$ and $I_{i}^{*}$. Simulation Rules:

Whenever a transition $t_{1}$ desires to fire it checks its input places. If $P_{j}>0$ for all $j \in I_{i}^{*}$ and $P_{k}>0$ for at least one $k \varepsilon I_{1}^{+}$, the transition is enabled. At this stage it removes one marker from each $P_{j} \ni j \varepsilon I_{i}^{*}$ and one marker from the $p l$ ace $p_{k}, k \in I_{i}^{+}$which satisfied the check. When $1 t$ completes firing, it places a marker in each output place.

Definftions 2.1.7 through 2.1.13 carry over unchanged. Let the class of nets be $\mathrm{TN}_{\mathrm{XORI}}$.

\subsection{The Exclusive OR Output Petri Net Mode1:}

Definition 2.8.1: The exclusive OR output Petri net model is a directed graph defined as a six-tuple ( $\left.T, P, A, M^{\circ}, F, f_{X O R O}\right)$ where $T, P, A, M^{\circ}, F$ are the same as in definition 2.1 .1 and $f_{X O R O}: A_{2} \rightarrow(*,+)$ where $A_{2} \subset A$ and contalns all arc of the form $\left(t_{i}, p_{f}\right)$.

Let $I_{1}, O_{1}$ and $P_{1}$ be the same as in definitions $2.1 .2,2.1 .3$ and 2.1 .6 respectively.

Definition 2.8.2: $0_{1}^{*}=\left\{j \mid j \varepsilon 0_{1}\right.$ and $\left.f_{\text {XORO }}\left(t_{i}, P_{j}\right)=*\right\}$. Definition 2.8.3: $\quad 0_{1}^{+}$is the set difference of the sets $o_{1}$ and $o_{1}^{*}$. Simulation rules:

Whenever a transition desires to fire, it checks 1 ts input places and is enabled if $\mathrm{P}_{1}>0$, for all $i \varepsilon I_{1}$. At this stage it removes a marker from each input place and starts firing. On completion of firing it places a marker in each $p_{j} \rightarrow j \in O_{1}^{*}$ and places one marker arbitrarily in one of the places $\mathrm{p}_{\mathbf{k}} \varepsilon$ 咩:

Definitions 2.1.7-2.1.14 carry over unchanged. Let the class of nets be TN XORO $^{\circ}$ 2.9 The Place Restricted Petri Net Model

Definition 2.9.1: The place restricted Petri net model is a directed graph defined as a six tuple $\left(T, P, A, M^{\circ}, F, f_{\text {OUT }}\right)$ where $T, P, A, M^{\circ}, F$ are the 
same as 1 definftions 2.1 .1 and $f_{\text {OUT }}: P \rightarrow\{$ True, False .

Let $I_{1}, O_{1}$ and $P_{1}$ be the same as in definitions $2.1 .2,2.1 .3$ and 2.1 .6 .

Simulation rules:

When a transition desires to fire, it checks its input places and is enabled if $P_{1}>0$, for all $1 \varepsilon I_{i}$ : At this stage 1 t removes one marker from each input place. On completion of firing, it places a marker in each output place $\mu \rightarrow f_{\text {OUT }}(p)=$ True. If $t_{1}$ has an output place $p \rightarrow f_{\text {OUT }}(p)=$ False, then a marker is placed in $p$ if and only if $P=0$.

Definttions 2.1.7 through 2.1.13 carry over unchanged. Let the class of nets be ${ }^{\mathrm{TN}}$ OUT ${ }^{\circ}$ 
Page 10

2.10 Summary

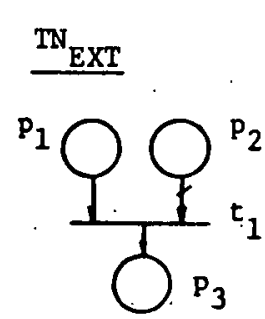

$f\left(p_{1}, t_{1}\right)=f\left(t_{1}, p_{3}\right)=T$

$f\left(p_{2}, t_{1}\right)=F$. This is indicated by a slash across $\left(p_{2}, t_{1}\right), t_{1}$ can be enabled if $P_{1}>0$ and $P_{2}=0$
TN

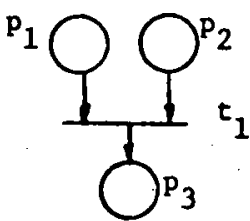

$f\left(\dot{p}_{1}, t_{1}\right)-f\left(p_{2}, t_{1}\right)=f\left(t_{1}\right.$, same as TN except there $\left.P_{3}\right)=T$. $t_{1}$ can be enabled is a bound. $R$, on the if $P_{1}>0$ and $P_{2}>0$.

TN number of markers in any place

\section{TN safe}

same as $\mathrm{TW}_{\mathrm{bdd}}$ except the bound is 1 bdd

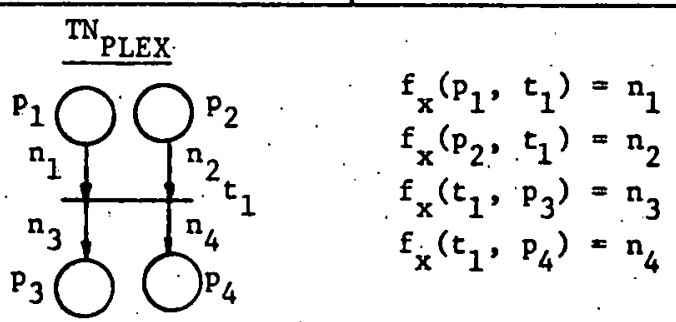

This is indicated by writing the values next to the arcs.

$t_{1}$ can be enabled if $P_{1} \geq n_{1}, P_{2} \geq n_{2}$. At this stage 1t removes $n_{1}$ markers from $p_{1}, n_{2}$ from $p_{2}$. On completion, it places $n_{3}$ markers in $p_{3}$ and $n_{4}$ in $p_{4}$

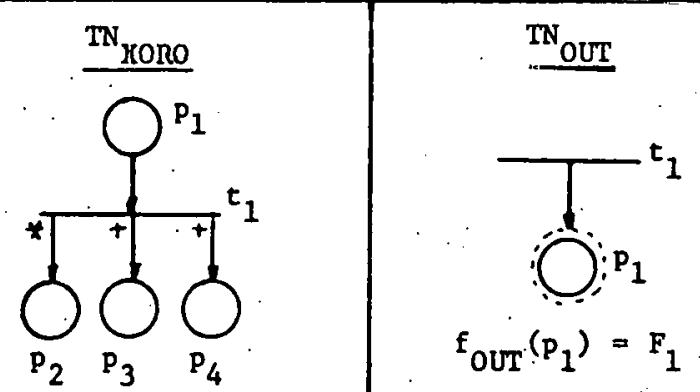

$\mathrm{f}_{\text {XORO }}\left(t_{1}, \mathrm{P}_{3}\right)=*, \mathrm{f}_{\mathrm{XORO}}$

A marker is placed in. $p_{1}$ if and only if $p_{1}=0$ : $t_{1}$ can be enabled if $P_{1}>0$ and $\left(P_{2}>0\right.$ or $\left.P_{3}>0\right) . t_{1}$ removes one marker from $p_{1}$ and one from elther $\mathrm{P}_{2}$ or $\mathrm{P}_{3}$ $\left(t_{1}, p_{3}\right)=f_{\text {XORO }}\left(t_{1}, p_{4}\right)=+$ When $t_{1}$ completes, it places a marker in $\mathrm{p}_{2}$ and one in $\mathrm{P}_{3}$ or $\mathrm{P}_{4}$. 


\subsection{The H1erarchy of Nets}

Definition 2.11.1: A net $N=\left(T, P, A, M^{0}, F, f\right)$ is well behaved (WB) if for any sequence $\ell_{1}, l_{2}, \ldots, l_{n}$ produced by the net either $M^{n}$ is covered by some $f_{1} \varepsilon F$ or there exists a way of reaching a marking which is covered by some $F_{1} \varepsilon F$.

Definttion 2.11.2: $\mathrm{TN}_{\mathrm{A}} \subseteq \mathrm{TN}_{\mathrm{B}}$ if and only if for every $\mathrm{N}_{\mathrm{A}} \varepsilon \mathrm{TN}_{\mathrm{A}}$ and trans-labelling $I_{A}, \exists$ a liet $N_{B} \varepsilon$ l'N $_{B}$ and trans-labelling $I_{B} \quad 3$

(1) $I_{A}\left(\operatorname{SIMSET}_{\mathrm{N}_{\mathrm{A}}}\right)=I_{\mathrm{B}}\left(\operatorname{SIMSET}_{\mathrm{NB}_{\mathrm{B}}}\right)$ and

(2) $\mathrm{N}_{A}$ is $\mathrm{WB} \Rightarrow \mathrm{N}_{B}$ 1s WB:

Definition 2.11.3: $\operatorname{TN}_{A}=\operatorname{TN}_{B}$ if and only if $\operatorname{TN}_{A} \subseteq \mathbb{T N}_{B:}$ and $\operatorname{TN}_{B} \subseteq T_{A}$. Defintition 2.11.4: $\mathrm{TN}_{A} \rightarrow \mathrm{TN}_{B}$ if and only; if $\mathrm{TN}_{B} \subseteq \mathrm{TN}_{\mathrm{A}}$ and $\mathrm{TN}_{\mathrm{A}} \nsubseteq \mathrm{TN}_{\mathrm{B}}$.

Intuitively, $\mathrm{TN}_{A}=\mathrm{TN}_{B}$ if and only if the two classes of nets can represent exactly the same class of coordinations. $\mathrm{TN}_{A} \rightarrow \mathrm{TN}_{B}$ if and only if $\mathrm{TN}_{A}$ is more powerful than $T_{B}$ with respect to its capability for representing coordinations. In what follows, we will establish the following hierarchy:

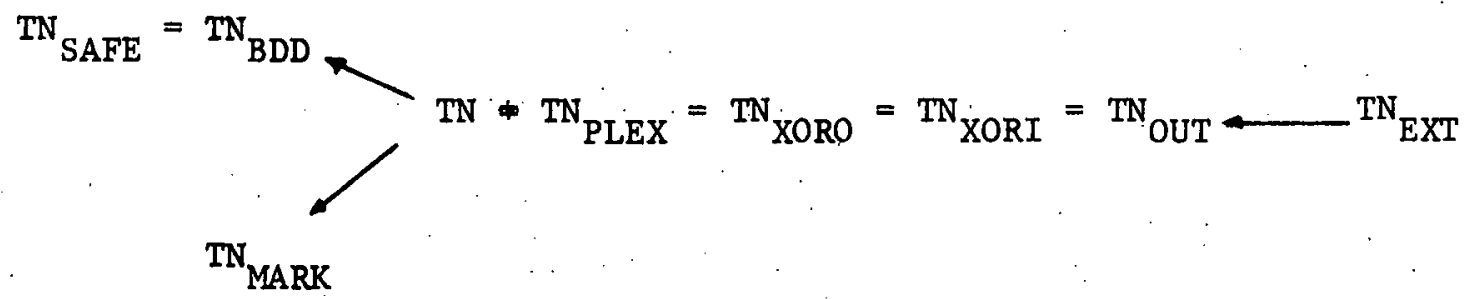

We will not go into detalled proofs in this paper, especially the proofs demonstrating equivalence. The reason is that these proofs are extremely tedious. One such detalled proof has been worked out [4] as an example. Some parts of this hlerarchy have already been established earlier and will not be discussed in this report. Theorem 2.11.1: $\operatorname{TN}_{\mathrm{EXT}} \rightarrow \mathrm{TN}$.

Established in [3]

Theorem 2.11.2: $\quad \mathrm{TN}^{-} \mathrm{TN}_{\text {OUT }}$. Established in $[2, \cdots 4]$. 
Page 12

Theorem 2.11:3: $\quad \mathrm{TN}_{\mathrm{SAFE}}=\mathrm{TN}_{\mathrm{BDD}}$

\section{Proof:}

Since $N \varepsilon \mathrm{TN}_{\mathrm{SAFE}} \Rightarrow \mathrm{N} \varepsilon \mathrm{TN}_{\mathrm{BDD}}, \mathrm{TN}_{\mathrm{SAFE}} \subseteq \mathrm{TN}_{\mathrm{BDD}}$. Thus we are only required to show that $\mathrm{TN}_{\mathrm{BDD}} \subseteq \mathrm{TN}_{\mathrm{SAFE}}$.

Let $\mathrm{N}_{1}=\left(\mathrm{T}_{1}, \mathrm{P}_{1}, \mathrm{~A}_{1}, \mathrm{M}_{1}^{0}, \mathrm{~F}_{1}, \mathrm{f}_{\mathrm{e}}^{1}\right) \varepsilon \mathrm{TN}_{\mathrm{BDD}}$ and let $\mathrm{I}_{1}$ be any translabelling of $N_{1}$. Let the bound be $R$. Then, the maximum number of possible markings of $\mathrm{N}_{1}$ 1s $(\mathrm{R}+1)^{\mathrm{m}}$ where $\mathrm{m}$ is the number of places. Each marking of $\mathrm{N}_{1}$ can be considered to be one system state of $N_{1}$ and can be written as an m-tuple. Let these markings be $Q_{1}, Q_{2}, \ldots Q_{(R+1)} m^{*} \quad\left(N_{1}\right.$ may never reach most of these markings but that is not of concern).

$$
\begin{aligned}
& \text { A net } \mathrm{N}_{2}=\left(\mathrm{T}_{2}, \mathrm{P}_{2}, \mathrm{~A}_{2}, \mathrm{M}_{2}^{0} ; \mathrm{F}_{2}, \mathrm{f}_{\mathrm{e}}^{2}\right) \text { is constructed as follows: } \\
& P_{2}=\left\{q_{1} \mid 1 \leq 1 \leq(R+1)^{m_{3}}\right\} \text { where each } q_{1} \text { corresponds to a unique marking } \\
& Q_{1} \text { of } \mathrm{N}_{1} \\
& T_{2}=\left\{t_{s}(1, j) \mid t_{s} \in T_{1} \text { can be enabled in } Q_{1} \text { and firing of } t_{s} \text { in } Q_{1}\right. \\
& \text { leads to } \left.Q_{j}\right\} \\
& A_{2}=\left\{\left(q_{1}, t_{s}(1, j)\right) \mid t_{s}(1, j) \varepsilon T_{2}\right\} \quad U \\
& \left\{\left(t_{s}(1, j), q_{j}\right) \mid t_{s}(1, j) \varepsilon T_{2}\right\} \\
& M_{2} \circ(q)=\left\{\begin{array}{l}
1 \text { if } q \text { corresponds to } M_{1} \circ \\
0 \text { otherwise }
\end{array}\right.
\end{aligned}
$$

Let $F^{\prime}$ be the set of $k$ markings of $N_{1} \ni Q_{r} \varepsilon . F^{\prime}$ if and only if $Q_{r}$ is covered by some $F_{j} \in F_{1}$, then

$F_{2}=\left\{M_{1}, M_{2}, \ldots, M_{k}\right\}$ where, for $1 \leq j \leq k$.

$M_{j}\left(q_{r}\right)=\left\{\begin{array}{l}1 \text { if } Q_{r} \varepsilon F^{\prime} \\ 0 \text { otherwise }\end{array}\right.$

$f_{e}^{2}(a)=$ True, for all a $\varepsilon A_{2}$

Thus, each final marking of $\mathrm{N}_{2}$ assigns one marker to $q_{i}$ if $Q_{1}$ is covered by a final marking of $\mathrm{N}_{2}$ and assigns zero markers to all other places of $\mathrm{N}_{2}$. At any stage in the oimulation of $N_{1}$ and $N_{2}$, let $N_{1}$ have marking $Q_{1}$ and in 
$\mathrm{N}_{2}$ let $\mathrm{q}_{1}$ be the only place containing a marker. If $t_{s}$ can be enabled in $\mathrm{N}_{1}$ at this stage and firing of $t_{s}$ results in $Q_{j}$, then $t_{s}(1, j)$ can be enabled in $\mathrm{N}_{2}$ (and if any $t_{s}(k, l)$ can be enabled then $k=1$ and $l=j$ ) and firing of $t_{s}$ $(i, j)$ results in a marking that assigns one marker to $q_{j}$ and zero markers to all other places. It is easy to see (by the definitions of $M_{2}^{0}$ and $F_{2}$ ). that $\ell_{1}, \ell_{2}, \ldots \ldots \ldots l_{k} \in \operatorname{SIMSET}_{N_{1}}$, with the corresponding sequence of markings $M_{1}^{1}=Q_{a_{1}}, 0 \leq 1 \leq k$ if and only if $l_{1}\left(a_{0}, a_{1}\right), l_{2}\left(a_{1}, a_{2}\right), \ldots$ $\ldots, l_{k}\left(a_{k-1}, a_{k}\right) \in \operatorname{SIMSET}_{N_{2}}$. If $I_{2}$ is now defined so that $I_{2}\left(t_{1}(x, y)\right)=$ $I_{1}\left(t_{i}\right)$ for all $t_{1}(x, y) \varepsilon T_{2}$ then $I_{1}\left(\operatorname{SIMSET}_{N_{1}}\right)=I_{2}\left(\operatorname{SIMSET}_{N_{2}}\right)$.

It is not difficult to see that $N_{2}$ is well behaved if $N_{1}$ is. Consider any sequence $\bar{t}=l_{I}\left(a_{0}, a_{1}\right), l_{2}\left(a_{1}, a_{2}\right), \ldots, l_{k}\left(a_{k-1}, a_{k}\right)$ produced by $N_{2}$. Then (see above) the sequence $\ell_{1}, l_{2}, \ldots, l_{k}$ can be produced by $N_{1}$, with $Q_{a_{1}}$, $0 \leq 1 \leq k$ being the corresponding markings. Since $N_{1}$ is well behaved, either (1) $Q_{a_{k}}$ is covered by some final marking of $N_{1}$ or (2) $\exists$ a sequence $\ell_{k+1}, \ldots, l_{p}$ $\rightarrow \ell_{1}, \ell_{2}, \ldots \ell_{k}, \ell_{k+1}, \ldots, \ell_{p}$ can be produced by $N$ (with a corresponding sequence of markings $\left.Q_{a_{1}}, 0 \leq 1 \leq p\right)$ such that $Q_{a_{p}}$ is covered by a final marking of $\mathrm{N}_{1}$

In case (1), the marking after $\bar{t}$ assign one marker to $q_{a_{k}}$ and zero markers to all other places., Clearly, this marking is covered by a final marking of $\mathrm{N}_{2}$ if $Q_{a_{k}}$ is covered by a final marking of $N_{1}$. In case (2), after $\bar{t}$, the sequence of firings $l_{k+1}\left(a_{k}, a_{k+1}\right), \ldots, l_{p}\left(a_{p-1}, a_{p}\right)$ can take place. The resulting marking assigns zero markers to all places except $\mathrm{q}_{\mathrm{a}_{\mathrm{p}}}$. This marking is also. covered by a final marking if $Q_{a_{p}}$ is.

Example 2.11.1: We will illustrate the above construction by means of an example. Let $N_{1}=\left(T_{1}, P_{1}, A_{1}, M_{1}^{o}, F_{1}, f_{e}^{1}\right) \varepsilon T_{B D D}$. The net is given in figure 2.11 .1 and $R=2$. 


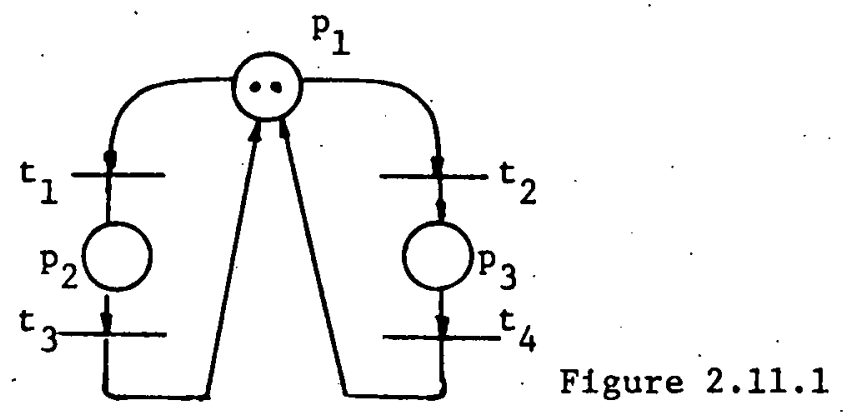

$M_{1}^{o}=\left\{\left(p_{1}, 2\right),\left(p_{2}, 0\right),\left(p_{3}, 0\right)\right\}$ or more briefly, $(2,0,0)$

$\mathrm{F}_{1}=\left\{\mathrm{M}_{1}^{\mathrm{o}}\right\}$

of the 27 markings, the only possible markings that $\mathrm{N}_{1}$ can attain are:

$Q_{1}=(2,0,0), Q_{2}=(1,1,0), Q_{3}=(0,2,0), Q_{4}=(1,0,1), Q_{5}=(0,0,2)$,

$Q_{6}=(0,1,1)$. Thus, the net $N_{2}$ to be constructed has 6 places $q_{1}, q_{2}, q_{3}$, $q_{4}, q_{5}$ and $q_{6}, F^{\prime}$ for this case is the set $\{(2,0,0)\}$. Thus, $M_{2}^{0}=\{(q, 1)$, $\left.\left(q_{2}, 0\right) ;\left(q_{3}, 0\right),\left(q_{4}, 0\right),\left(q_{5}, 0\right),\left(q_{6}, 0\right)\right\}$, or more brtefly, $(1,0,0,0,0,0)$. $F_{2}=\{(1,0,0,0,0,0)\}$. The net $N_{2}=\left(T_{2}, P_{2}, A_{2}, M_{2}^{0}, F_{2}, f_{e}^{2}\right)$ is given in f1gure 2.11.2.

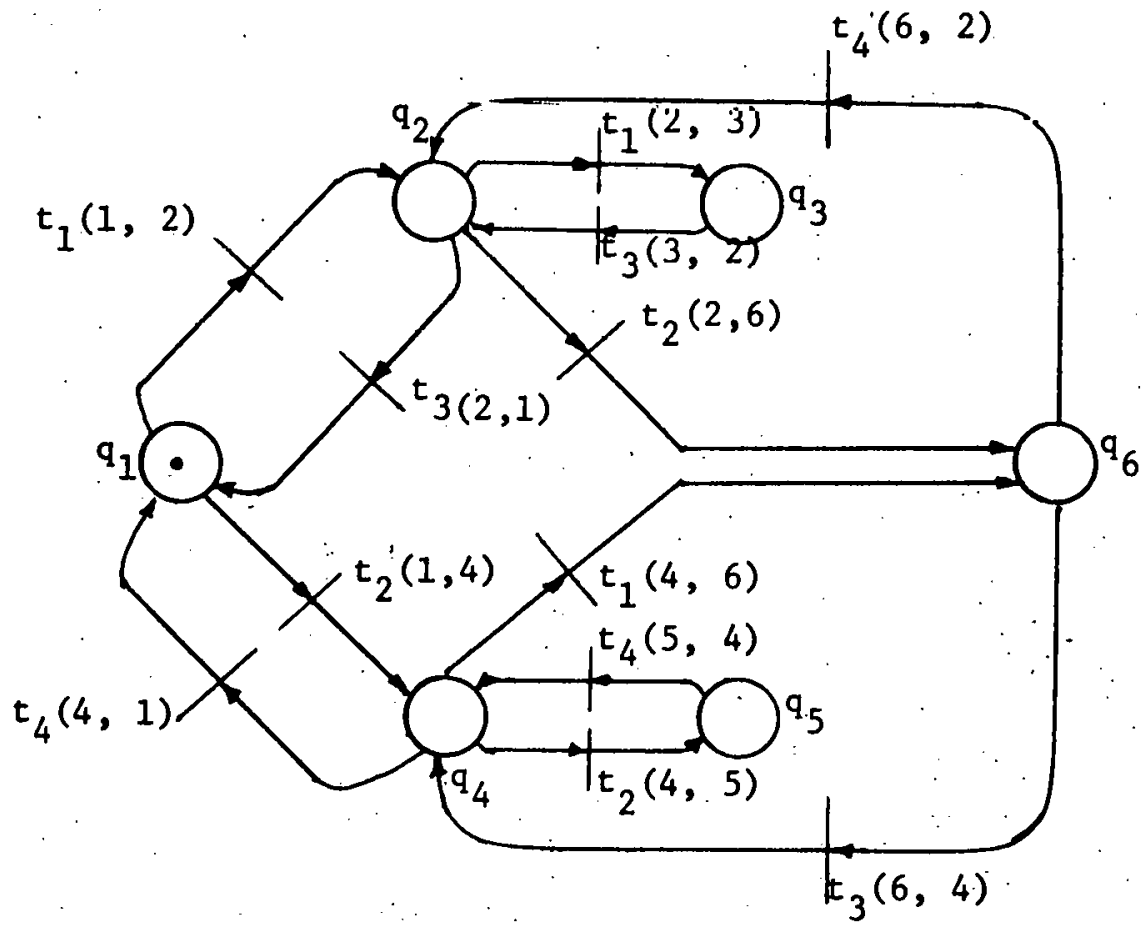

Figure 2.11.2 
Let $I_{1}$ be a trans- labelling of $N_{1} \rightarrow I_{1}\left(t_{1}\right)=P_{1}, I_{1}\left(t_{2}\right)=P_{2}, I_{1}\left(t_{3}\right)=$ $P_{3}$. Then define $I_{2} \rightarrow I_{2}\left(t_{1}(1, j)\right)=P_{1}, I_{2}\left(t_{2}(1, f)\right)=P_{2}, I_{2}\left(t_{3}(1, f)\right)=$ $\mathrm{P}_{3}$. Then, $\mathrm{I}_{1}\left(\operatorname{SIMSET}_{\mathrm{N}_{1}}\right)=\mathrm{I}_{2}\left(\operatorname{SIMSET}_{\mathrm{N}_{2}}\right)$ and both $\mathrm{N}_{1}$ and $\mathrm{N}_{2}$ are, well behaved. Theorem 2.11.4: TN $_{\text {MARK }}+$ TN

Proof:

Since $N \in T_{\text {MARK }} \Rightarrow N \varepsilon T N, T_{M A R K} \subseteq T N$. Thus, we only have to prove that $\mathrm{TN} \nsubseteq \mathrm{TN}_{\mathrm{MARK}} \cdot$ Let $\mathrm{N}=\left(\mathrm{T}, \mathrm{P}, \mathrm{A}, \mathrm{M}^{\circ}, \mathrm{F}, \mathrm{f}\right) \varepsilon \mathrm{TN}_{\mathrm{MARK}} \cdot$ In $\mathrm{N}_{1}$, each place. has at most one input transition and one output transition. We can thus talk of any transtition being either in the enabled (E) state or disabled (D) state at any stage in a simulation. If a transition is in an $E$ state, no other transition can affect this state. The only way the transition can possibly go into the $D$ state is by firfing (itself). Let $\ell^{n}=l_{1}, l_{2}, \ldots l_{n}$ and $d^{n}=$ $\mathrm{d}_{1}, \mathrm{~d}_{2}, \therefore \therefore, \mathrm{d}_{\mathrm{n}}$ be any strings produced by $\mathrm{N}$. Let $\mathrm{C}_{1} \square \mathrm{C}_{2}$ indicate the concatenation of two strings $C_{1}$ and $C_{2}$.

\section{Algorithm:}

Stage (i); Examine $l_{1}$ and $d_{1}$

1. If $l_{1}$ and $d_{1}$ already included in $c$ then start stage $1+1$.

2. If $l_{1}=d_{1}$ and $l_{1}$ not included in $c$, then $c+c \square \ell_{1}$; start stage $i+1$.

3. If $l_{1} \neq d_{1}$ and $l_{1}$ included in $c$, then $c+c \square d_{1}$; start state $1+1$.

4. If $\ell_{1} \neq d_{1}$ and $d_{1}$ included in $c$ then $c+c \square \ell_{1}$; start stage $1+1$.

5. If $l_{1} \neq d_{1}$ and neither $\ell_{1}$ nor $d_{1}$ included in $c$ then $c+c$ a $l_{1} \square d_{1} ; L$

Start at stage 1 with $C=$ null string. Continue until stage $n$. Let

$C^{\mathrm{n}}$ be the resultant string $C$.

Lemma 2.11.1: $\mathrm{C}^{\mathfrak{n}}$ can be produced by $\mathrm{N}$.

Proof: (by induction)

$\mathrm{n}=1 ; \ell^{\mathrm{n}}=\ell^{1}=\ell_{1}, \mathrm{~d}^{\mathrm{n}}=\mathrm{d}^{1}=\mathrm{d}_{1}$. Then $\ell_{1}$ and $\mathrm{d}_{1}$ are both in the $\mathrm{E}$ state in the initial marking. If $\ell_{1}=d_{1}$, the $c^{n}\left(=\ell_{1}\right)$ can be produced by $N$. If $\ell_{1} \neq \mathrm{d}_{1}$, then $\mathrm{c}^{1}=\ell_{1}, \mathrm{~d}_{1}$. In this case also $\mathrm{c}^{1}$ can be produced by $\mathrm{N}$ since $\ell_{1}$ cannot cause $d_{1}$ to go from an $E$ state to a $D$ state. 
Let $\ell^{n}=\ell_{1}, \ldots \ell_{n} ; d^{n}=d_{1}, \ldots d_{n}$ and assume $c^{n}$ can be produced by N. Let $\ell^{n+1}=l_{1}, \ldots l_{n}, l_{n+1} ; d^{n+1}=d_{1}, \ldots, d_{n}, d_{n+1}$. Applying the algorithm unt1l stage $n$, we get $\mathrm{C}^{n}$ as an intermediate value of $c$. By the inductive hypothesis, $\mathrm{C}^{\mathrm{n}}$ can be produced by $\mathrm{N}$.

1. $\ell_{n+1}$ and $d_{n+1}$ already included in $c^{n}$. Then $c^{n+1}=c^{n}$ and $c^{n+1}$ can be produced by $\mathrm{N}$.

2. $\ell_{n+1}=d_{n+1}$ and $\ell_{n+1}$ not included in $c^{n}$. Then $c^{n+1}=c^{n} D l_{n+1} \cdot l_{n+1}$ does not occur in $\ell^{n}$ efther. $l_{n+1}$ is in an $E$ state after $\ell^{n}$. Let $p_{1}, p_{2}, \ldots, p_{r}$ be the Input places of $\ell_{n+1}$. Without loss of generality out of these places, let $p_{1}, p_{2}, \ldots, p_{u}$ be the places that were empty in the initial marking. Let $t_{1}, \ldots t_{s}$ be the 1nput places of these transitions. Then, $t_{1}, \ldots t_{s}$ must occur in some order in $\ell^{n}$, since it is only by the firing of these transitions that $\ell_{n+1}$ can reach the $E$ state. Once enabled, $\ell_{n+1}$ cannot go to the $D$ state, since 1t does not occur in $f^{n}$. However, the algorithm guarantees that $t_{1}, \ldots t_{s}$ occur in $\mathrm{c}^{\mathrm{n}}$ (1n some order). F1ring of these transitions must enable $l_{n+1}$. Therefore $l_{n+1}$ w1ll be in the $E$ state after $c^{n}$. Therefore $C^{n} \ell_{n+1}=c^{n+1}$ can be produced by $N$.

The arguments for cases 3 and 4 of the algorithm are similar to those for case 2 . In case 5 , the argument can be applied twlce to prove that after $c^{\mathrm{n}}$, both $\ell_{n+1}$ and $d_{n+1}$ are 1 the $E$ state. Since $\ell_{n+1} \neq d_{n+1}, d_{n+1}$ will be in the E state after $c^{n} \ell_{n+1}$ and hence $c^{n+1}=c_{n} \ell_{n+1} d_{n+1}$ can be produced by $N$. This completes the proof of lemma 2.11.1.

Let $\ell^{n}=\ell_{1}, \ell_{2}, \ldots, l_{n}$ and $d^{m}=d_{1}, \ldots, d_{m}$ be two strings produced by $\mathrm{N}, \mathrm{n}<\mathrm{m}$. It can be shown that if $l^{\mathrm{m}}=\ell_{1}, \ldots, l_{\mathrm{n}} l_{\mathrm{n}+1}, \ldots, l_{\mathrm{m}}$, where $\ell_{n+1}, \ldots, \ell_{m}$ are "null" and the algorithm applied to $\ell^{m}$ and $d^{m}$ to yield $c^{m}$, then $\mathrm{c}^{\mathrm{m}}$ can . by produced by $\mathrm{N}$.

Consider $\quad N_{1}=\left(T_{1}, P_{1}, A_{1}, M_{1}^{0}, F_{1}, f_{e}^{1}\right) \varepsilon T_{S A F E}$ given in figure 2.11.3. 


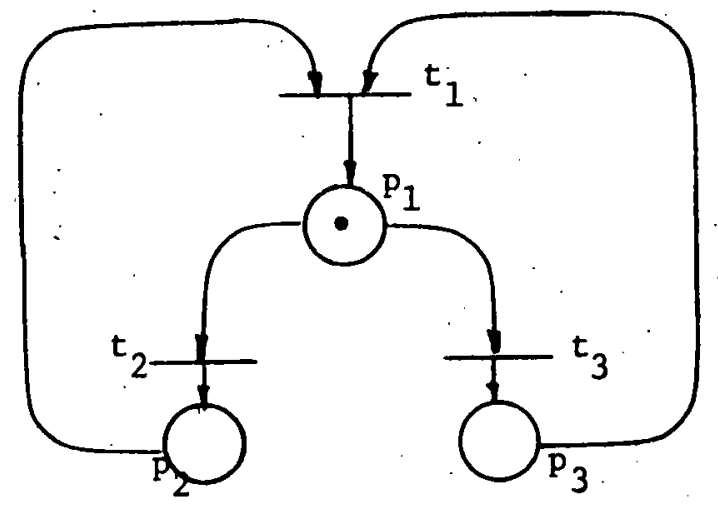

F1gure 2.11:3

$M_{1}^{0}=\left\{\left(p_{1}, 1\right),\left(p_{2}, 0\right),\left(p_{3}, 0\right)\right\}$

$\mathrm{F}_{1}=\left\{\mathrm{M}_{1}^{\mathrm{o}}\right\}$

Let $I_{1}$ be a trans-1abelling of $N_{1} \ni I_{1}\left(t_{1}\right)=P_{1}, I_{1}\left(t_{2}\right)=P_{2}, I_{1}\left(t_{3}\right)=P_{3}$. Any sequence belonging to $I_{1}\left(\mathrm{SIMSET}_{\mathrm{N}_{1}}\right)$ has the following characteristics. The sequence starts with $P_{2}$ or $P_{3}$ and between any two occurrences of $P_{2}$ and $P_{3}$ there must be at least one occurrence of $P_{1}$. Thus, for example, ${ }_{2}{ }_{2}{ }_{1} P_{3} P_{1} \varepsilon$ $I_{1}\left(\operatorname{SIMSET}_{N_{1}}\right.$ ) and $\mathrm{P}_{3} \mathrm{P}_{1} \mathrm{P}_{2} \mathrm{P}_{1} \varepsilon \mathrm{I}_{1}$ (SIMSET $\mathbb{N}_{1}$ ). Also, $\mathrm{N}_{1}$ is well behaved. Let $\mathrm{N}_{2}=\left(\mathrm{T}_{2}, \mathrm{P}_{2}, \mathrm{~A}_{2}, \mathrm{M}_{2}^{\circ}, \mathrm{F}_{2}, \mathrm{f}_{\mathbf{e}}^{2}\right) \in \mathrm{TN}_{\mathrm{MARK}}$ and let $\mathrm{I}_{2}$ be a trans-labelling of $\mathrm{N}_{2} \rightarrow$ $I_{2}\left(\operatorname{SIMSET}_{N_{2}}\right)=I_{1}\left(\right.$ SIMSET $\left._{N_{1}}\right)$ and $N_{2}$ is well behaved. Then, ${ }_{2}{ }_{2} P_{1} P_{3} P_{1} \varepsilon I_{2}$ $\left(\operatorname{SIMSET}_{\mathrm{N}_{2}}\right)$ and $\mathrm{P}_{3} \mathrm{P}_{1} \mathrm{P}_{2} \mathrm{P}_{1} \in I_{2}\left(\operatorname{SIMSET}_{\mathrm{N}_{2}}\right)$. Let $\ell_{1}, l_{2}, \ldots, l_{\mathrm{p}}$ be the simulation sequence from which $P_{2} P_{1} P_{3} P_{1}$ was obtained and let $d_{1}, d_{2}, \ldots, d_{q}$ be the sequence from which $P_{3} P_{1} P_{2} P_{1}$ was obtained. Let $I_{2}\left(l_{n}\right)=P_{2}$ for some $n<p$ and let $I_{2}$ $\left(l_{i}\right)=\emptyset ; 1 \leq 1<n$. Let $I_{2}\left(d_{m}\right)-P_{3}$ for some $m<q$ and let $I_{2}\left(d_{1}\right)=$ $\emptyset, 1 \leq 1<\mathrm{m}$. Both these sequences are produced by $\mathrm{N}_{2}$. Without loss of generality, let $\mathrm{n} \leq \mathrm{m}$. Applying the algorithm to $\ell^{\mathrm{n}}$ and $\mathrm{d}^{\mathrm{m}}$ yields $\mathrm{C}^{\mathrm{m}}$, where all distinct transitions in $\ell^{n}$ and $d^{m}$ appear once and only once, and $c^{m}$ can be produced by $\mathrm{N}_{2}$. Since $\mathrm{N}_{2}$ is well behaved by assumption, either the marking after $C^{m}$ is covered by a final marking or 3 a sequence $c_{m+1}, \ldots, c_{n} \rightarrow$ the marking after $C^{m} C_{m+1}, \ldots, C_{n}$ is covered by a final marking of $N_{2}$. Thus either one of the two sequences $\mathrm{P}_{2} \mathrm{P}_{3}$ and $\mathrm{P}_{3} \mathrm{P}_{2}$, belongs to $\mathrm{I}_{2}\left(\operatorname{SIMSET}_{\mathrm{N}_{2}}\right.$ ) or one of the two sequence $\mathrm{P}_{2} \mathrm{P}_{3}$ and $\mathrm{P}_{3} \mathrm{P}_{2}$ is a prefix of a process sequence belonging to $\mathrm{I}_{2}$ 
$\left(\operatorname{SIMSET}_{\mathrm{N}_{2}}\right)$. In each case, $\mathrm{I}_{1}\left(\operatorname{SIMSET}_{\mathrm{N}_{1}}\right) \neq \mathrm{I}_{2}\left(\operatorname{SIMSET}_{\mathrm{N}_{2}}\right) \cdot \mathrm{N}_{1} \in \mathrm{TN}_{\mathrm{SAFE}}$, therefore $\mathrm{N}_{1} \in \mathrm{TN}$. Thus TN $\nsubseteq \mathrm{TN}_{\text {MARK }}$ and $\mathrm{TN} \rightarrow \mathrm{TN}_{\text {MARK }}$. This completes the proof of theorem 2.11.4.

Theorem 2.11.5: $\mathrm{TN}_{\mathrm{SAFE}}+\mathrm{TN}$.

Proof:

A trans-labelled net $N=\left(T, P, A, M^{O}, F, f\right)$ can be considered to be a language generator, where I(SIMSET ${ }_{N}$ ) is the language generated. We w11l show first that the class of languages generated by trans-labelled, safe Petri nets is the class of regular languages.

1. Let $M=\left(K, \Sigma, \delta, q_{0}, F\right)$ be any finite state machine where

$K=\left\{q_{1} \mid 0 \leq 1 \leq n\right\}$ is a finite set of states

$\Sigma=\left\{a_{1} \mid 1 \leq 1 \leq \mathrm{m}\right\}$ is a finite alphabet

$\delta: \mathrm{KX} \Sigma \rightarrow \mathrm{K}$

$q_{0}:$ initial state

$F=\left\{b_{1}, b_{2}, \ldots \ldots b_{r}\right\} \subseteq K$, is the set of final states.

If $\delta\left(q_{1}, a_{j}\right)=q_{k}$, the machine $M$ In state $q_{i}$ scanning $a_{j}$ goes to state $q_{k} \cdot \bar{a} \varepsilon \Sigma^{*}$ Is a string accepted by, $M$ if $\bar{a}$ causes $M$ to go into a state' $q$ starting from $q_{0}$ $q \varepsilon F$. The set of all strings accepted by $M$ defines the language $L_{M}$ accepted by $M$. We will construct a trans-labelied, safe Petri net $N=\left(T, P, A, M^{\circ}, F, f_{e}\right)$ which generates the same language that $M$ accepts. $N$ is defined as follows:

$$
\begin{aligned}
T= & \left\{l_{1} \mid 1 \leq 1 \leq m\right\} U\left\{t_{i_{j}} \mid 0 \leq 1 \leq n, 1 \leq j \leq m\right\} \\
P= & \left\{q_{1} \mid 0 \leq 1 \leq n\right\} U\left\{p_{i_{j}} \mid 0 \leq 1 \leq n, 1 \leq j \leq m\right\} \\
A= & \left\{\left(q_{1}, l_{j}\right) \mid 0 \leq 1 \leq n, 1 \leq j \leq m\right\} U \\
& \left\{\left(p_{1_{j}}, t_{1_{j}}\right) \mid 0 \leq 1 \leq n, 1 \leq j \leq m\right\} U \\
& \left\{\left(l_{j}, p_{i_{j}}\right) \mid 0 \leq 1 \leq n, 1 \leq j \leq m\right\} U \\
& \left\{\left(t_{1}, q_{k}\right) \mid \delta\left(q_{1}, a_{j}\right)=q_{k}\right\} \\
M^{O}= & \left\{\left(q_{0}, 1\right),\left(q_{1}, 0\right), \ldots \ldots,\left(q_{n} ; 0\right)\right\}\left\{\left(p_{i_{j}}, 0\right) \mid 0 \leq 1 \leq n, 1 \leq j \leq m\right\} . \\
F= & \left\{M_{1} \mid 1 \leq 1 \leq r\right\} \text { where }
\end{aligned}
$$


Page 19

$F_{1}(x)=\left\{\begin{array}{l}1 \text { if } x \text { is } b_{1} . \\ 0 \text { otherwise }\end{array}\right.$
$\mathrm{F}_{e}(a)=$ True, for all a $\varepsilon A$.

The trans-labelling I is defined as follows:

$I\left(l_{1}\right)=a_{j}, 1 \leq j \leq m, 0 \leq 1 \leq n$

$I\left(t_{i_{j}}\right)=\emptyset, 1 \leq j \leq m, 0 \leq 1 \leq n$.

$N$ is safe and in fact at any stage of a simulation, $N$ has exactly one marker. Clearly, $I\left(\right.$ SIMSET $\left._{N}\right)=L_{M}$ and an example w111 make this clear.

Example 2.11.2: Figure 2.11.4 gives the state diagram of a finite state automaton M. The nodes represent states. An arc labelled $a_{k}$ exists between $q_{1}$ and $q_{j}$ If and only if $\delta\left(q_{1}, a_{k}\right)-q_{j}$. The final states are circled. $q_{0}$ is the initial state.

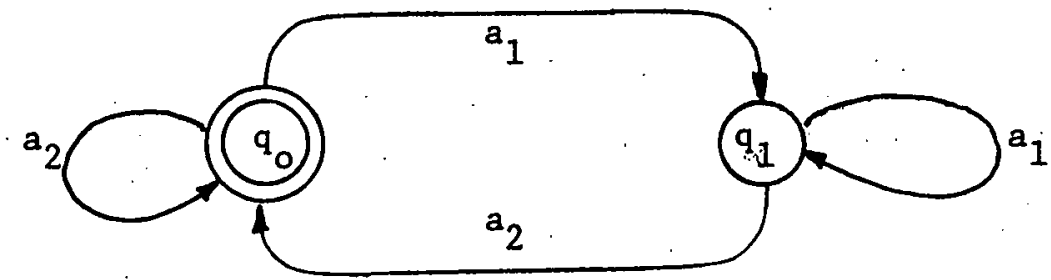

F1gure 2.11 .4

A trans-labelled safe Petri net is given in figure 2.11.5.

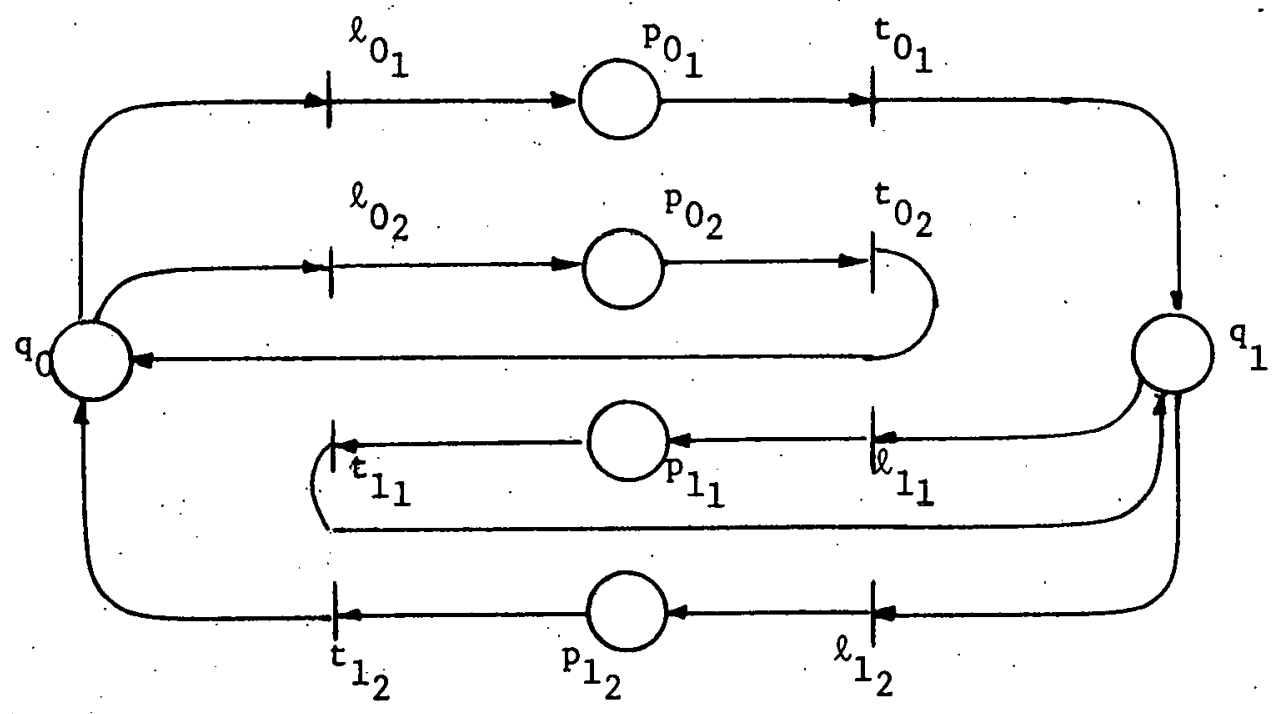

Figure 2.11.5 
$M^{0}=\left\{\left(q_{0}, 1\right),\left(q_{1}, 0\right)\right\}$

$F=\left\{\mathrm{M}^{\mathrm{O}}\right\}$

$I\left(l_{0_{1}}\right)=I\left(l_{1}\right)=\dot{a}_{1}$

$I\left(l_{0_{2}}\right)=I\left(l_{1_{2}}\right)=a_{2}$

$I\left(t_{i_{j}}\right)=\emptyset, 1=0,1, j=1,2$.

The sequence same place cannot be an input and output place of the same transition. The reader can easily verify that the process sequences of $\mathrm{N}$ are exactly the sentences recognized by $M$.

2. Let $N=\left(T, P, A, M^{0}, F, f_{e}\right)$ be any safe Petri net with trans-labeling $I$.

In the proof 2.11.3, given a trans-labelled net $\mathrm{N}_{1} \varepsilon \mathrm{TN}_{\mathrm{BDD}}$, a net $\mathrm{N}_{2} \varepsilon \mathrm{TN}_{\mathrm{SAFE}}$ was constructed $\exists$

a) $\mathrm{N}_{2}$ contained exactly one marker at any stage in a simulation.

b) $I_{1}\left(\operatorname{SIMSET}_{\mathrm{N}_{1}}\right)=I_{2}\left(\operatorname{SIMSET}_{\mathrm{N}_{2}}\right)$

c) $\mathrm{N}_{1} 18$ well behaved $\Rightarrow \mathrm{N}_{2} 18$ well behaved

d) Each transition has exactly one input and one vutput placc.

Since $N \in \mathrm{TN}_{\mathrm{BDD}}$, the construction yields a. similar net $\mathrm{N}_{2} \cdot \mathrm{I}_{2}$ can be redefined as follows: $I_{2}\left(t_{S}(1, j)\right)=I_{1}\left(t_{S}\right)$ if $I_{1}\left(t_{S}\right) \neq \emptyset$ and $I_{2}\left(t_{S}(1, j)\right)=P R$ otherwise where $P R \neq I_{1}\left(t_{S}\right)$ for all s. Clearly, I(SIMSET $)=I_{2}\left(\right.$ SIMSET $\left._{N_{2}}\right)$ if all occurrences of PR are deleted from each process sequence belonging to $\mathrm{I}_{2}\left(\right.$ SIMSET $\left._{2}\right)$. A state dlagram can be obtained from $\mathrm{N}_{2}$ by removing the transitions and labelling each arc $\left(q_{1}, q_{j}\right)$ by $t$ where $t$ was the transition between $q_{1}$ and $q_{j}$. The $q_{f}^{\prime} s$ form the states of the state diagram : The inftial state of this state diagram is the place that inftlally contained a marker in $\mathrm{N}_{2}$. The final states are those states assigned a marker by the final markings of $\mathrm{N}_{2} \cdot$ The finite state machine so obtained recognizes a language $\mathrm{L}_{M^{*}}$ Clearly, $L_{M}=I\left(\operatorname{SIMSET}_{N}\right)$ if all occurrences of $P R$ in each sentence of $L_{M}$ are 
deleted. Since regular languages are closed under homomorphisms, $\exists$ a regular language $L_{M}$ ' (obtained from $L_{M}$ by deleting all occurrences of $P R$ ) $\rightarrow L_{M}=$ I (SIMSET S $_{\mathrm{N}}$.

1 and 2 above prove that the class of languages generated by translabelied, safe Petri nets is the class of regular languages.

Cons1der the net $\mathrm{N}_{1} \varepsilon \mathrm{TN}$ given in Figure 2.11.6.

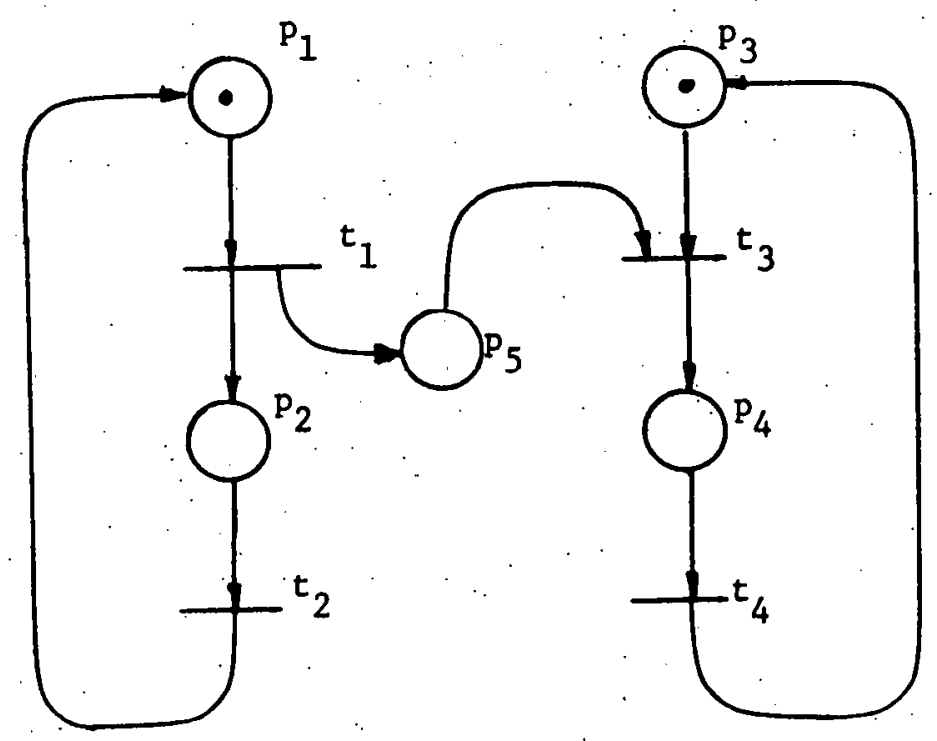

F1gure 2.11.6

$M^{0}=\left\{\left(p_{1}, 1\right),\left(p_{2}, 0\right),\left(p_{3}, 1\right), \cdots\left(p_{4}, 0\right),\left(p_{5}, 0\right)\right\}$

$F=\left\{M^{0}\right\}$

Let $I_{1}$ be a translabelling of $N_{1} \rightarrow I_{1}\left(t_{1}\right)="\left(", I_{1}\left(t_{3}\right)="\right) ", f_{1}\left(t_{2}\right)=$ $I_{1}\left(t_{4}\right)=\emptyset$. Then, $I_{1}\left(S_{M S E T}\right)$ is the set of well formed parenthesis expressions. This language 18 not regular: Therefore, no trans-labelled, safe Petri net can generate this language.. This concludes the proof of Theorem 2.11 .5

Theorem 2.11.6: TN $\div \mathrm{TN}_{\text {XORO }}$

\section{Proof:}

$N \in T N \Rightarrow N \varepsilon T_{X O R O}$, therefore $T_{N} \subseteq T_{X O R O} \cdot$. We are only required to show 
that $\mathrm{TN}_{\mathrm{XORO}} \subseteq \mathrm{TN}$. Consider any $\mathrm{N}_{1}=\left(\mathrm{T}_{1}, \mathrm{P}_{1}, \mathrm{~A}_{1}, \mathrm{M}_{1}^{\mathrm{O}}, \mathrm{F}_{1}, \mathrm{f}_{\mathrm{XORO}}^{1}\right) \mathrm{TNN}_{\mathrm{XORO}}$. Let $t_{1}$ be any transition in this net, $\ni I_{1}=\{1,2, \ldots, s\}, 0_{1}^{*}=\{s+1, \ldots, t\}$, $0_{1}^{+}=\{t+1, \ldots, t+r\}$. Thus, local to $t_{1}$, the net is as shown in Figure 2.11 .7 .

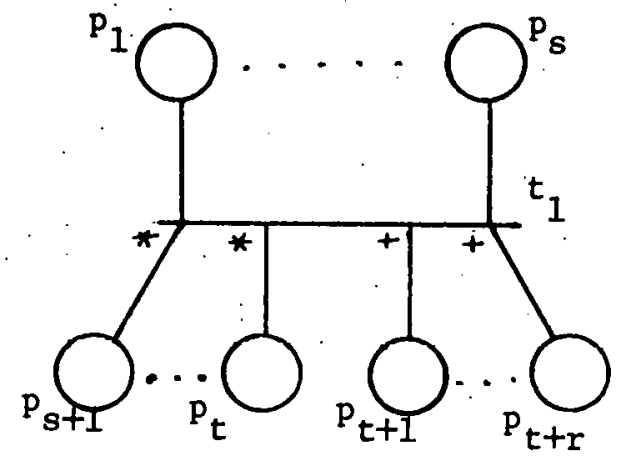

F1gure 2.11.7

Let the net $\mathrm{N}_{2}=\left(\mathrm{T}_{2}, \mathrm{P}_{2}, \mathrm{~A}_{2}, \mathrm{M}_{2}^{\circ}, \mathrm{F}_{2}, \mathrm{f}_{\mathrm{XORO}}^{2}\right)$ be obtained by replacing $\mathrm{t}_{1}$ by the net in figure 2.11 .8 .

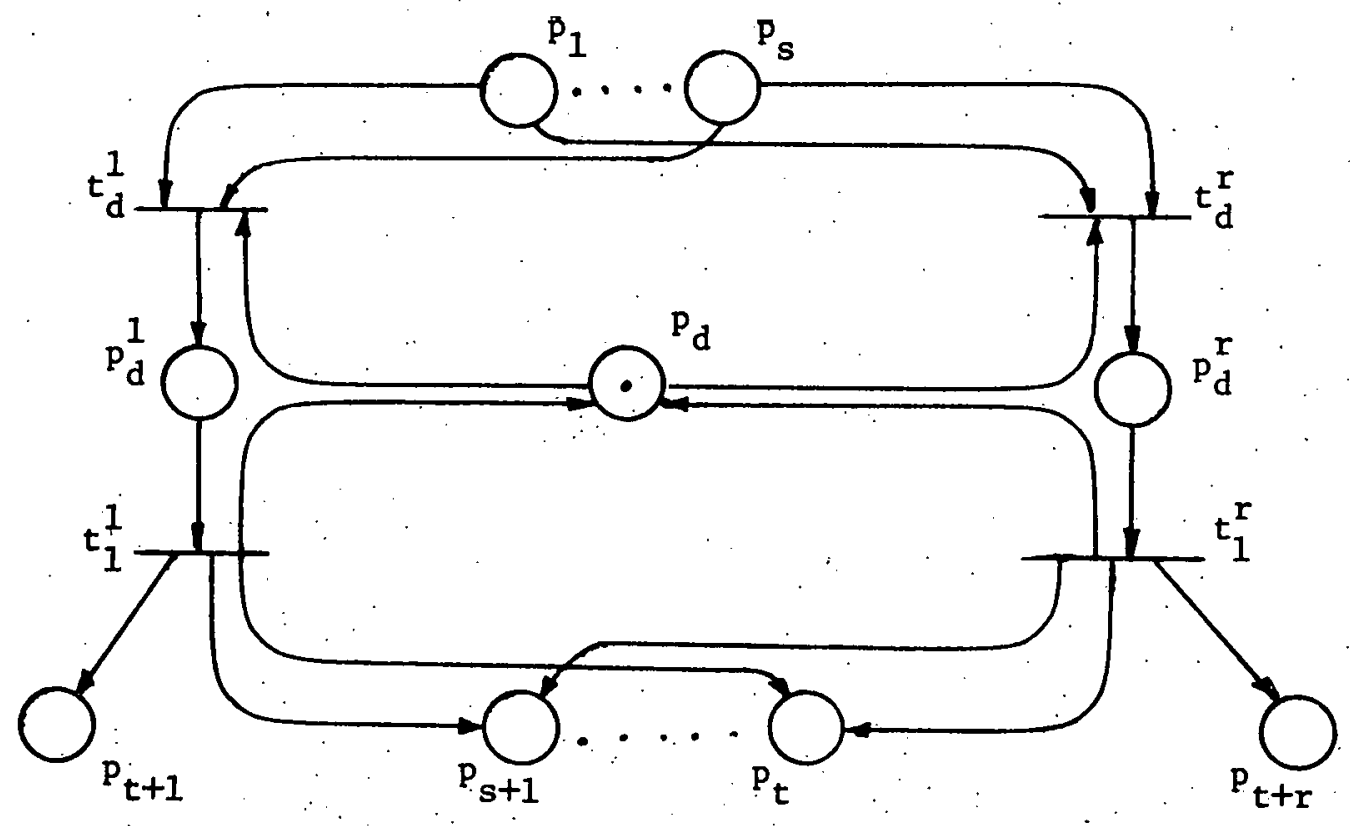

Figure 2.11.8 
If $M_{2}$ is a marking of $N_{2}$ and $M_{1}$ of $N_{1}$ then we say $M_{2}\left(P_{1} \cap P_{2}\right)=M_{1}\left(P_{1}\right)$ if $M_{2}\left(p_{1}\right)=M_{1}\left(p_{1}\right)$ for all $p_{1} \in P_{1}$ : Similarly, if $I_{2}$ is a trans-labelling of $N_{2}$ and $I_{1}$ of $N_{1}$ then $I_{2}\left(T_{1} \cap T_{2}\right)=I_{1}\left(T_{1}\right)$ if $I_{2}\left(t_{1}\right)=I_{1}\left(t_{1}\right)$ for all $t_{1} \varepsilon T_{1}$.

The construction of $\mathrm{N}_{2}$ defines $\mathrm{T}_{2}, \mathrm{P}_{2}, \mathrm{~A}_{2}$, and $\mathrm{f}_{\text {xoro }}$. $M_{2}^{\circ}\left(P_{1} \cap P_{2}\right)=M_{1}^{o}\left(P_{1}\right), M_{2}^{o}\left(p_{d}\right)=1, M_{2}^{o}\left(P_{d}^{1}\right)=0,1 \leq 1 \leq r$. Let each final marking $\mathrm{M}$ of $\mathrm{N}_{2}$ be the same as that of $\mathrm{N}_{1}$ except that $\mathrm{M}$ assigns 1 marker. to $\mathrm{p}_{\mathrm{d}}$ and zero markers to all the $\mathrm{p}_{\mathrm{d}}$, $\mathrm{s}$.

Consider any sequence $\ell^{\mathrm{n}}=\ell_{1}, \ell_{2}, \ldots \ell_{\mathrm{n}} \varepsilon \operatorname{SIMSET}_{\mathrm{N}_{1}}$. Let $\ell_{i}+1$ be the first occurrence of $t_{1}$. Clearly, the sequence $l^{i}$ can be produced by $N_{2}$, $\rightarrow$ for $1 \leq k \leq i$ $M_{2}^{k}\left(P_{1} \cap P_{2}\right)=M_{1}^{k}\left(P_{1}\right), M_{2}^{k}\left(p_{d}\right)=1, M_{2}^{k}\left(p_{d}^{j}\right)=0,1 \leq j \leq r$. When $\ell_{i+1}$ is enabled it removes a marker from each of $p_{1}, p_{2}, \ldots, p_{s} \cdot$ On completion of firing, let $\ell_{1+1}$ place one marker in $p_{t+k}$ and one in each of $p_{s+1}, \ldots, p_{t}$. Then, in, $\mathrm{N}_{2}, \mathrm{t}_{\mathrm{d}}^{\ddot{\mathrm{k}}}$ can be enabled after $\ell^{i}$. Firing of $\mathrm{t}_{\mathrm{d}}^{\mathrm{k}}$ removes one marker from each of $p_{1}, \ldots p_{s}$, one from $p_{d}$ and places a marker in $p_{d}^{k}$ enabling $t_{1}^{k}$. Firing of $t_{1}^{k}$ replaces the marker in $\mathrm{p}_{\mathrm{d}}$ and, places a marker in each of $\mathrm{p}_{\mathrm{s}+1}, \ldots, \mathrm{p}_{\mathrm{t}}$ and one marker in $p_{t+k}$. Let $M_{2}$ be the marking of $N_{2}$ after $e^{i-1} t_{d}^{k} t_{1}^{k}$. Then $M_{2}\left(P_{1} \cap P_{2}\right)$ $\mathrm{M}_{1}^{\mathrm{i}+1}\left(\mathrm{P}_{1}\right), \mathrm{M}_{2}\left(\mathrm{p}_{\mathrm{d}}\right)=1, \mathrm{M}_{2}\left(\mathrm{p}_{\mathrm{d}}^{\mathrm{j}}\right)=0,1 \leq \mathrm{j} \leq \mathrm{r}$. Continuing in this manner, if $\dot{l}_{i_{1}}, \ell_{i_{2}}, \ldots, \ell_{1_{m}}$ are occurrences of $t_{1}$ in $\ell^{n}$ then the sequence $d^{n+m}=$ $\ell^{i_{1}-1} t_{d}^{j} t_{1}^{j} l_{1_{1}+1}, \ldots, t_{d}^{j m} t_{1}^{j_{m}} \quad l_{i_{m+1}}, \ldots, \ell_{n}$ can be produced by $N_{2}$. By appropriately selecting $j_{1} \ldots, j_{m}, M_{2}^{n+m}\left(P_{1} \cap P_{2}\right)=M_{1}^{n}\left(P_{1}\right), M_{2}^{n+m}\left(P_{d}\right)-1$, $M_{2}^{n+m}\left(p_{d}^{1}\right)=0,1 \leq 1 \leq r . \quad$ By definition of $F_{2}, d^{n+m} \cdot \varepsilon \cdot \operatorname{simSET}_{N_{2}} \cdot$

Let $d^{n}=d_{1}, d_{2}, \ldots, d_{n}$ be any sequence $\varepsilon \operatorname{SIMSET}_{N_{2}}$. Let $d_{a}$ be the first occurrence of some $t_{d}^{1}$. Then the sequence $d^{a-1}$ can be produced by $N_{1} \ni M_{2}^{a-1}$ $\left(P_{1} \cap P_{2}\right)=M_{1}^{a-1}\left(P_{1}\right), M_{2}^{a-1}\left(p_{d}^{j}\right)=0,1 \leq j \leq r$. Firing of $t_{d}^{1}$ removes the marker from $p_{d}$ and one marker from each of $p_{1}, \ldots, p_{S}$. Thus $M_{2}^{a}\left(p_{j}\right)=M_{1}^{a-1}\left(p_{j}\right)-1$, $1 \leq i \leq s \cdot M_{2}^{a}\left(p_{j}\right)=M_{1}^{a-1}\left(p_{j}\right)$ for all other $p_{j}^{\prime} s$ in $P_{1} \cap P_{2}$. Clearly, no $t_{d}^{k}$ can fire until $t_{1}^{i}$ fires replacing the marker in $p_{d}$. Let $d_{b}$ be the first occurrence of $t_{1}^{i}$. The sequence $d_{1}, d_{2}, \ldots, d_{a-1}, d_{a+1}, \ldots, d_{b-1}$ can be produced by 
$N_{1} \rightarrow$ If $M$ is the marking of $N_{1}$ after this sequence then $M_{2}^{b-1}\left(p_{1}\right)=M\left(p_{1}\right)-1$, $1 \leq 1 \leq s$ and $M$ assigns the same markers to all other places in $P_{1} \cap P_{2}$. Firing of $t_{1}^{1}$ places a marker in $p_{d}$, one marker in each of $p_{s+1}, \ldots, p_{t}$ and a marker in $p_{t+1}$. Since $M_{2}^{b-1}\left(p_{1}\right) \geq 0,1 \leq 1 \leq s, M\left(p_{1}\right) \geq 1,1 \leq 1 \leq s$. Therefore $t_{1}$ can fire in $N_{1}$ at this stage. It removes a marker from each of $p_{1}, \ldots, p_{s}$. On completion of firing let the resulting marking be $M_{1}^{b}$ where $P_{t+1}$ is selected to recelve a marker. $M_{1}^{b}\left(P_{1}\right)$. $M_{2}^{b}\left(P_{1} \cap P_{2}\right), M_{2}^{b}\left(p_{d}\right)-1, M_{2}^{b}\left(p_{d}^{1}\right)=0,1 \leq 1 \leq r$. Continuing in this manner, 1 can be seen that the sequence $\ell^{\mathrm{m}}=l_{1}, \ell_{2}, \ldots, \ell_{\mathrm{m}}$ obtained by deleting the $t_{d}^{j}$ 's in $d^{n}$ and replacing the $t_{1}^{j}$ 's by $t_{1}$ can be produced by $N_{1}$. Since $d^{n} \in \operatorname{SIMSET}_{N_{2}}$, the last occurrence of $t_{d}^{j}$ in $d^{n}$ must be followed by a $t_{1}^{j}$ else $P_{d}=0$ and each final marking of $N_{2}$ assigns a marker to $\mathrm{p}_{\mathrm{d}}$. By approprlately selecting the place to receive a marker after each firing of $t_{1}$ in $N_{1}$ the marking $M_{1}^{m}$ is obtained $\ni M_{1}^{m}\left(P_{1}\right)=M_{2}^{n}\left(P_{1} \cap P_{2}\right), M_{2}^{n}\left(p_{d}\right)=1$, $M_{2}^{n}\left(\dot{p}_{d}^{j}\right)=0,1 \leq j \leq r$. By definition of $\dot{F}_{2}$, since $d^{n} \varepsilon \operatorname{SIMSET}_{N_{1}}, l^{m}$ must belong to SIMSET.

Let $I_{1}$ be any trans-labelling of $N_{1}$. Let $I_{2}$ be a trans-labelling of $N_{2}$ defined as follows: $I_{2}\left(t_{1}^{j}\right)=I_{1}\left(t_{1}\right), 1 \leq f \leq r . I_{2}(t)=I_{1}(t)$ for all other $t$ 's belonging to $T_{1} \cap T_{2}, I_{2}\left(t_{d}^{j}\right)=\emptyset, 1 \leq f \leq r$. The construction $\ell^{m}$ $\varepsilon \operatorname{SIMSET}_{\mathrm{N}_{1}}$ given $d^{\mathrm{n}} \varepsilon \operatorname{sIMSET}_{N_{2}}$ and that of $d^{\mathrm{n}+\mathrm{m}} \varepsilon \operatorname{siMSET}_{\mathrm{N}_{2}}$ given $\ell^{\mathrm{n}} \varepsilon \operatorname{SIMSET}_{\mathrm{N}_{1}}$ clearly shows that $I_{1}\left(\operatorname{SIMSET}_{\mathrm{N}_{1}}\right)=\mathrm{I}_{2}\left(\operatorname{SIMSET}_{\mathrm{N}_{2}}\right)$.

The reader can easily verify that $N_{2}$ is well behaved if $N_{1}$ is. Let $N_{1}$ have $\mathrm{k}$ transitions. If the above replacement procedure is carried out $\mathrm{k}$ times, once for each transition in $N_{1}$, we end up with a net $\mathrm{N}_{k+1}{ }^{1}, \varepsilon$, TN and interpretation $I_{k+1} \ni I_{k+1}\left(\operatorname{SIMSET}_{N_{k+1}}\right)=I_{1}\left(\operatorname{SIMSET}_{N_{1}}\right)$ and $N_{k+1}$ is well behaved if $\mathrm{N}_{1}$ is. This completes the proof of theorem 2.11.6. 
Page 25

Theorem 2.11.7: TN $=$ TN $_{\text {XORI }}$

\section{Proof:}

$\mathrm{N} \varepsilon \mathrm{TN}=>\mathrm{N} \varepsilon \mathrm{TN}_{\mathrm{XORI}}$, therefore $\mathrm{TN} \subseteq \mathrm{TN}_{\mathrm{XORI}}$ and we are only required to show that $\mathrm{TN}_{\mathrm{XORI}} \subseteq \mathrm{TN}$. Cons1der any $\mathrm{N}_{1}=\left(\mathrm{T}_{1}, \mathrm{P}_{1}, \mathrm{~A}_{1}, \mathrm{M}_{1}^{\circ}, \mathrm{F}_{1}, \mathrm{f}_{\mathrm{XORI}}^{1}\right) \cdot \varepsilon$ $\mathrm{TN}_{\mathrm{XORI}}$ : . Let $t_{1}$ be any transition of $\mathrm{N}_{1} \Rightarrow \mathrm{I}_{1}^{*}-\left\{\mathrm{p}_{1}, \ldots, \mathrm{p}_{\mathrm{S}}\right\}, \mathrm{I}_{1}^{+}=$ $\left\{p_{s+1}, \ldots, p_{s+t}\right\}, o_{1}=\left\{p_{s+t+1}, \ldots, p_{s+r}\right\}$ : Local to $t$ the net is as shown In Figure 2.11.9.

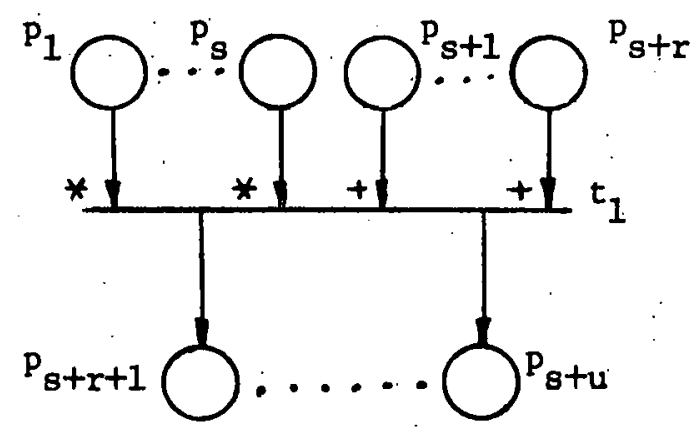

F1gure 2.11.9

Let $N_{2}=\left(T_{2}, P_{2}, A_{2}, M_{2}^{O}, F_{2}, f_{X O R I}^{2}\right)$ be the net obtained when $t_{1}$ is replaced by the net in Figure 2.11.10.

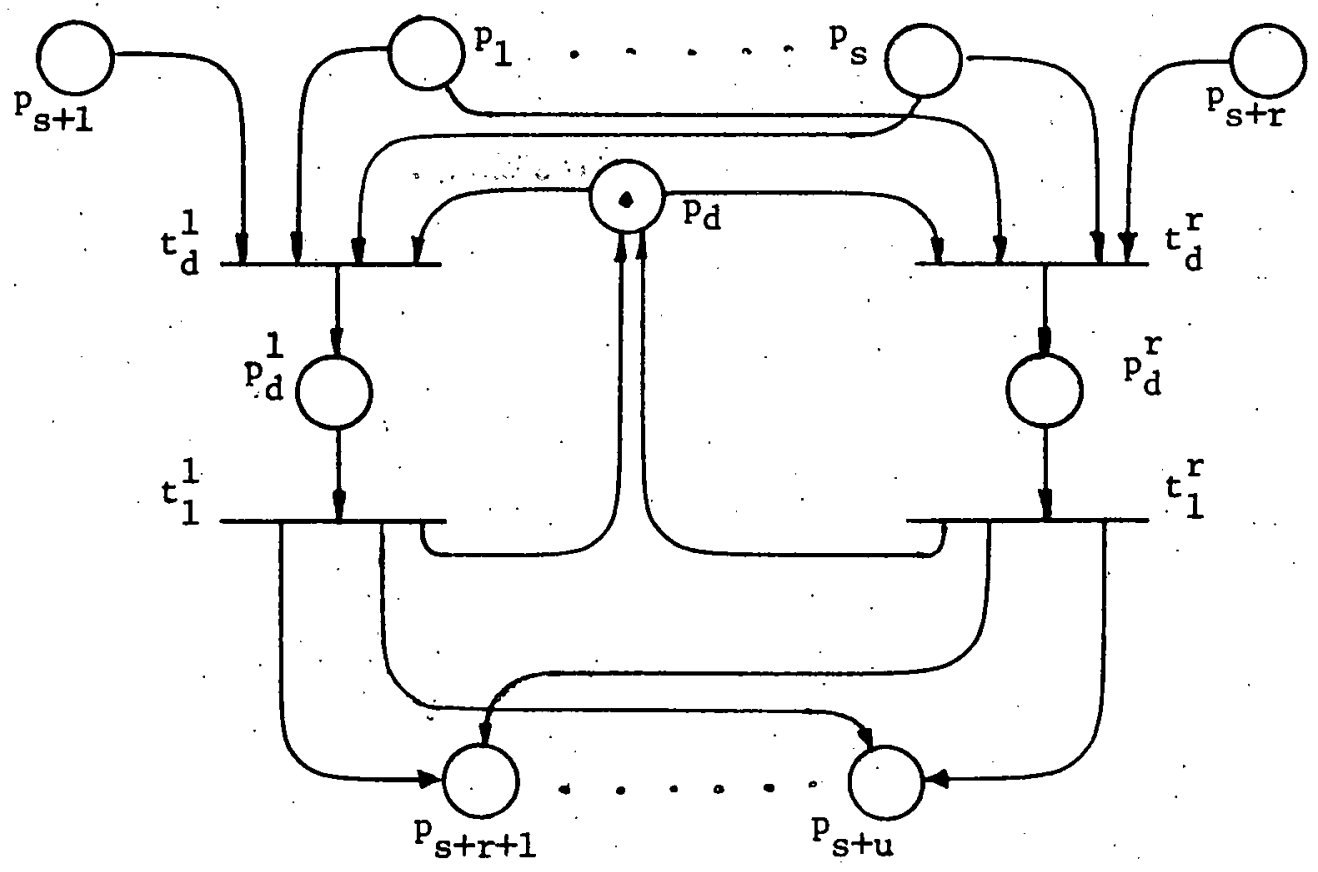


$t_{1}^{1}, \ldots, t_{1}^{r}$ correspond to $t_{1}$ in F1gure 2.11.10. $M_{2}^{o}\left(P_{1} \cap P_{2}\right)=M_{1}^{o}\left(P_{1}\right)$, $M_{2}^{0}\left(p_{d}\right)=1, M_{2}^{0}\left(p_{d}^{i}\right)=0,1 \leq 1 \leq r$. The final markings of $N_{2}$ are the same as those of $\mathrm{N}_{1}$ except that each marking in $\mathrm{M}_{\mathrm{F}}^{2}$ assigns zero markers to $\mathrm{p}_{\mathrm{d}}^{i}$, $1 \leq 1 \leq r$ and one marker to $p_{d}$. Let $I_{1}$ be any trans-labeling of $N_{1}$. Then $=\left(t_{1}^{1}\right)=I_{1}\left(t_{1}\right), 1 \leq 1 \leq r, I_{2}\left(T_{1} \cap T_{2}\right)=I_{1}\left(t_{1}\right)$ and $I_{2}\left(t_{d}^{i}\right)=\emptyset, 1 \leq 1 \leq r$.

Then an argument, similar to that in theorem 2.11 .6 can be given to prove that $I_{1}\left(\operatorname{SIMSET}_{\mathrm{N}_{1}}\right)=I_{2}\left(\operatorname{SIMSET}_{\mathrm{N}_{2}}\right)$ and $\mathrm{N}_{2}$ is well behaved if $\mathrm{N}_{1}$ is. Let $\mathrm{k}$ be the number of transitions in $N_{1}$. Then if the above replacement procedure 1s repeated $k$ times, once for each transtion in $N_{1}$, a net $N_{k+1} \varepsilon$ TN with trans-labelling $I_{k+1}$ is obtained $\ni I_{1}\left(\operatorname{SIMSET}_{N_{1}}\right)=I_{k+1}\left(\operatorname{SIMSET}_{\mathrm{N}_{k+1}}\right)$ and $\mathrm{N}_{k+1}$ is well behaved if $\mathrm{N}_{1}$ is. This completes the proof of theorem 2.11.7.

Theorem 2.11.8: $\quad \mathrm{TN}_{\mathrm{PLEX}}=\mathrm{TN}$

Proof:

$N \varepsilon \mathrm{TN} \Rightarrow \mathrm{N} \in \mathrm{TN}_{\mathrm{PLEX}}$. Therefore $\mathrm{TN} \subseteq \mathrm{TN}_{\mathrm{PLEX}}$ and we need only show that TN ${ }_{\text {PLEX }} \subseteq$ TN. We w11l lllustrate the construction and proof by means of an example. The proof for the general case is simflar. Let $N_{1}=\left(T_{1}, P_{1}, A_{1}\right.$, $\left.M_{1}^{0}, F_{1} f_{x}^{I}\right) \in T_{P L E X}$ and let $I_{1}$ be any trans-labelling. Let a particular transition $t_{1}$ have $p_{1}$ and $p_{2}$ as input places and $p_{3}$ and $p_{4}$ as output places. Let $f_{x}\left(\left(p_{1}, t_{1}\right)\right)=f_{x}\left(\left(t_{1}, p_{3}\right)\right)=1, f_{x}\left(\left(p_{2}, t_{1}\right)\right)=f_{x}\left(\left(t_{1}, p_{4}\right)\right)=2$. Thus, local to $t_{1}$, the net $N_{1}$ is as shown in Figure 2.11.11.

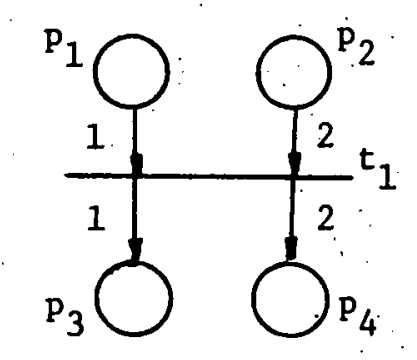

Figure 2.11.11 
The goal now is to replace $t_{1}$ by a net where the condition for any transition to fire is that no input place be empty. By defining a suitable interpretation $I_{2}$ for the net $\mathrm{N}_{2}$ obtained by the replacement, we have to ensure that $N_{2}$ produces the same process sequences as $N_{1}$ and if $N_{1}$ is well behaved then so $18 \cdot \mathrm{N}_{2}$. Each transition can then be replaced in a similar manner. and the final net will belong to the class TN. Let us try to replare $t_{1}$ by the net In Figure 2.11.12.

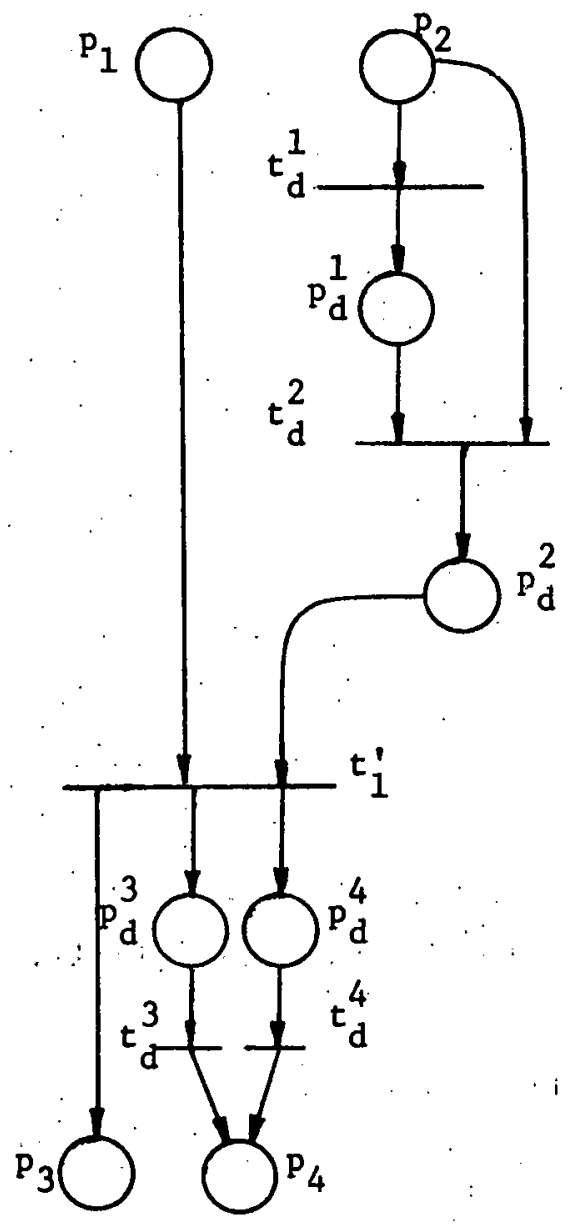

Figure 2.11.12 
Let the resulting net be $N_{2} \cdot M_{2}^{0}\left(P_{1} \cap P_{2}\right)=M_{1}^{0}\left(P_{1}\right), M_{2}^{0}\left(p_{d}^{1}\right)=0,1 \leq 1 \leq 4$. $F_{2}$ is the same as $F_{1}$ except that each marking in $F_{2}$ assigns zero markers to $\mathrm{p}_{\mathrm{d}}^{1}, 1 \leq 1 \leq 4 . \quad \mathrm{I}_{2}\left(\mathrm{~T}_{1} \cap \mathrm{T}_{2}\right)=\mathrm{I}_{1}\left(\mathrm{~T}_{1} \cap \mathrm{T}_{2}\right), \mathrm{I}_{2}\left(\mathrm{t}_{\mathrm{d}}^{1}\right)=\emptyset, 1 \leq i \leq 4, \mathrm{I}_{2}\left(\mathrm{t}_{1}^{\prime}\right)=$ $I_{1}\left(t_{1}\right)$. Thus, $t_{1}^{\prime}$ corresponds to $t_{1}$. In $N_{1}, P_{1} \geq 1$ and $P_{2} \geq 2 \Leftrightarrow t_{1}$ can fire. In $N_{2}$, the check for $P_{2} \geq 2$ is made by transitions $t_{d}^{1}$ and $t_{d}^{2}$. A marker can be placed in $\mathrm{p}_{\mathrm{d}}^{2}$ if and only if $\mathrm{P}_{2} \geq 2$. Thus $t_{1}$ can be enabled if and only if $P_{1} \geq 1$ and $P_{2} \geq 2$. When $t_{1}$ flres, it places a marker in $p_{3}$ and 2 markers in $p_{4^{-}}$. When $t_{1}^{\prime}$ fires, it places a marker in $p_{3}$ and enables $t_{d}^{3}$ and $t_{d}^{4}$, firing of which places 2 markers in $\mathrm{p}_{4}$.

Let $\ell^{n}=\ell_{1}, \ell_{2}, \ldots, \ell_{n} \varepsilon \cdot \operatorname{SIMSET}_{N_{1}}$. Let $\ell_{1}$ be the first occurrence of $t_{1}$. The sequence $e^{1-1}$ can be produced by $N_{2}$ and $M_{2}^{i-1}\left(P_{1} \cap P_{2}\right)=M_{1}^{1-1}\left(P_{1}\right)$. Since $l_{1}=t_{1}, M_{1}^{1-1}\left(p_{2}\right) \geq 2$ and $t_{d}^{1}$ can fire in $M_{2}^{1-1}$ removing a marker from $\mathrm{p}_{2}$. Since $\mathrm{M}_{2}^{1-1}\left(\mathrm{p}_{2}\right) \geq 2, \mathrm{t}_{\mathrm{d}}^{2}$ can subsequently fire placing a marker in $\mathrm{p}_{\mathrm{d}}^{2}$, and enabling $t_{1}^{\prime} . \quad t_{d}^{3}$ and $t_{d}^{4}$ are enabled by $t_{1}^{\prime}$ and on completion of firing, a marking $M$ of $N_{2}$ results $\Rightarrow M\left(P_{1} \cap P_{2}\right)=M\left(P_{1}\right)$ and $M\left(p_{d}^{1}\right)=0,1 \leq 1 \leq 4$.

Continuing in this manner, 1 t can be seen that if $\ell_{1}, \ldots l_{n} \varepsilon \operatorname{SIMSET}_{N_{1}}$ where $\ell_{a_{1}}=l_{a_{2}}=\ldots=l_{a_{m}}=t_{1}, 1 \leq a_{1}<a_{2}, \ldots, a_{m} \leq n$ then the sequence $\mathrm{d}^{\mathrm{n}+4 \mathrm{~m}}=\ell_{1}, \ldots, l_{a_{1-1}} \mathrm{~s} \ell_{a_{1+1}}, \ldots, l_{a_{m-1}} s l_{a_{m+1}}, \ldots, l_{n}$ where $s=t_{d}^{\prime}, t_{d}^{2}$, $t_{1}^{\prime}, t_{d}^{3}, t_{d}^{4}$ can be produced by $N_{2} \geqslant d_{n+4 m} \in \operatorname{SIMSET}_{N_{2}}$. By definition of $I_{2}$, $\overline{\mathrm{P}} \varepsilon \mathrm{I}_{1}\left(\operatorname{SIMSET}_{\mathrm{N}_{1}}\right) \Rightarrow \overline{\mathrm{P}} \varepsilon \mathrm{I}_{2}\left(\operatorname{SIMSET} \mathrm{N}_{2}\right)$.

Cons1der any sequence $d^{n}=d_{1}, d_{2}, \ldots, d_{n} \varepsilon \operatorname{SIMSET}_{N_{2}}$. If $t_{1}$ does not occur anywhere in $d^{n}$ and one or more of $t_{d}^{1}$ occur, then $M_{2}^{n}\left(p_{d}^{j}\right)>0$ for some $j$ and $M_{2}^{n}$ will not be covered by a final marking of $N_{2}$. If $t_{1}$ does not occur anywhere in $d^{n}$ (then neither do any $t_{d}^{i}$ 's) and the sequence $d^{n}$ can be produced by $N_{2}, M_{2}^{n}\left(P_{1} \cap P_{2}\right)=M_{1}^{n}\left(P_{1}\right), M_{2}^{n}\left(p_{d}^{1}\right)=0,1 \leq i \leq n$. Let $d_{1}$ be the first occurrence of $t_{1}$ in $d^{n}$. Then $d^{n}$ must be of the form $d^{a} t_{d}^{1} d_{a+2}, \ldots, d_{b}$ $t_{d}^{2} d_{b+2} . d_{c} t_{1}^{\prime} d_{c+2}, \ldots, d_{e}\left(t_{d}^{3}\right.$ or $\left.t_{d}^{4}\right) d_{e+2}, \ldots, d_{f}\left(t_{d}^{4}\right.$ or $\left.t_{d}^{3}\right) d_{f+2}, \ldots, d_{n}$ else once again $M_{2}^{n}$ will not be covered by any final marking. Clearly, $\ell^{c-2}=$ 
$d_{1}, \ldots, d_{a} d_{a+2}, \ldots, d_{b} d_{b+2}, \ldots, d_{c}$ can be produced by $N_{1}$ since firing $t_{d}^{1}$ or $t_{d}^{2}$ cannot enable any transition of $N_{2}$ in $T_{1} \cap T_{2} \cdot M_{1}^{c-2}\left(p_{2}\right)=M_{2}^{c}\left(p_{2}\right)+2$ and $M_{1}^{c-2}(p)=M_{2}^{c}(p)$ for all other $p \in P_{1} \cap P_{2}$. If $t_{1}^{\prime}$ can fire. In $M_{2}^{c}$ then since $M_{2}^{c}\left(p_{2}\right) \geq 0, t_{1}$ can fire in $M_{1}^{c-2}$. Firing of $t_{1}$ ylelds the following s1tuation: $M_{2}^{c+1}\left(p_{4}\right)=M_{1}^{c-1}\left(p_{4}\right)-2, M_{2}^{c+1}(p)=M_{1}^{c-1}(p)$ for all $p \varepsilon P_{1} \cap P_{2}$. Any transition in $\mathrm{T}_{1} \cap \mathrm{T}_{2}$ that can be enabled in $\mathrm{N}_{1}$ with marking $\mathrm{M}_{1}^{\mathrm{c}-1}$ can be enabled in $\mathrm{N}_{2}$ with marking $\mathrm{M}_{2}^{\mathrm{c}+1}$. Continuing this argument, it can be shown that the sequence $\ell^{f-3}=d_{1}, \ldots, d_{a} d_{a+2}, \ldots, d_{b} d_{b+2}, \ldots, d_{c}, t_{1}, d_{c+2}, \ldots, d_{e}, d_{e+2}, \ldots, d_{f}$ can be produced by $N_{1}$ and $M_{1}^{f-3}\left(P_{1} \cap P_{2}\right)=M_{2}^{f+1}\left(P_{1} \cap P_{2}\right)$ and $M_{2}^{f+1}\left(p_{d}^{f}\right)=$ $0,1 \leq 1 \leq d$. Thus, if $d^{n}$ has $m$ occurrences of $t_{1}^{\prime}$ then the sequence $l^{n-4} m$ obtained by deleting all occurences of $t_{d}^{1 / s}$ and replacing $t_{1}^{\prime}$ by $t_{1}$ can be produced by $N_{1}$ and $\left.M_{1}^{n} \quad\left(P_{1}\right)=M_{2}^{n-4} m\left(P_{1} \cap P_{2}\right), M_{2}^{n-4} m_{d}^{1}\right)=0,1 \leq 1 \leq 4$. If $d^{n} \varepsilon$ $\operatorname{SIMSET}_{\mathrm{N}_{2}}$ then by definition of $\mathrm{F}_{2}, e^{\mathrm{n}-4 \mathrm{~m}} \varepsilon \operatorname{SIMSET}_{\mathrm{N}_{1}}$. Thus, by definition of $I_{2}$, if $\overline{\mathrm{P}} \varepsilon I_{2}\left(\operatorname{SIMSET}_{\mathrm{N}_{2}}\right)$ then $\overline{\mathrm{P}} \varepsilon \mathrm{I}_{1}\left(\operatorname{SIMSET}_{\mathrm{N}_{1}}\right)$.

The above two results indicate that $I_{1}\left(\operatorname{SIMSET}_{N_{1}}\right)=I_{2}\left(\operatorname{SIMSET}_{\mathrm{N}_{2}}\right)$. The problem with this replacement procedure, however, is that $\mathrm{N}_{2}$ need not be well behaved (even though $N_{1}$ is). Let $t_{2}$ be a transition of $N_{1} \ni I_{2}=\{1,2\}$ and $f_{x}\left(\left(p_{1}, t_{2}\right)\right)=f_{x}\left(\left(p_{2}, t_{2}\right)\right)=1$. At some stage in the simulation of $\mathrm{N}_{1}$, after sequence $l^{\dot{n}}=l_{1}, \ldots, l_{n}$, let $P_{1}=1, P_{2}=2$. Assume $l_{i} \neq t_{1}, 1 \leq 1 \leq n$ and $\ddot{p}_{2}$ is not the output place of any transition. $\mathrm{d}^{\mathrm{n}}=\ell_{1}, \ldots, \ell_{\mathrm{n}}$ can be produced by $\mathrm{N}_{2}$ (as we have shown) $\Rightarrow$ after this sequence $P_{1}=1$ and $P_{2}=2$. After $d^{n}$, suppose $t_{2}$ fires in $N_{1}$, followed by the firing of $t_{d}^{1}$. Thus, the sequence $d^{n+1}=d^{n} t_{2} t_{d}^{1}$ can be produced by $N_{2}$. After this sequence, $P_{2}=0$. Since no: transition has $p_{2}$ as an output place, $p_{2}$ will remain empty. Thus, after $d^{n+1}, p_{d}^{1}$ will always contain a marker 1rrespective of simulation. Since each final marking assigns zero markers to $\mathrm{F}_{\mathrm{d}}^{1}$, there is no way of reaching a marking covered by a final marking of $\mathrm{N}_{2}$. Thus $\mathrm{N}_{2}$ is not 
well behaved.

Thus, the construction ylelds a net $N_{2}$ and interpretation $I_{2} \rightarrow I_{1}$ $\left(\operatorname{SIMSET}_{\mathrm{N}_{1}}\right)=\mathrm{I}_{2}\left(\operatorname{SIMSET}_{\mathrm{N}_{2}}\right)$. However, $\mathrm{N}_{2}$ need not be well behaved even though $\mathrm{N}_{1}$ 1s. If places $\mathrm{p}_{1}$ and $\mathrm{p}_{2}$ were not input places of any transition other than $t_{1}$, the construction would cause $N_{2}$ to be well behaved if $N_{1}$ was.

The construction can be extended so that $\mathrm{N}_{2}$ becomes well behaved. Consider the net in figure 2.11 .13 as a replacement for $t_{1}$ in $N_{1}$.

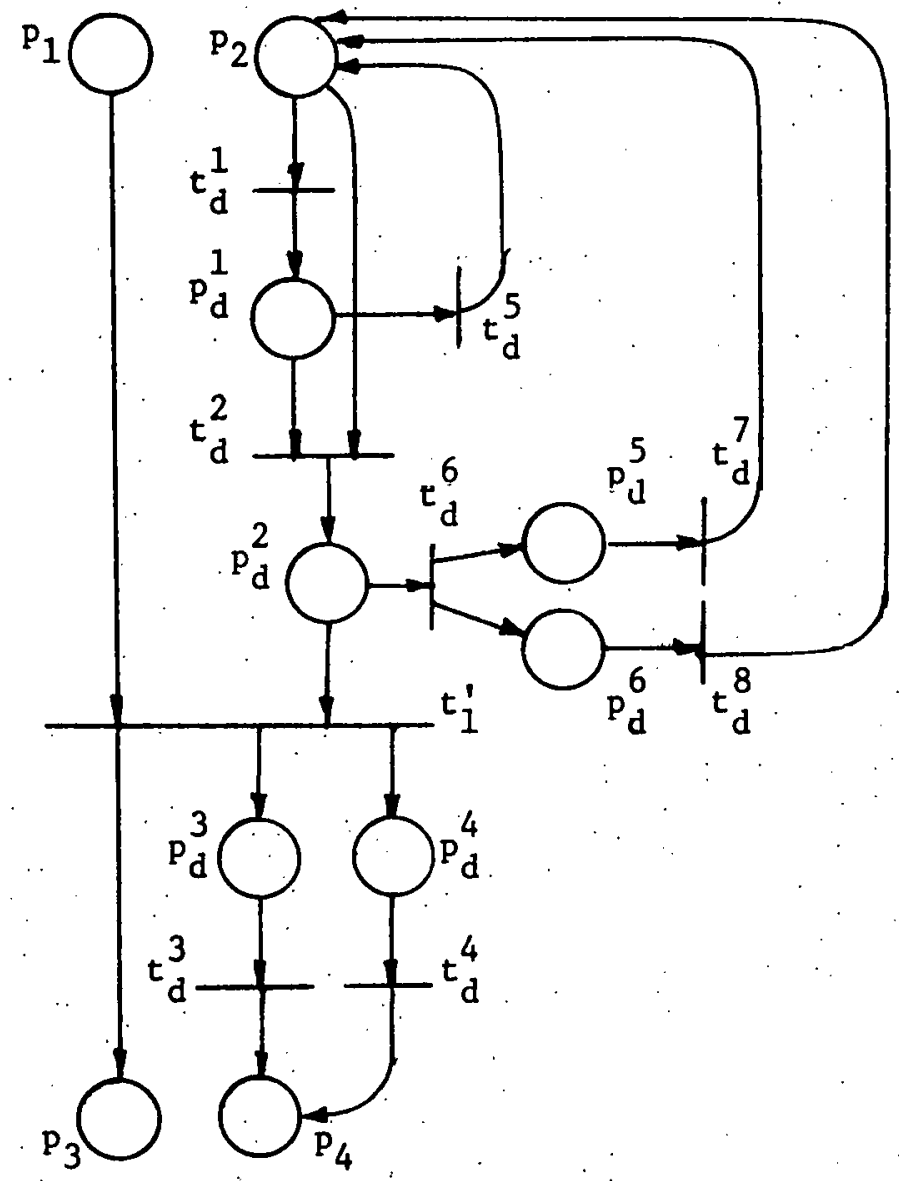

Figure 2.11.13 
Let the resuiting net be $\mathrm{N}_{2}=\left(\mathrm{T}_{2}, \mathrm{P}_{2}, \mathrm{~A}_{2}, \mathrm{M}_{2}^{\circ}, \mathrm{F}_{2}, \mathrm{f}_{\mathrm{x}}^{2}, \mathrm{M}_{2}^{\mathrm{O}}\left(\mathrm{P}_{1} \cap \mathrm{P}_{2}\right)=\mathrm{M}_{1}^{\mathrm{o}}\left(\mathrm{P}_{1}\right)\right.$, $\mathrm{M}_{2}^{\mathrm{O}}\left(\mathrm{p}_{\mathrm{d}}^{1}\right)=0,1 \leq 1 \leq 6 . \quad \mathrm{F}_{2}$ is the same as $\mathrm{F}_{1}$ except that each marking in $F_{2}$ assigns zero markers to $p_{d}^{1}, 1 \leq 1 \leq 6 . I_{2}\left(T_{1} \cap T_{2}\right)=I_{1}\left(T_{1} \cap T_{2}\right), I_{2}\left(t_{1}^{\prime}\right)$ $=I_{1}\left(t_{1}\right)$ and $I_{1}\left(t_{d}^{1}\right)=\emptyset, 1 \leq 1 \leq 8$. As before, $1 t$ can be shown that $I_{1}$ $\left(\operatorname{SIMSET} \mathrm{N}_{1}\right)=\mathrm{I}_{2}\left(\right.$ SIMSET $\left._{\mathrm{N}_{2}}\right)$. However, In this case, if $\mathrm{N}_{1}$ is well behaved, then so is $\mathrm{N}_{2}$. In the previous construction, $\mathrm{N}_{2}$ was not well behaved because checking if $P_{2}$ was greater than 1 was achieved by the sequential firing of two transitions $t_{d}^{1}$ and $t_{d}^{2}$ : Thus, if $p_{1}$ and $p_{2}$ were also input places of other transitions then "dead-1ock" could occur if the same replacement technique is used for the other transitions. The replacement in figure 2.11.13, however, avolds this. At each step of the checking procedure, it is possible to revert back to the start of the check. Thus, If $P_{2}>1$; then the check can be completed and $t_{1}^{\prime}$ can be enabled. If $P_{2}=1$, firing of $t_{d}^{1}$ will not lead to dead lock becuase $t_{d}^{5}$ can fire causing the marker in $p_{2}$ to be replaced. At each stage in the simulation of $N_{2}$, elther a transition in $\left(T_{1} \cap T_{2}\right)$ is fired or $t_{d}^{1}$ followed by $t_{d}^{2}$ can be fired enabling: $t_{1}^{\prime}$ or a sequence of $t_{d}^{1}$ 's can be fired returning the net to the marking at the start of the stage. It can easily be seen now that if $\mathrm{N}_{1}$ is well behayed. $\mathrm{N}_{2}$ must also be well behaved.

For the general case, a transition $t_{1}$ having input places $p_{1}, \ldots, p_{s}$ and output places $p_{s+1}, \ldots, p_{s+t} \Rightarrow f_{x}\left(\left(p_{1}, t_{1}\right)\right)=n_{1}, 1 \leq 1 \leq s$ and $f_{x}\left(\left(t_{1}, p_{1}\right)\right)$ $=\mathrm{n}_{1}, \mathrm{~s}+1 \leq 1 \leq \mathrm{t}$, can be replaced by the net in figure 2.11 .14 to y1eld a net $\mathrm{N}_{2}$ : 
Page 32

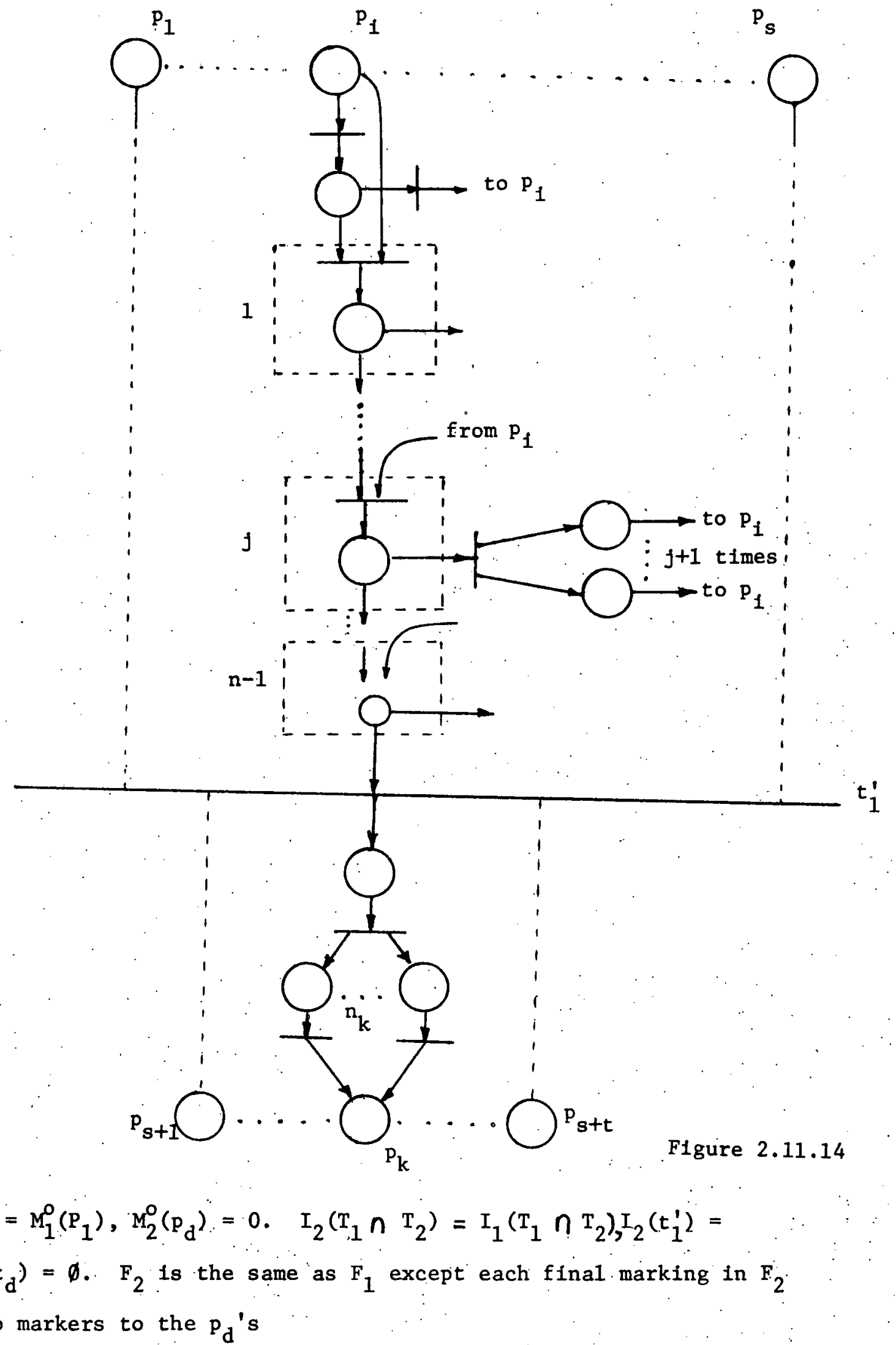


As we have shown, $I_{2}\left(\operatorname{SIMSET}_{2}\right)-I_{1}\left(\operatorname{SIMSET}_{N_{1}}\right)$ and $N_{2}$ is well behaved if $N_{1}$ is.

If $\mathrm{N}_{1}$ contains $\mathrm{k}$ transitions, the replacement procedure can be carried out

$k$ times once for each transiton of $\mathrm{N}_{1}$ to yield a net $\mathrm{N}_{k+1}$ and interpretation $I_{k+1} \ni I_{1}\left(\operatorname{SIMSET}_{N_{1}}\right)=I_{k+1}\left(\operatorname{SIMSET}_{N_{k+1}}\right)$ and $N_{k+1}$ is well behaved if $N_{1}$ is.

3. Summary

We have defined nine classes of Petri nets and have established the following hiçrarchy :

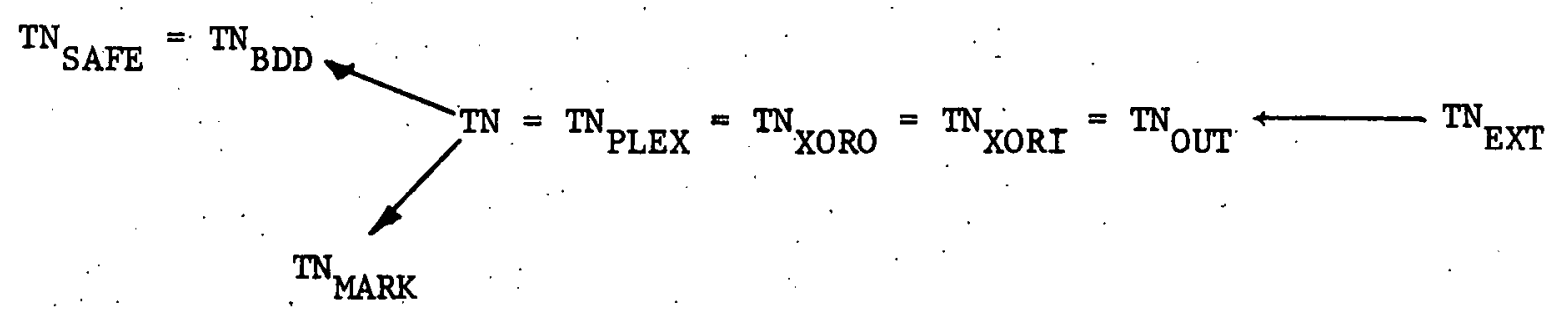

As w\$1l be demonstrated in subsequent chapters, this hierarchy will be useful In establishing the capab1litles of different controlling agents. For example, we will show that the complex Bilogic Directed Graph model of UCLA, Petersons P-nets and ordinary Petri nets are equivalent with respect to their power for representing coordinations. We feel this is a significant result and is derived directly from the above hierarchy. 


\section{Controlling agents in different models}

\subsection{U.C.L.A. Btudies}

In the past decade, a vast amount of literature (on graph models of computations) has emerged from U.C.L.A. as an outgrowth of the variable structure computer project. The intent of the first model by Estrin and Turn was to give a representation of programs to be run on a variable structure computer. Over the years, the model has been extended and refined and different aspects have been studied by different investigators. For a good survey, the reader is referred to [6]. We will proceed directly to the latest and most general model, the Graph Model of Computation from U.C.L.A., studied by Cerf in his doctoral dissertation, [10]. This Graph Model of Computation 1s based on the works of Martin [17], Baer [5], Bovet [9], Russel [22], Volansky [24], Gostelow [12], Slutz [23], and Holt [13]. The main objectlve of Cerf's dissertation was to seek methode for analyzing program control flow based un its representation in the model, to study the practical implications of the model, and to discover potentially correctable control anomalies such as deadlock, etc.

The basic controlling agent in the Graph Model of Computation is the Complex, Bllogic Directed Graph. We will proceed with the definitions as presented in [10]:

Definition 4.1.1: A complex directed graph (digraph) is a pair (V,A) where

(1) $V$ is a finite set of vertices of cardinality | V |

(2) A is a finite set of arcs and if a $\varepsilon$ A then a is an ordered pair of sets 


$$
\begin{aligned}
& \mathrm{a}=(\mathrm{I}, \mathrm{J}) \\
& \mathrm{I} \subseteq \mathrm{V}, \mathrm{J} \subseteq \mathrm{V}
\end{aligned}
$$

(3) If $I=\{1\}, J=\{\}\}$ we write $a=(1, j)$

(4) We permft I $=\phi$ or $J=\phi$, but not both

(5) When $|\mathrm{J}|>1$ we have a mult1-headed arc

(6) When $|I|>1$ we have a multi-talled arc.

Definition 4.1.2: A complex, multiple-entry, multiple-exit digraph is a four-tuple, $G=(V, I, T, A)$ where

1. $(V, A)$ is a digraph

2. I is a set of entry arcs

3. T is a set of exit arcs.

Definition 4.1.3: A complex, single-entry, single exit digraph (SESX) is a MEMX, $G=(V, I, T, A)$ such that

$$
\begin{aligned}
& \text { 1. } I=\{s\} \text { and } s=\left(\phi, v_{1}\right) \\
& \text { 2. } T=\{x\} \text { and } x=\left(v_{f}, \phi\right)
\end{aligned}
$$

Definition 4.1.4: A complex, bllogic, directed graph (bigraph) is a triple. (G, L, Q) where:

1. $G=(V, A)$ is a SESX digraph

2. $L=\left(L^{-}, L^{+}\right)$is a total, single-valued mapping from the set of vertices, $v$, into the set $\{+, *\}$.

3. $Q=\left(Q^{-}, Q^{+}\right)$is a partial single valued mapping from $V \times A$ into the integers.

The simulation rules are given by Cerf in terms of a Token Machine after defining the graph model of computation (GMC) which 18 semilnterpreted and then defining an interpretation. Without going into these detalls, we will present the simulation rules informally here: 
Definttion 4.1.5: The inbranching set of vertex $v$ is $A^{-}(v)=\{a \mid$ a $E A$ and $a=(I, J)$ and $v \in J\}$.

Definition 4.1.6: The outbranching set of vertex $v$ is $A^{+}(v)=\{a \mid$ a $\varepsilon A$ and $a=(I, J)$ and $v \in I\}$.

A simulation starts by placing one "token" on the entry arc. All other arcs have zero tokens. At any stage in a simulation let $N(a)$ be the number of tokens on arc a. A vertex $v$, with $\mathrm{L}^{-}(\mathrm{v})=+$ can Initiate if $\exists$ a $\varepsilon A^{-}(v), N(a) \geq Q^{-}(v, a)$. When $1 t$ inftiates, it removes $Q^{-}(v, a)$ tokens from a. A vertex $v$ with $L^{-}(v)=$ * can inftiate if for all a $\varepsilon A^{-}(v)$, $N(a) \geq Q^{-}(v ; a)$. When it initiates, it removes $Q^{-}(v, a)$ tokens from each a $E A$. When $v$ terminates, if $\mathrm{L}^{+}(\mathrm{v})=+$ then arbitrarily, a particular a $\varepsilon A^{+}(v)$ is selected and $Q^{+}(v, a)$ tokens are placed on a. When $v$ terminates, if $L^{+}(v)=*$ then $Q^{+}(v, a)$ tokens are placed on each arc a $\varepsilon A^{+}(v)$. Initiations and terminations cannot occur simultaneously. The graph terminates when no vertex can Inftlate or terminate.

A computation sequence is a sequence of Inftiations and terminations at the end of which no vertex can Inftlate or terminate. An inftiation of $v$ corresponds to the symbol $\bar{v}$ and a termfnation of $v$ to the symbol $v$. Cerf also defines a properly terminating graph where one of the conditions is that whenever the graph terminates, it terminates such that there is a token on the exit arc and no tokens on any other arc. Thus, it seems reasonable to define a simulation sequence of such a graph as a sequence of initiations and terminations that, starting with a single token on the entry arc, causes the graph to end up with one token on the exit arc and no tokens elsewhere. The set of all sequences of a bigraph B is 
called $\mathrm{S}_{\mathrm{B}} \cdot$ According to our definitions then, a graph 1s well behaved If It satisfles the condition for proper termination stated above.

Figure 4.1.1 1s an example of a bigraph.

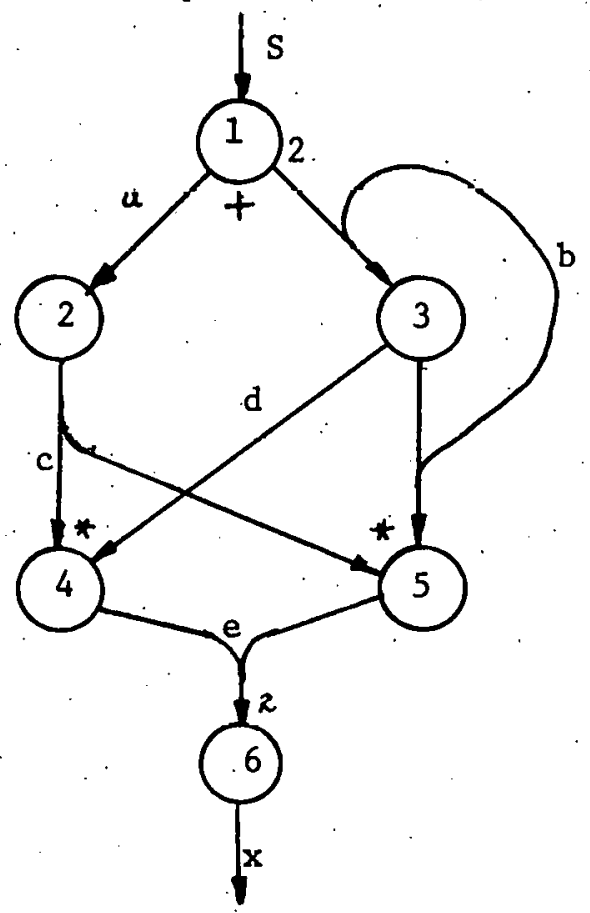

$Q^{+}(1, b)=2=Q^{+}(2, c)=Q^{-}(6, e)$

$Q^{-}(1, s)=1=Q^{+}(1, a)=Q^{-}(2, a)=Q^{-}(3, b)=Q^{+}(3, b)=Q^{+}(3, d)$

$=Q^{-}(4, c)=Q^{-}(4, d)=Q^{+}(4, e)=Q^{+}(5, e)=Q^{+}(6, x)$. (The values, other than 1 , are Indicated by small numerals).

F1gure 4.1.1 
Given a bigraph B, it is easy to construct trans-labelled ordinary Petri net $\mathrm{N}$ which produces the same sequences as $B$. Let us proceed in stages. Stage A. Each arc of $B$ corresponds to a place of $N$, each node of $B$ corresponds to a transition of $\mathrm{N}$. An arc is drawn from a place $\mathrm{p}$ to a transition $t$ if and only if the arc corresponding to $p$ is in the inbranching set of the node corresponding to transition $t$. An arc is drawn from a transition to to a place $p$ if and only if the arc corresponding to $p$ is in the outbranching set of the node corresponding to $t$. $M^{0}$, the Intial marking of $\mathrm{N}$ is defined as follows:

$$
M^{0}(x)= \begin{cases}1 & \text { if } x \text { corresponds to the entry arc } \\ 0 & \text { otherwise. }\end{cases}
$$

The set of final markings $F=\left\{F_{1}\right\}$ where

$$
F_{1}(x)= \begin{cases}1 & \text { if } x \text { corresponds to the exit arc } \\ 0 & \text { otherwise. }\end{cases}
$$

Thus, corresponding to the graph. B in Figure 4.1.1, we get a net $\mathrm{N}$ shown in Figure 4.1.2.

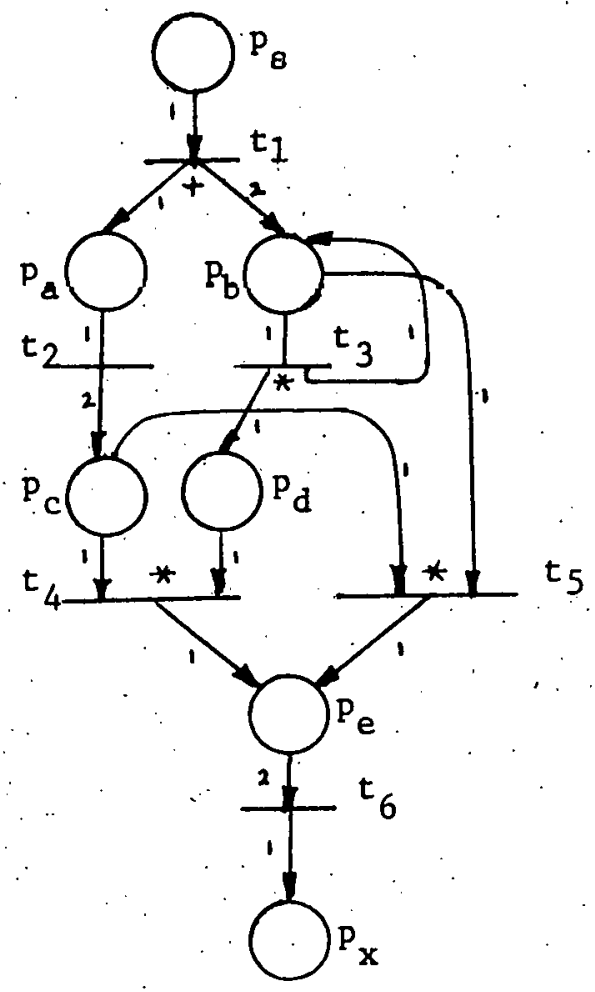

Figure 4.1 .2 


$$
\begin{aligned}
& M^{0}\left(p_{8}\right)=1 \\
& M^{0}(p)=0, \quad p \neq p_{8} \\
& F=\left\{F_{1}\right\}
\end{aligned}
$$$$
F_{1}\left(p_{x}\right)=1
$$$$
F_{1}(p)=0, p+p_{x}
$$

The net obtalned does not really fall into any of the 9 classes discussed earifer but 18 really a combination of TN $_{\text {PLEX }}$. TN XORO $_{\text {, TN }}$ XORI The input and output logics are indicated. A numeral next to arc $(t, p)$ Indicates the number of markers placed in $p$ when terminates. A numeral near ( $p, t)$ indicates the number of markers that must be in $p$ before $t$ can Initiate. This 18 also the number of markers removed from $p$ when $t$ is enabled.

Stage B. In order to be able to talk of inftiations and terminations separately, each transition in the net (obtained in stage A) is split Into two as Indicated in figure 4.1.3.
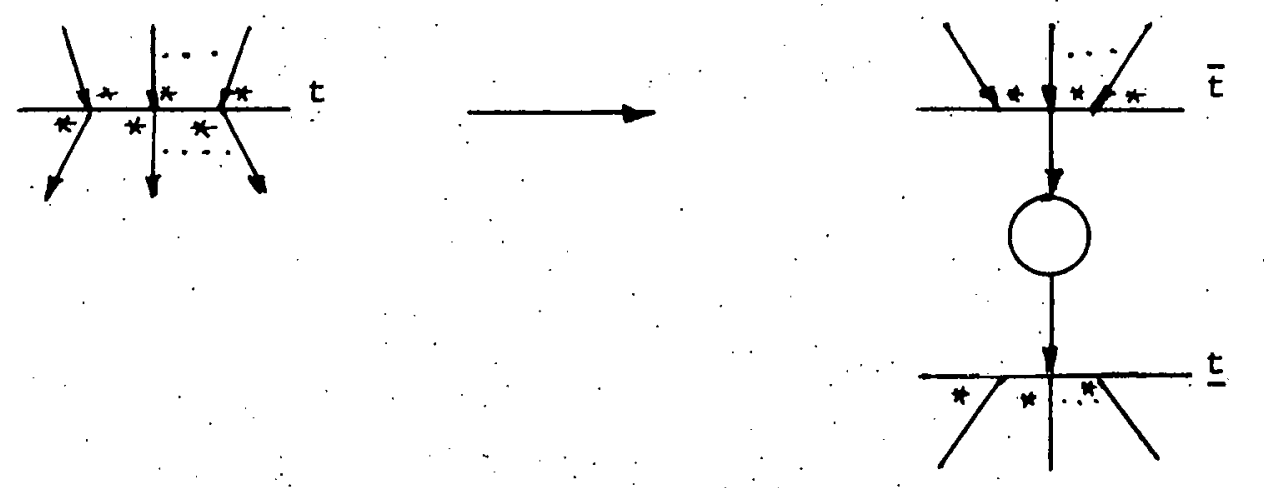

Figure 4.1 .3 (contd. on next page) 
F1gure 4.1 .3 (contd.)
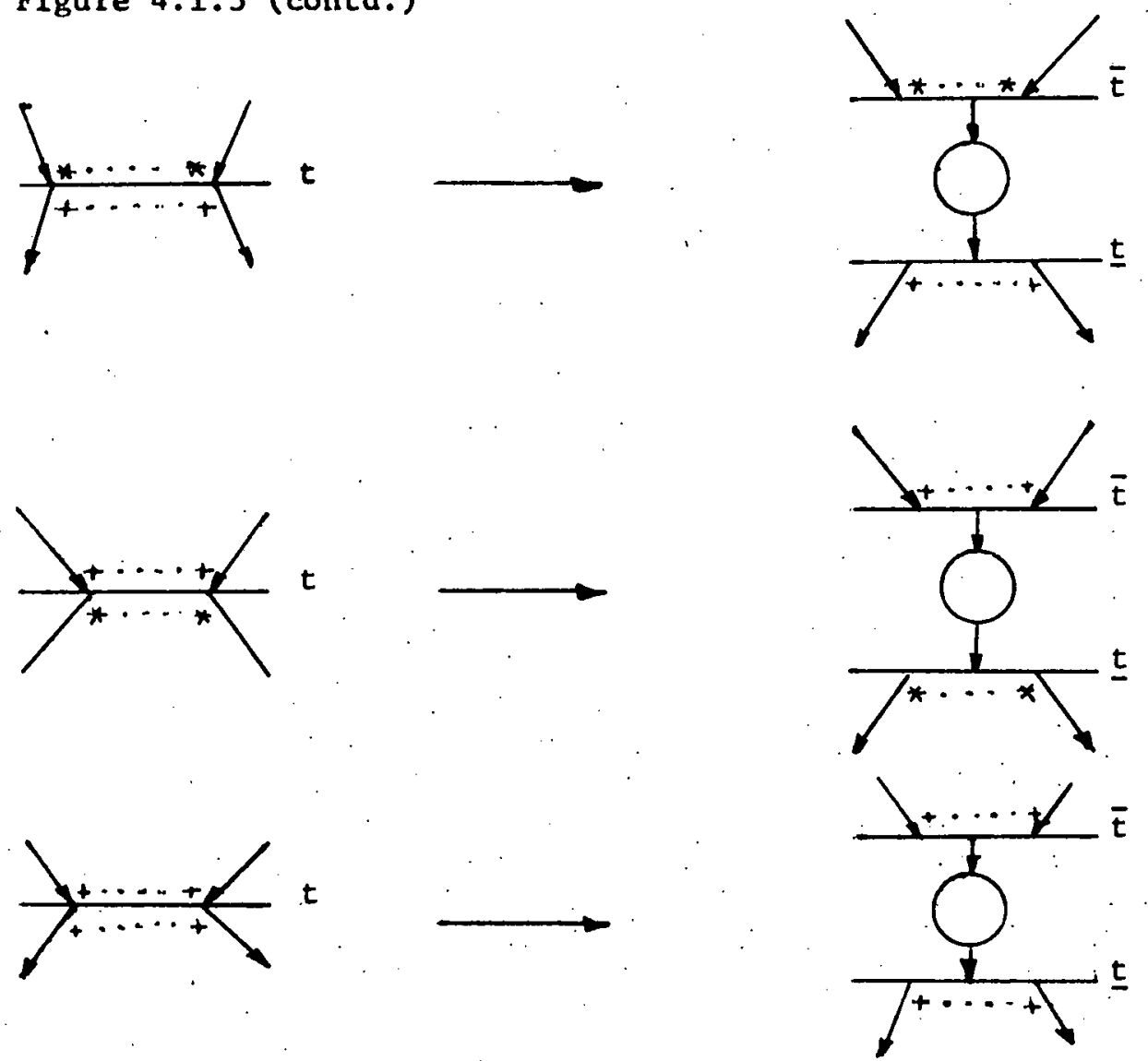

Let the new net obtalned be $N_{1}=\left(T_{1}, P_{1}, A_{1}, M_{1}^{0}, F^{1}, f_{e}^{l}\right)$. Define a trans-1abelling $I_{1} \rightarrow I_{1}(\bar{t})=\vec{v}$ and $I_{1}(t)=\underline{v}$ where $t$ corresponds to vertex $v$ of $B$. Let $X$ be the set of new places introduced in $N_{1}$. Then $M_{1}^{0}$ is the same as $M^{0}$ except that all places in $X$ are assigned zero markers. $F^{1}$ is the same as $F$ except that the final marking $M$ in $F^{l}$ assigns zero markers to each place in $X$. It should be obvious that $I_{1}\left(\operatorname{SIMSET}{ }_{N_{1}}\right)=S_{B}$, and $N_{1}$ is well behaved if $B$. is. For the net $N$ in Figure 4.1.2, we get the net $N_{1}$ shown in Figure 4.1.4. The numerals near the arcs have the same meanings as before. 
Page 41

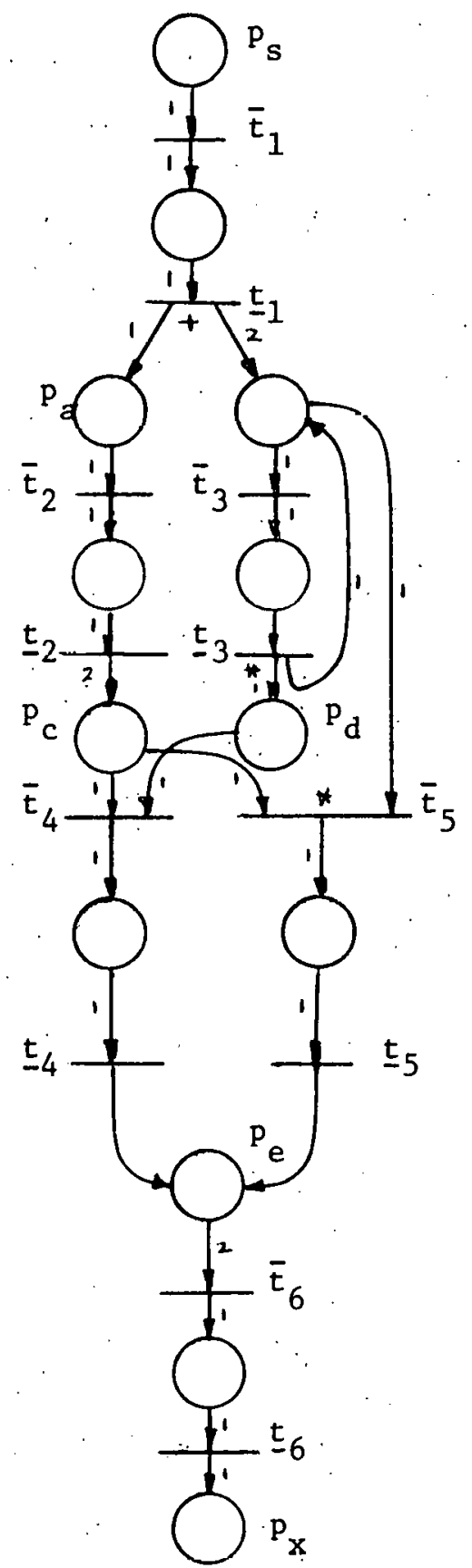

Figure 4.1.4 
Theorems 2.11 .6 and 2.11 .7 and the respective stage by stage replacements indicate that a net $\mathrm{N}_{2}=\left(\mathrm{T}_{2}, \mathrm{P}_{2}, \mathrm{~A}_{2}, \mathrm{M}_{2}^{0}, \mathrm{~F}^{2}, \mathrm{f}_{\mathrm{e}}^{2}\right) \varepsilon \mathrm{TN}_{\mathrm{PLEX}}$ can be constructed with trans-labelling $I_{2} \rightarrow I_{2}\left(\operatorname{SIMSET}_{N_{2}}\right)=I_{1}\left(\operatorname{SIMSET}_{\mathrm{N}_{1}}\right)$ and $\mathrm{N}_{2}$ is well behaved if $\mathrm{N}_{1}$ is. In particular, the replacements to be used to obtain $\mathrm{N}_{2}$ are shown in Figure 4.1.5.
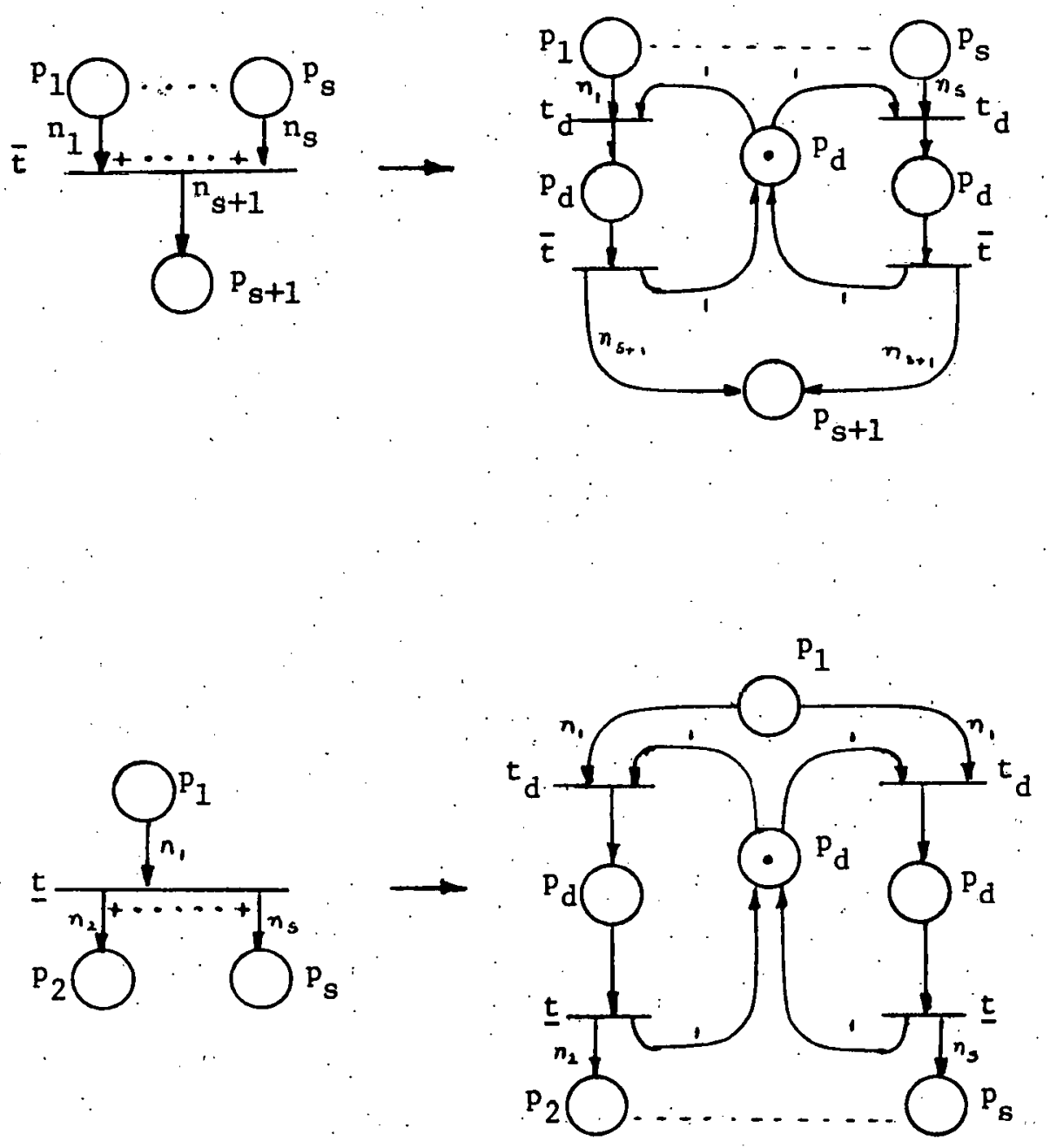

F1gure 4.1.5

$\mathrm{M}_{2}^{0}, \mathrm{~F}^{2}$ and $\mathrm{I}_{2}$ can be suitably defined so that $\mathrm{I}_{2}\left(\operatorname{SIMSET}_{\mathrm{N}_{2}}\right)=$ $I_{1}\left(\operatorname{SIMSET} N_{1}\right)$ and $N_{2}$ is well behaved if $N_{1}$ is. We can now directly apply Theorem 2.11.8 to conclude that there exists a net $\mathrm{N}_{3} \varepsilon \mathrm{TN}_{\mathrm{PLEX}}$ and 
trans-1abelling $I_{3} \rightarrow I_{3}\left(\operatorname{SIMSET}_{\mathrm{N}_{3}}\right)=\mathrm{I}_{2}\left(\right.$ SIMSET $\left._{\mathrm{N}_{2}}\right)$ and $\mathrm{N}_{3}$ is well behaved 1f $\mathrm{N}_{2}$ 18. Thus, there exists a net $\mathrm{N}_{3} \in \mathrm{TN}_{\text {PLEX }}$ and trans-labeliling $I_{3} \rightarrow I_{3}\left(\operatorname{SIMSET}_{\mathrm{N}_{3}}\right)=\mathrm{S}_{\mathrm{B}}$ and $\mathrm{N}_{3}$ is well behaved if $\mathrm{B}$ is.

To study the capabilities of $\mathrm{CBD}$ graphs, we need not be concerned with Initiations and terminations of computations but with process sequences. We can also generallze the CBD by considering a triple $((V, A), L, Q)$ where $(V, A)$ is a digraph. Initial and final markings can be appropriately defined, where an inftial marking is an inftial distribution of tokens on arcs and final markings are final distributions of tokens on arcs where for each final marking the number of tokens on some arcs may be left unspectified. The terms "trans-labelling", "simulation sequence", "process sequence" and $I\left(\right.$ SIMSET $\left._{B}\right)$ can be defined as in Section 2.11 .

Clearly, given any such graph $B$ and trans-labelling $I^{\prime}$ a net $N \varepsilon$ TN can be constructed with trans-labelling $I \Rightarrow I\left(\operatorname{SIMSET}_{N}\right)=I$ ! ( $\left(\operatorname{SIMSET}_{B}\right)$. and $N$ is well behaved if $B$ is. The construction is similar to that described earlier in this section. Also, given any $N \in T N$ and trans-iabelling $I$, a graph $B$ can be constructed with trans-labelling $I^{\prime} \rightarrow I^{\prime}\left(\right.$ SIMSET $\left._{B}\right)=$ $I\left(\operatorname{SIMSET}_{\mathrm{N}}\right)$ and $\mathrm{B}$ is well behaved if $\mathrm{N}$ is. Here also, places of $\mathrm{N}$ correspond to mult1-head/multi-tail arcs of $B$ and transitions of $N$ to vertices of B.

The above discussion establishes that the controlling agent in bigraphs is essentlally an ordinary Petri net and thus has the same capab1lities and limitations.

\subsection{The Karp and Miller Program Graph Model}

This model was proposed by Karp and Miller [14] in 1966 to describe and analyze parallel computations. The emphasis in the report was to 
show that each graph represented a unique computation and to investigate termination properties. Data queues are associated with arcs in the graph. Nodes represent computation steps. Whether a particular step can be inftiated or not depends on the lengths of the queues on branches directed Into the associated node. The type of control is severely restricted in that each queue has a unique "source" and a unique "sink". node. Data dependent conditional transfers cannot be represented. More formally, a computation graph $G$ is defined as follows:

Definition 4.2.1: A computation graph G is a directed graph consisting of :

(1) nodes $n_{1}, n_{2}, \ldots \ldots \ldots, n_{\ell}$;

(11) branches $d_{1}, \ldots \ldots, d_{t}$, where any branch $d_{p}$ is directed from a specifled node $n_{i}$ to a specified node $n_{j}$;

(1ii) four nonnegative integers $A_{p}, U_{p}, W_{p}, T_{p}$, where $T_{p} \geq W_{p}$, associated with each branch $d_{p}$.

For $d_{p}=\left(n_{1}, n_{j}\right)$, the parameters have the following interpretation:

$A_{p}$ gives the number of data words initially in the first-in, firstout queue associated with $d_{p} \cdot U_{p}$ gives the number of words added to the queue whenever the operation $o_{i}$ associated with $n_{1}$ terminates; $W_{p}$ gives the number of words removed from the queue whenever the operation $o_{j}$ is Inftiated; and $T_{p}$ is a threshold giving the minimum queue length of $d_{p}$ which permits the initiation of $0_{f}$.

In the uninterpreted version of this model the arcs can be considered to carry markers rather than be associated with queues of data. Removal and placing of data then correspond to removal and placing of markers. The nodes can have processes, associated, rather than steps of an operation. 
Page 45

If each node is replaced by a "transition". and in each arc, a "place" is inserted to hold the markers, then the resulting net closely resembles the marked graph model.

For any graph G, final markings, simulation sequences, trans-1abelling, process sequences, I(SIMSET ${ }_{G}$ ) etc. can be defined as in Section 2.11.

Let the following restrictions be placed on the parameters $A_{p}, U_{p}$, $T_{p}$ and $W_{p}: A_{p}=0$ or $1, U_{p}=W_{p}=T_{p}=1$ for all arcs $d_{p}$. Let the resulting class of graphs be called GR1. Then clearly the control representable by a graph $G \in G R 1$ can be represented by a marked graph and vice-versa. Thus, GRI $=\operatorname{TN}_{\text {MARK }}$.

Let $A_{p}, U_{p}, W_{p}, T_{p}$ be nonnegative integers, but let $W_{p}$ be restricted to be equal to $T_{p}$. Let the resulting class of graphs be GR2. Th1s class of graphs has already been studied in the proof of Theorem 2.11.8. Notice that a graph $G \varepsilon$ GR2 can be considered to be a net $N \varepsilon$ TN PLEX with the restriction that each place has exactly one input and one output transition. It was shown in Theorem 2.11 .8 that the control provided by such a net $N$ can also be provided by a net $N^{\prime} \varepsilon$ TN. However, as in the proof of Theorem 2.11 .4 , it can be shown that GR2 cannot represent all the coordinations representable by TN. Thus GR2 lies between $\mathrm{TN}_{\mathrm{HARK}}$ and TN in our hierarchy.

Let us now consider the unrestricted model. Figure 4.2.1 gives a node in some particular graph G.

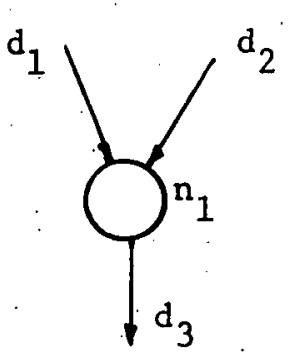

$$
\begin{aligned}
& \mathrm{A}_{1}=\mathrm{A}_{2}=\mathrm{A}_{3}=0 \\
& \mathrm{U}_{1}=\mathrm{U}_{2}=1 \\
& \mathrm{~T}_{1}=3, \mathrm{~T}_{2}=2 \\
& \mathrm{~W}_{1}=2, \mathrm{~W}_{2}=1 \\
& \mathrm{U}_{3}=2, \mathrm{~W}_{3}=\mathrm{T}_{3}=1
\end{aligned}
$$


Page. 46

Replacing arcs by places and the node by a transition ylelds the net in Figure 4.2 .2 .

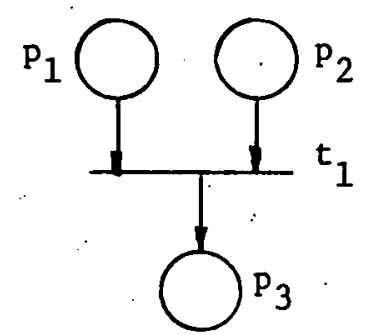

Figure 4.2 .2

If $W_{1}=3$ and $W_{2}=2$ then the replacement shown in Figure 4.2.3 (1gnoring dotted 1ines) is a valid one as demonstrated in the proof of Theorem 2:11.8.

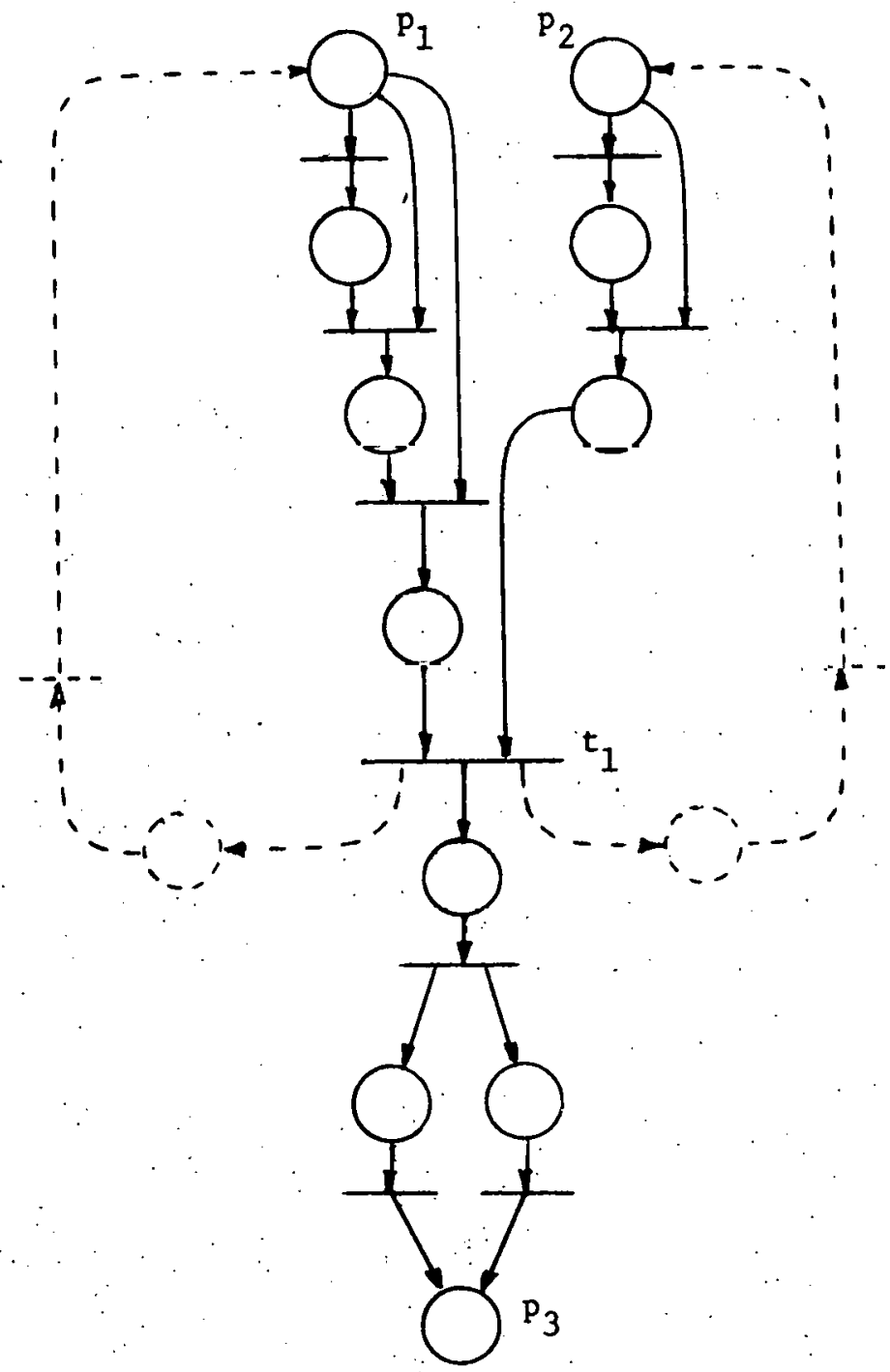

Figure 4.2.3 
If $W_{1}=2$ and $W_{2}=1$ then after the test for $P_{1} \geq 3$ and $P_{2} \geq 2$ is made, 1 marker must be replaced in each of $p_{1}$ and $p_{2}$. This is achieved by the dotted lines in Figure 4.2.3. Since the difference $T_{p}-W_{p}$ is always finite and a priori known, after the tests for initiation are made and a particular transition enabled, $T_{p}-W_{p}$ markers can be replaced in each place representing arc $d_{p}$. Thus, the control representable by any graph in the Karp and Miller program graph model can be represented by an ordinary Petri net. The reverse, however, is not true as can be shown in a manner similar to the proof of $\mathrm{TN} \rightarrow \mathrm{TN}_{\mathrm{MARK}}$. Thus the control of the Karp and Miller program graph model is less powerful than ordinary Petri nets and lies between TN ${ }_{\text {MARK }}$ and TN in our hierarchy.

\subsection{The Karp and M11ler paralle1 program schemata}

The parallel program schema introduced and studied by Karp and Miller in 1968 [15] consists essentially of: a set $M$ of memory locations; a finfte set $A$ of operations; and $T$, a control for sequencing the initiations and terminations of operations. Associated with each operation a $\varepsilon A$ is a set of domain locations $D(a) \subseteq M$ and a set of range locations $R(a) \subseteq M$. In 1ts unrestricted form, $T$ is a very general control defined as a four-tuple. $\left(Q, q_{0}, \Sigma, \tau\right)$ where $Q$ is a set of states, $q_{0}$ is the Initial state, $\sum$ is an alphabet containing, for each a $\varepsilon A$, an initiation symbol $\bar{a}$ and the termination symbols $a_{1}, a_{2}, \ldots \ldots a_{K(a)} . \tau$ is a partial transiton function from $Q \times \sum$ to $Q$ which is total on termination symbols. If the control is in some state $q_{i}$ then some operation a $\varepsilon$ A can initiate If $t\left(q_{i}, \bar{a}\right)$ is defined. On inftiation the control proceeds to state $\tau\left(q_{1}, \bar{a}\right)$. Once an operation a initiates, 1 t obtains its operands from $D(a)$ and at some later time places the results in $R(a)$. The model can 
represent conditional transfers since each operation a can terminate with any one of the symbols $a_{1}, \ldots \ldots \ldots \ldots, a_{K(a)}$. Also, several inftiations of the same operation are possible before termination. More formally, Definition 4.3.1: A (parallel program) schema $S=(M, A, T)$ is specified by

(1) A set $M$ of memory locations

(2) A fintte set $A=\{a, b, \ldots \ldots\}$ of operations and for each operation a in A:

(1) a positive integer $K(a)$ called the number of outcomes of $a$;

(11) a set $D(a) \subseteq M$ whose elements are the domaln locations for a;

(111) a set $R(a) \subseteq M$ whose elements are the range locations for $a$.

(3) A quadruple $T=\left(Q, q_{0}, \Sigma, \tau\right)$ called the control where:

(1) $Q$ is a set of states;

(11) $q_{0}$ is a designated state called the initial state;

(111) $\Sigma$, the alphabet, is the union of $\Sigma_{1}=\bigcup_{a \in A}\{\bar{a}\}$ the Initiation symbols, and $\Sigma_{t}=\bigcup_{a \in A}\left\{a_{1}, a_{2}, \ldots \ldots, a_{K(a)}\right\}$ the termination symbols.

(iv) $\tau$, the transition function, is a partial function from $Q \times \Sigma$ Into. $Q$ which is total on. $\Sigma_{t}$.

clearly, $T$ is an extremely general control since absolutely no restrictions are placed elther on the cardinalfty of $Q$ or on the transition function $\tau$. The specification of $T$ in general does not consist of a "finite description". We will thus move directly to more restricted controls studied by Karp and Miller.

Definition 4.3.2: $: \ddot{S}=(M, A, T)$ is a finite state schema if $S$ is a schema that has a finite number of states. 
We have already established the equivalence of finite state automata and safe Petri nets earlier in this report, in the sense that every regular language can be generated by a trans-labelled safe Petri net and vice versa. Thus, the fintte state schema of Karp and Miller $=\mathrm{TN}_{\mathrm{SAFE}}{ }^{\circ}$. However, we will illustrate with an example [18] the construction of a Petri net, given a finite state control $T$ of the Karp and Miller schema. This example also illustrates how sequences of initiations and terminatIons (rather than process sequences) are modeled using Petri nets. Example 4.3.1

The graph in Figure 4.31. Is the control $T$ of a Karp and Myller parallel program schema.

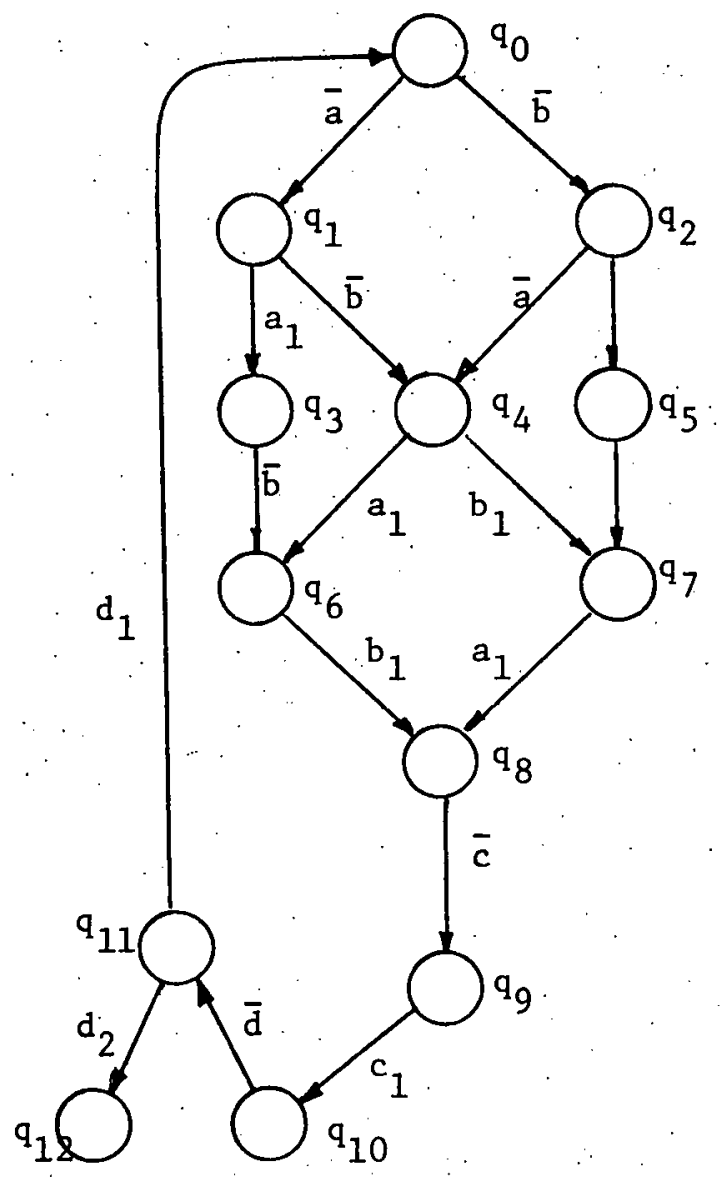

Figure 4.3.1 
Page 50

Each of the operations $a, b, c$ has only one possible outcome, $d$ has two. Note that the control specifies that $a$ and $b$ can proceed concurrently and c can proceed only on completion of $a$ and b. The schema thus represents a simple fork-join pair.

A trans-labelled Petri net representing the same coordination and that has an obvious one to one correspondence with the net in Figure 4.3 .11 . given in Figure 4.3.2. A simpler Petri net representing essentially the same coordination without going to the level of initiations and terminations is given in Figure 4.3.3.

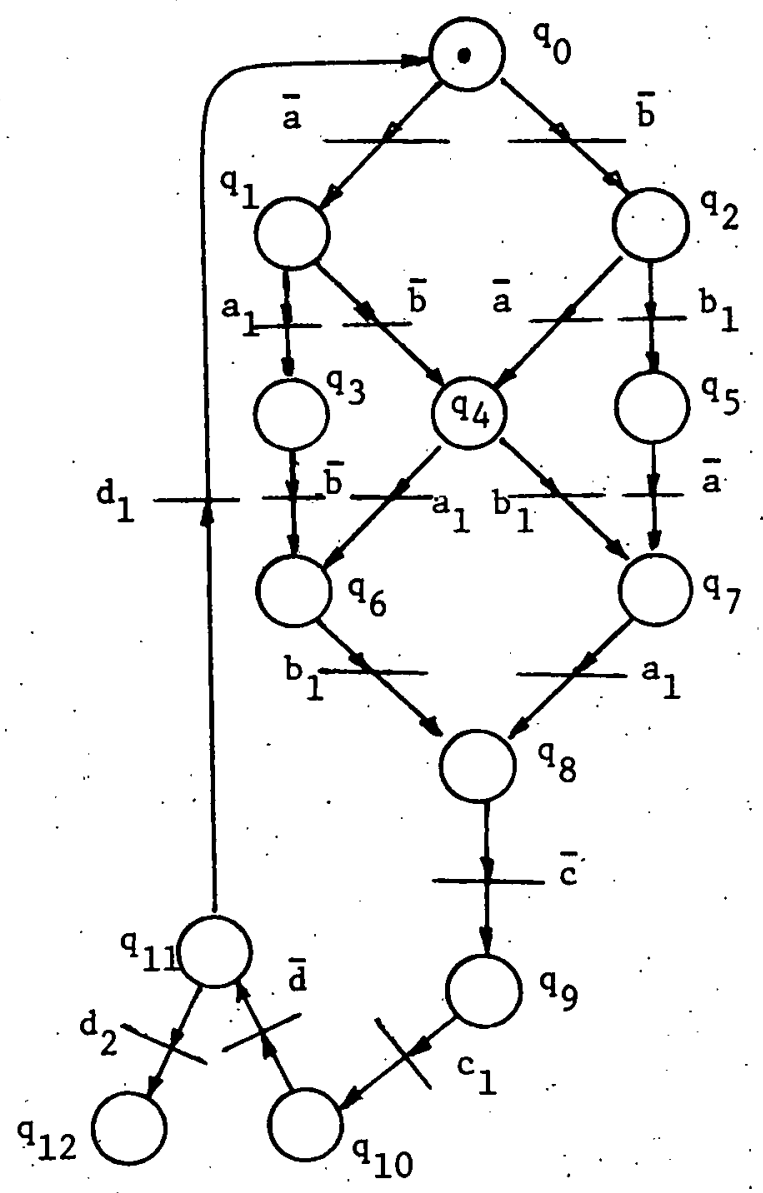

Figure $4: 3.2$ 
Page 51

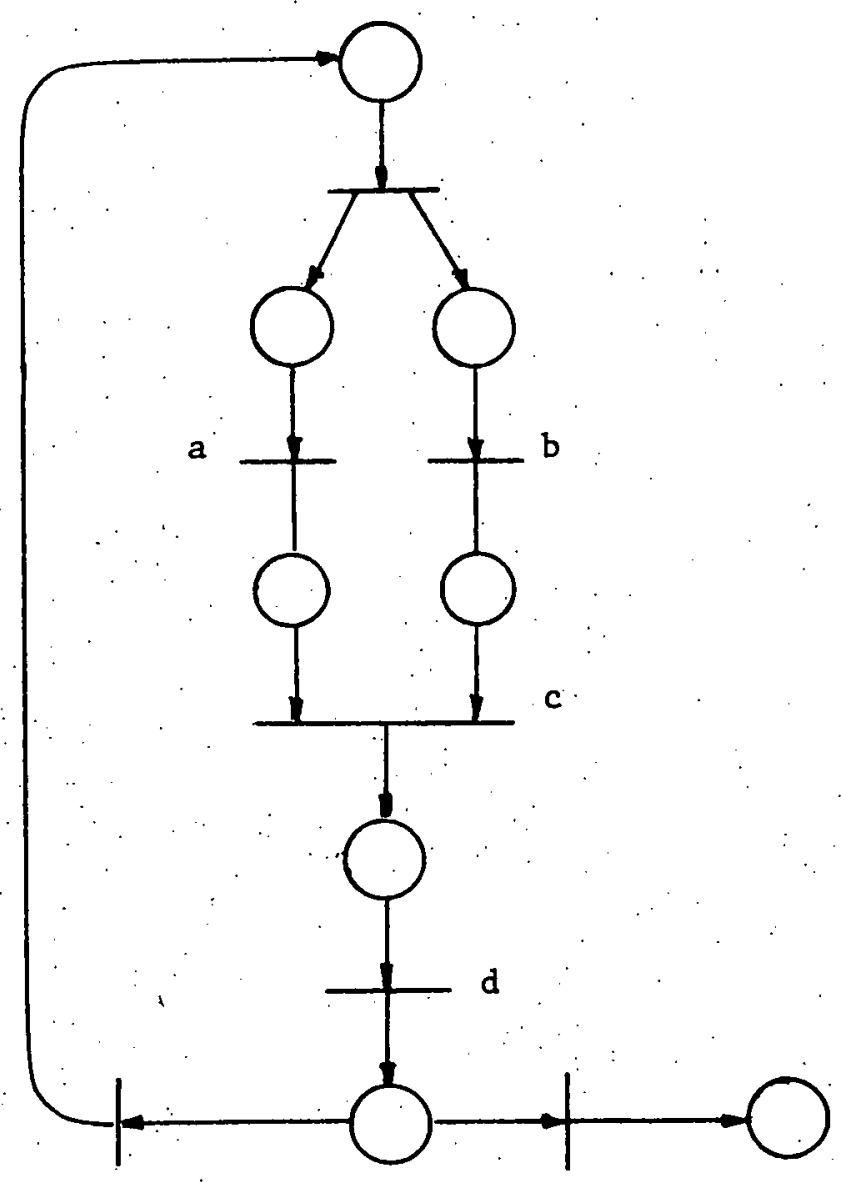

F1gure $4 \cdot 3 \cdot 3$

The nets in Figures 4.3 .2 and 4.3 .3 essentially provide distributed controls. A central control which is a (safe) Petri net can also be constructed, that controls the operations $a, b, c$ and $d$ shown in Figure 4.3.4. $a, b, c$ and $d$ can be viewed as processes not represented by a single transition as in Figure 4.3.3 but split into a set of transitions to represent inftiation, terminations, etc. 
Page 52

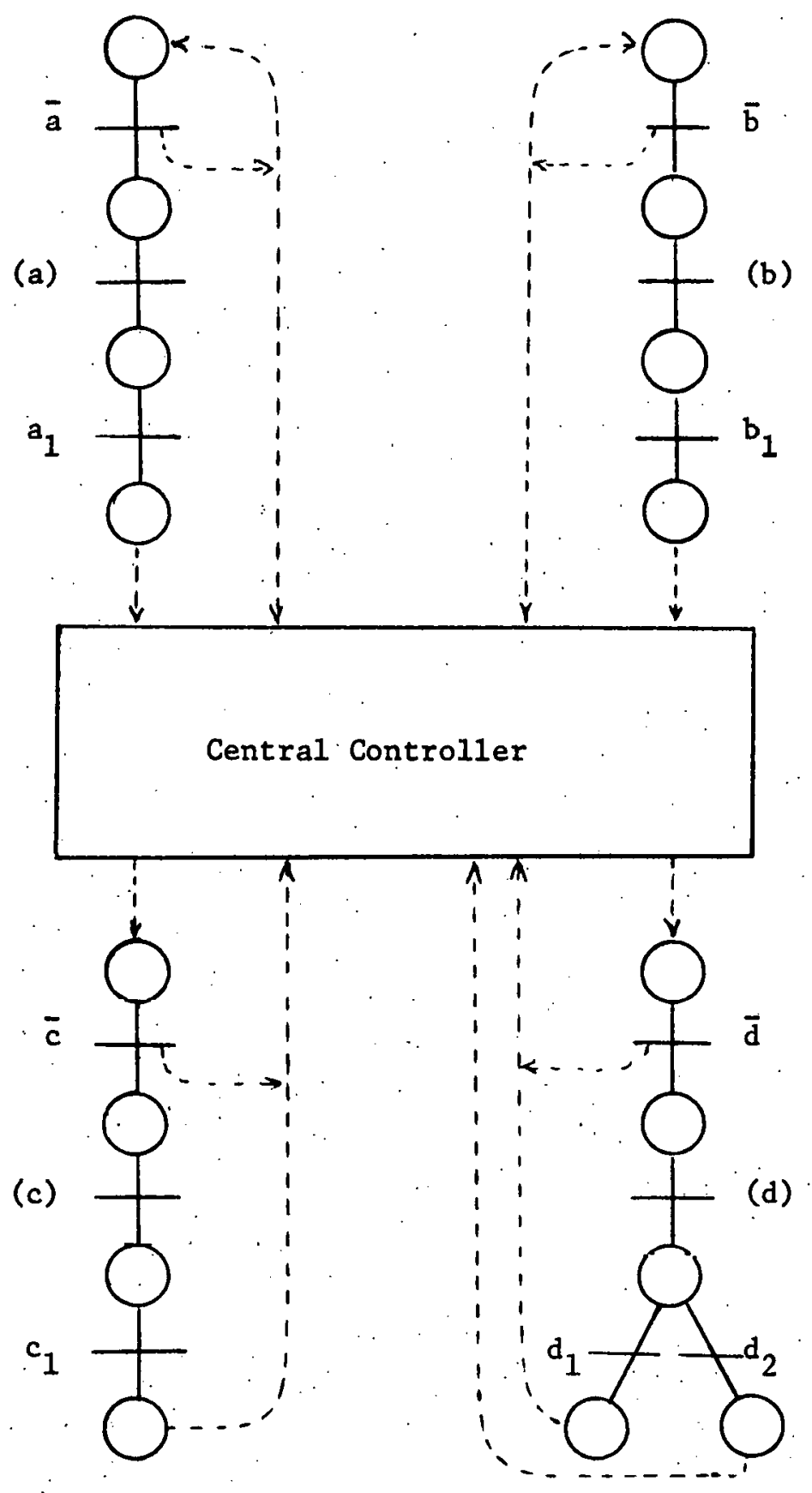

F1gure 4.3.4 
We shall proceed now with another restriction of parallel graph schemas, namely the parallel flow charts. Definition 4.3.3: A parallel flow chart is a three-tuple $F C=\left(M, A, \frac{\pi}{i}\right)$ where

(1) $M$ is a finite set of memory locations.

(2) $\Lambda$ is a finite set of operations and for each a $E A$,

(i) $\mathrm{K}(\mathrm{a})$ is a positive integer specifying the number of outcomes of a

(11) $D(a) \subseteq M$ is the domain of a

(ii1) $R(a) \subseteq M$ is the range of a.

(3) $T=\left(Q, q_{0}, \Sigma, \tau\right)$, the control is specified in terms of a nonnegative integer $p$ and a function $v: \Sigma \rightarrow\{-1,0,1\}^{p}$ as follows :

(i) $Q=N^{P}$ (where $N$ is the set of nonnegative integers) is the set of control states

(ii) $q_{0} \in Q$ is the initial control state

(iii) $\Sigma$ is the union of

$$
\Sigma_{i}=\bigcup_{a \in A}\{\bar{a}\}
$$

the set of initiation symbols and

$$
\Sigma_{t}=\bigcup_{a \in A}\left\{a_{1}, \ldots \ldots, a_{k(a)}\right\}
$$

the set of termination symbols.

(iv) $\tau$. is a partial function, $\tau: Q \times \Sigma \rightarrow$ Q called the transition function. $\tau(q, \sigma)$ is defined if $q+v(\sigma) \geq 0$ and in this case, $\tau(q, \sigma)=q+v(\sigma)$. . The function $v$ has the following constraints: for all a $\varepsilon$ A, v: $\Sigma_{1} \rightarrow\{0, \ldots-1\}^{\mathrm{P}} ; \mathrm{v}: \Sigma_{\mathrm{t}} \rightarrow\{0,1\}^{\mathrm{p}}$; for any two distinct inftiation symbols $\sigma$ and $\sigma^{\prime}$, If $v(\sigma)_{i}=-1$ then $v\left(\sigma^{\prime}\right)_{1}=0$ $\left[x_{i}\right.$ denotes the $i$ th element of vector $\left.x\right]$. 
In this model, the control essentially consists of $p$ counters which always have positive values. The state of the control is then specified at any stage by giving the p-tuple of values of the counters. Each operation $\sigma$ has a p dimensional vector $v(\sigma)$ associated with it: A particular transition (Initiation or termination) is defined if $q+v(\sigma) \geq 0$. Thus terminations are always defined since for $\sigma \in \Sigma_{t}$, $v(\sigma) \geq 0$. Initiation of a particular operation may or may not be defined In some control state. If 1 t 18 defined, that inftiation may take place, leading to a new control state. Once an operation inftiates it must terminate in some finite time. In the framework of Petri nets, an operation a can be viewed as in Figure 4.3.5.

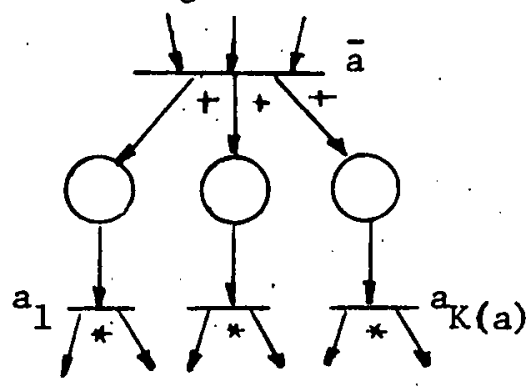

F1gure 4.3 .5

The set $\left\{1 \mid v(\bar{\sigma})_{1}=-1\right\}$ can be vlewed as the set of input counters of operation $\sigma$. The set $\left\{1 \mid v\left(\sigma_{f}\right)_{1}=+1\right\}$ can be viewed as output counters for the termination $\sigma_{j}$ of $\sigma$. Thus, an operation $\sigma$ can initiate only if all the input counters have values greater than zero. When it: inftiates, It decrements the values of the input counters by 1 . Subsequently, $\sigma$ terminates with $\sigma_{j}$ and increments the values of all output counters of $\dot{\sigma}_{j} \cdot$ The restriction on $v$, that for any two $\bar{a}$ and $\bar{b}$, if $v(\bar{a})_{i}=-1$ then $v(\vec{b})_{1}=0$ implies that inftiations do not share input counters. If the operations are viewed as In Figure 4.3.5, the counters as places, and 
of input/output counter relationships are expressed by means of directed arcs, then clearly, a Petri net $\varepsilon \mathrm{TN}_{\mathrm{XORO}}$ results. To investigate the capabilities and limftations of the resulting control, we will not talk about inftiations and terminations separately but assume that each transition represents some process. From this viewpoint, the definition of the control of parallel flow charts corresponds to the definition of the class $\mathrm{TN}_{\mathrm{XORO}}$ with the added constraint that no place has more than one output transition.

Figure 4.3 .6 represents a well behaved trans-labelied net from this latter class. As in Theorem 2.11.4, it can be shown that no well behaved trans-labelled net $\varepsilon$ TN $_{\text {MARK }}$ can represent the same coordination. Thus we conclude that the control of parallel flow charts is more powerful than TN $\mathrm{TARK}_{\text {and }}$ lies somewhere between $\mathrm{TN}_{\text {MARK }}$ and $\mathrm{TN}$.

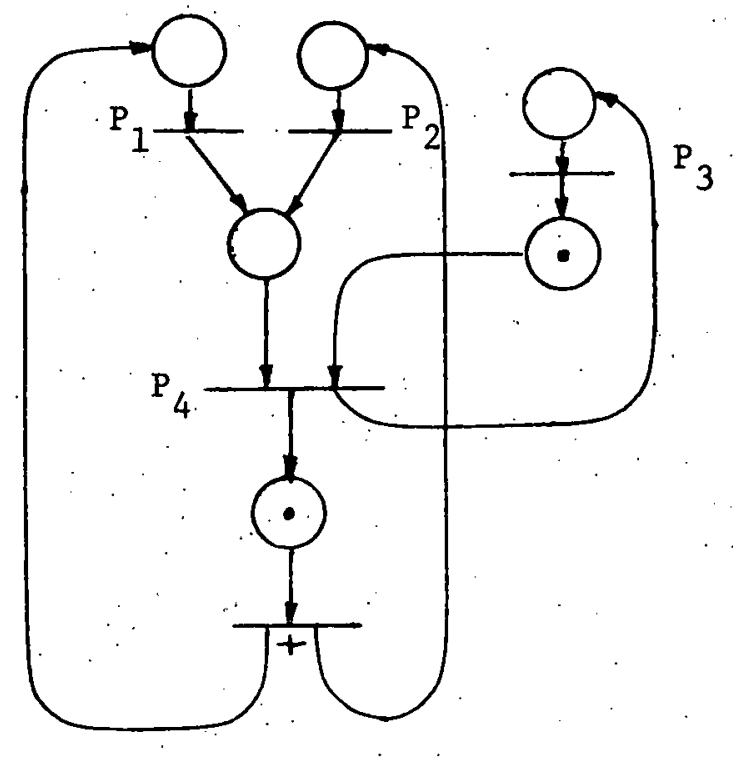

Initial marking ts shown in figure. $F=\left\{\mathbb{M}^{0}\right\}$

Figure 4.3.6 


\subsection{Slutz' Flow Graph Schemata}

The flow graph schemata model, presented and studied by Slutz in 1970 [23], 1s essentially a generallzation of the Karp and Miller parallel flow charts analyzed in the previous section. The model was developed for two reasons. First, it was intended to model more closely certain aspects of parallelism in existing and anticipated systems. Second, it was structured In such a way that propertles like determinacy and equivalence could be investigated more fully.

There are two significant differences between FGS's and parallel flow charts. In Karp and Miller's parallel flow charts, terminations. are always defined. In FGS's terminations are "controlled" just like Initiations. The second significant difference is that a counter can be an Input counter of more than one inftiation or termination.

Definition 4.4.1: A Flow Graph Schema F 1s a three-tuple $F=(M, A, T)$ where

(1) M is a finite set of memory locations.

(2) A is a finite set of operations and for each a $\varepsilon$ :

(1) $K(a)$ is a positive integer specifying the outcomes of a;

(11) $D(a) \subseteq M$ is the domain of a;

(i1i) $R(a) \subseteq M$ is the range of $a$.

(3) $T=\left(Q, q_{0}, \Sigma, \tau\right)$, the control 1s specified in terms of a nonnegative Integer $p$ and a function $v: \Sigma \rightarrow(\{-1\} \text { : } U N)^{p}$ as follows:

(1) $Q=N^{P}$ is the set of control states;

(11) $q_{0} \in Q$ is the initial control state. For each a $\varepsilon$ A there is a coordinate $f_{a}$ such that $\left(q_{0}\right)_{a}=0$;

(iii) $\Sigma=\bigcup_{a \in A}\left\{\bar{a}, a_{1}, \ldots \ldots, a_{K(a)}\right\}$ is the alphabet; 
(1v) $\tau$ is a partlal function, $\tau:: Q \times \Sigma \rightarrow Q$ is called the transition function. $\tau(q, \sigma) 18$ defined if $q+v(\sigma) \geq 0$ in which case $\tau(q, \sigma)=q+v(\sigma)$. The function $v$ has the following constraints:

(a) $\left(v\left(a_{1}\right)\right)_{k}=-1 \Rightarrow\left(v\left(a_{j}\right)\right)_{k}--1,1 \leq 1, f \leq K(a)$

(b) for all b $\varepsilon \sum$ where $b \neq \bar{a}, a_{1}, \ldots \ldots, a_{k(a)},(v(\bar{a}))_{1_{a}}=1$, $\left(v\left(a_{1}\right)\right)_{f_{a}}=-1$ and $(v(b))_{J_{a}}=0$.

Restriction (a) on $v$ is the requirement that all terminations of an operation have the same set of input counters. Restriction (b) Is the requirement that a counter $f_{a}$ exist (which is inftially empty) that is an output counter only for $\bar{a}$ and an input counter only for terminations of a.

Each operation a, In the Petri net framework, can be viewed as in F1gure 4.4 .1

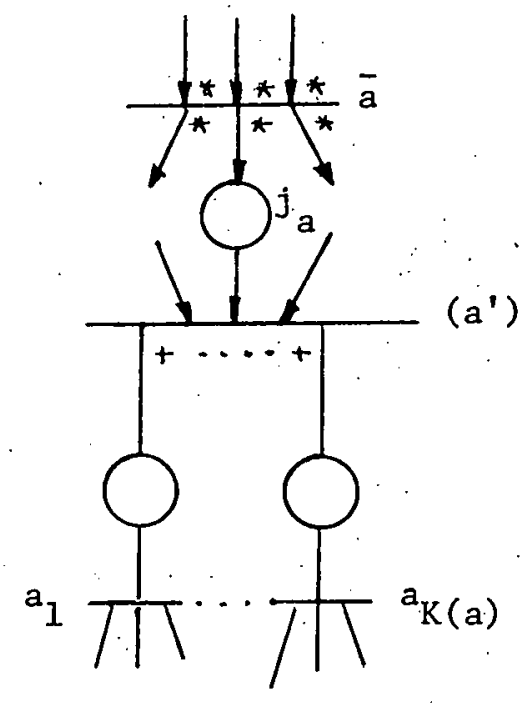

\section{Figure 4.4 .1}

The counters can be viewed as places. The input places of $\bar{a}$ consist of the set $\left\{1 \mid(v(\bar{a}))_{1}=-1\right\}$ and the output places form the set $\left\{1 \mid(v(\bar{a}))_{1}>0\right\}$. The set of Input places of $a^{\prime}$ is $\left\{1 \mid\left(v\left(a_{1}\right)\right)_{1}=-1\right\}$; $a^{\prime}$ has $K(a)$ output places and the output logic is EOR. The set of output 
places of $a_{j}$ is $\left\{1 \mid\left(v\left(a_{f}\right)\right)_{1} \geq 0\right\}, 1 \leq j \leq K(a)$. Thus, after inftiation of a, termination is controlled by $a^{\prime}$. Firing of $a^{\prime}$ arbitrarily enables one of the termination symbols. Each of the $\sigma^{\prime}$ transitions can be replaced by a net belonging to $\mathrm{TN}_{\mathrm{PLEX}}$ in a manner simflar to the replacement procedure in the proof of Theorem 2.11.6. The resulting net is a member of $\mathrm{TN}_{\mathrm{PLEX}}$ and represents the same control as the original FGS. Once again, if the transitions represent arbitrary processes rather than Inftiations and terminations it is easy to see that the definition of the control in an FGS corresponds exactly to the class TN PLEX'

\subsection{Lucont's Computational Schemata}

Th1s is another graph model, presented in 1968 [16] with the intent to describe finite, discrete information systems. The model consists of a set of operators standing in fixed relation to one another with respect to paths of communication. The connecting lines may carry data, operator sequencing and control information, timing sequences, and so on. A partial function is associated with each operator defining when and how each operator can process information appearing on the cormunication lines constituting its inputs. The results of processing are distributed to the operator's output lines (which may include some of the input ines). Because the timing of this processing is arbitrary, operators act asynchronously with respect to each other. More formally:

Definition 4.5.1: A computational schema is an ordered 5-tuple $S=(A, L, T, \delta, \rho)$ where

(1) $A=\left\{a_{1}, \ldots \ldots, a_{n}\right\}$ is a finite set of operators;

(2) $L=\left\{\ell_{1}, \ldots . .,, \ell_{m}\right\}$ is a finite set of links; 
(3) $T \subseteq L$ is a set of links spectfying the schema Interface or terminal set,

(4) $\delta: A \rightarrow L^{d}$ is a function spec1fying the links constituting each operators linput set,

(5) $P: A \rightarrow L^{r}$ is a function specifying the links constituting each operators output set.

In addition, a discrete value can be associated with each 1ink. No restrictions are placed on the nature of this value. $V\left(l_{1}\right)$ denotes the set containing every value that could be associated with link $\ell_{1}$. Each operator a $\varepsilon A$ has a vector of input links:

$$
\delta(a)=\left(\delta^{1}(a), \ldots \ldots, \delta^{d}(a)\right), \delta^{j}(a) \in L
$$

and a vector of output. IInks :

$$
\left.\rho(a)=\rho^{1}(a), \ldots \ldots, \rho^{r}(a)\right), \rho^{j}(a) \in L \text {. }
$$

The local configuration $r(a)$ defined for operator a is the vector:

$$
(a)=\left(\gamma^{1}(a), \ldots \ldots, \gamma^{d}(a)\right)
$$

where each $\gamma^{1}(a)$ is the value assoclated with link $\delta^{1}(a)$. A partial function $f(a)$ is defined for each operator a $\varepsilon$ A. Thus:

$$
\mathbf{f}_{\mathbf{a}}: \mathrm{D}_{\mathbf{a}} \rightarrow \mathrm{R}_{\mathbf{a}}
$$

where

$$
\begin{aligned}
& D_{a} \subset \underset{i=1}{X}\left[V\left(\delta^{1}(a)\right)\right] \\
& R_{a} \subseteq{\underset{1=1}{X}}_{X}^{r}\left[V\left(p^{1}(a)\right) \cup\{(-)\}\right]
\end{aligned}
$$

If operator a has local configuration $\gamma(a)$ and if $\gamma(a) \in D_{a}$ then operator a is applicable. When an operator a applies, it replaces the values associated with the IInks of its output set according to $f_{a}$. ( (-) Indicates that the value of the assoclated output link is not to be changed). 
A schema defined in the above manner operates as follows. Inttially, each link $l_{1}$ is associated with a value belonging to $V\left(l_{1}\right)$. The linkvalue associations at any stage define a local configuration for each operator a $\varepsilon A$. An operator may or may not be applicable in a given local configuration. If it 18 , and if it applies, it redefines the values assoclated with some links. The conditions for applicabllity being local, the operators proceed asynchronously.

With the above definition of a schema we have two alternatives. The schema can be considered to be an interpreted model, where the function associated with each operator a can be thought of as specifying the behavior of a. In this case, the schema can clearly be considered to represent a computation. The second alternative is to consider the function $f_{a}$ as merely determining when a particular operation a can be Intiated and the effect it has on its environment on terminating. Here each operator can be thought of as representing a particular process (that 18 activated when a initiates and on whose completion, a terminates) that affects entities completely distinct from anything in the schema. Nelther the effects nor the entities affected are known. Looking at the schema from this perspective ylelds an uninterpreted model. It is this uninterpreted model, representing a controlling agent that will be analyzed here. In the second half of his thesis Lucont vlews a schema as composed of two parts called the Interpretation schema (I-schema) and the Control schema (C-schema). Such a schema is called a structural schema. The I-schema performs the computations and the C-schema determines when operators in the I-schema are enabled. A connection can exist between an I-operator a and a C-operator b through a comon link, 
control link. (See Figure 4.5.1). In a restricted form each operation In either schema can have at most one input and one output control link.

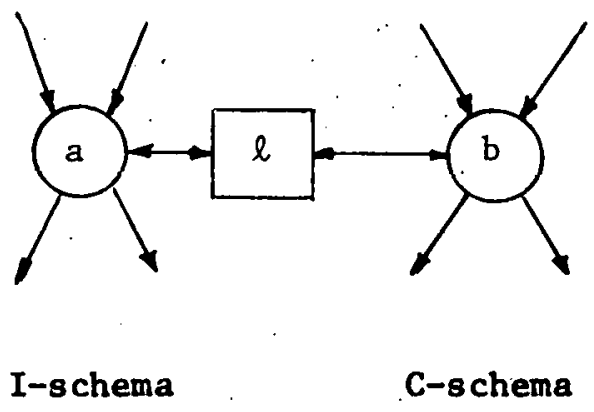

F1gure 4.5.1

Before an operator in the I-schema is eligible for initiation the common link must have a value 0 . When the I-schema operator is eligible for Initiation the value of the control link is set to one by the $c$ schema operator. When the I-schema operator terminates its execution, It sets the value of the control varlable to 2 . It should be noted that the uninterpreted schema we are considering does not correspond exactly to a C-schema.

To proceed with the analysis of the general computational schema viewed as a controlling agent, in the definition of the schema, no restrictions are placed either on the values that may be associated with links or on the function $f_{a}$ associated with each operator a $\varepsilon A$. In this general form, the specification corresponds to a controlling agent as powerful as the Turing machine central controller [3], and hence as powerful as the extended Petri nets. (Whether such a control is useful other than for theoretical purposes and for studying questions regarding decidability is a different matter and will be discussed elsewhere.) One restricted class of computational schemas is the class of finite state computational schemas. 
Page 62

Definition 4.5.2: A finite state computational schema, $S$, is a schema in which all values that can be assoclated with a link are required to be members of some finite set $v_{8}, 1 . e ., v\left(l_{1}\right) \subseteq v_{8}$ for all 1 .

As Lucon1 shows, a finite state transition graph $G(S)$ can be defined for any finite state schema $S$ as follows:

(1) A node in $G(S)$ corresponds to a state of $S$ where a state is defined as a particular set of link value associations. Since each link can take on only a finite number of values, there are only a finite number of states.

(11) A directed arc is drawn from node 1 to node $j$ and labelled a if operator a is applicable in the state corresponding to node 1 and if application of a in this state leads to the state corresponding to node $f$.

Clearly, G(S) represents the same control as S. Similarly, for every finite state machine (representing a control) a finite state schema can be given that represents the same control. We can thus conclude that finite state schemas = $\mathrm{TN}_{\mathrm{SAFE}}$.

The other restricted schemas studied by Lucont are well-formed schemas. A schema is well-formed if it is conflict-free and transformat1onal-lossless.A schema is conflict-free if for any pair of operators a and $b$ that can become applicable simultaneously, elther (1) a and b have no output links in common, or (11) $a$ and $b$ do not alter the value of a common output 1 ink, or (111) both a and b indicate Identical value changes to all common output links. A schema is transformational-1ossless if for any pair of operators $a$ and $b$ that can become applicable simultaneously efther (1) a and b do not communicate or (11) the application of neither can affect the value changes about to be made by the other. 
Thus, in well-formed schemas, once an operation becomes applicable, it continues to remain applicable. A very formal analysis has not been conducted but the above fact seems to Indicate that there exist problems where the desired control can be represented by ordinary Petri nets but not by well-formed schemas. Whether the reverse is true is not known. Clearly, the coordination represented by the net in Figure 4.3 .6 can be represented by a well-formed schema. Thus, well-formed schemas are more powerful than marked graphs.

\subsection{Rodriguez' Graph Model of Computation}

This model was proposed and studied by Rodriguez in 1969 [22]. The underlying control is bastcally a finite, well-formed schema." In Section 4:5 we considered a schema to correspond to a controlling agent. Each activation of an operator was considered to correspond to the inftiation of some one process. An operation was activated when the vector of values of the input links belonged to a specific set. On termination values were assigned to the output links depending on the values of the Input links at the time of activation. Irrespective of the conditions under which a node was activated, the same process was initiated. In the Rodriguez model, the process initiated will depend, in general, on the value of the input links at the time of activation. Thus the underlying control here corresponds more closely to a C-schema.

The model is composed of nodes (which correspond to computational elements) and links (which correspond to storage-transmission elements). There are two types of links: a data link and a control link. Associated with both data and control links is a quantity called link status. At any given time the link status of a link assumes precisely one of the 
Page 64

four possible values $-1,0,1,2$. These values are called disabled, 1dle, enabled and blocked respectively. In addition, data links can carry data. There are seven types of nodes. Nodes have specific points of attachment called connectors. Connectors are either input or output connectors. The activation of a node is determined by the Iink status of the links attached to the node. A node is in an active configuration when the statuses of 1 ts connectors 18 such that the node becomes active. The occurrence of an active configuration initiates a transition of arbitrary time length. On completion of the transition, the status of each connector (and perhaps the data contents of the attached data 11nks as well) are changed in a way specified by the transition table for that type of node.

The program graph nodes and their transition tables are given in Figure 4.6.1. Thus, for the function operator one transition is

$110 \rightarrow 001 \quad \$ 3+f(\$ 1, \$ 2)$

This transition Indicates that if the statuses of links 1,2 and 3 are $1,1,0$ respectively, then the data contents of connector 3 are replaced by the result of applying $f$ to the data contents of connectors 2 and 1 , and the statuses of links 1,2 and 3 are given the values 0,0, and 1 respectively. For an in-depth explanation of the different nodes, the reader is referred to Rodriguez' thesis.

A program graph is a finite set of input terminals, output terminals and nodes Interconnected by data links and control links according to the following rules:

(1) The root of a data link must be attached to an input terminal or to a data output connector and its t1p must be attached to 


\section{Operator}

Data operator

Function

Identity

\section{Control operator}

And

$\underline{\text { Or }}$

Selector

Junction
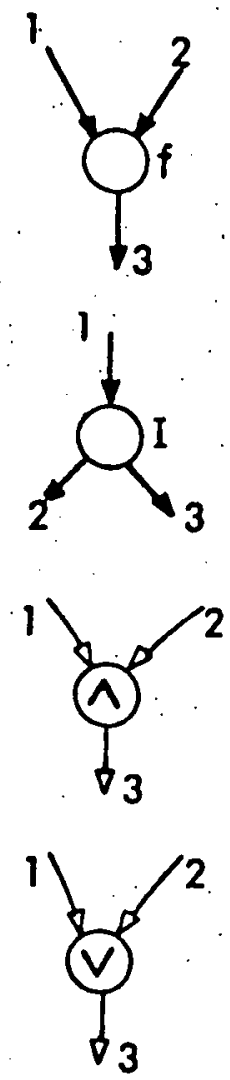

$110 \rightarrow 001 \quad \$ 3-1(\$ 1, \$ 2)$

$1-1 \quad 0 \rightarrow 00-1$

$-110 \rightarrow 0-1$

$-1-1 \quad 0 \rightarrow 0.0-1$

$100 \rightarrow 011$

$-100-0-1-1$

\section{$\$ 2, \$ 3-\$ 1$}

$110 \rightarrow 001$

- $1-10 \rightarrow 00-1$.

$\begin{array}{llllll}-1 & 0 & 0 & 0 & -1\end{array}$

$-1-1 \quad 0 \rightarrow 00-1$

$11.0 \rightarrow 001$

$1-10 \rightarrow 001$

$\begin{array}{cccccc}-1 & 1 & 0 & \rightarrow & 0 & 0\end{array}$

$-1-10 \rightarrow 00-1$

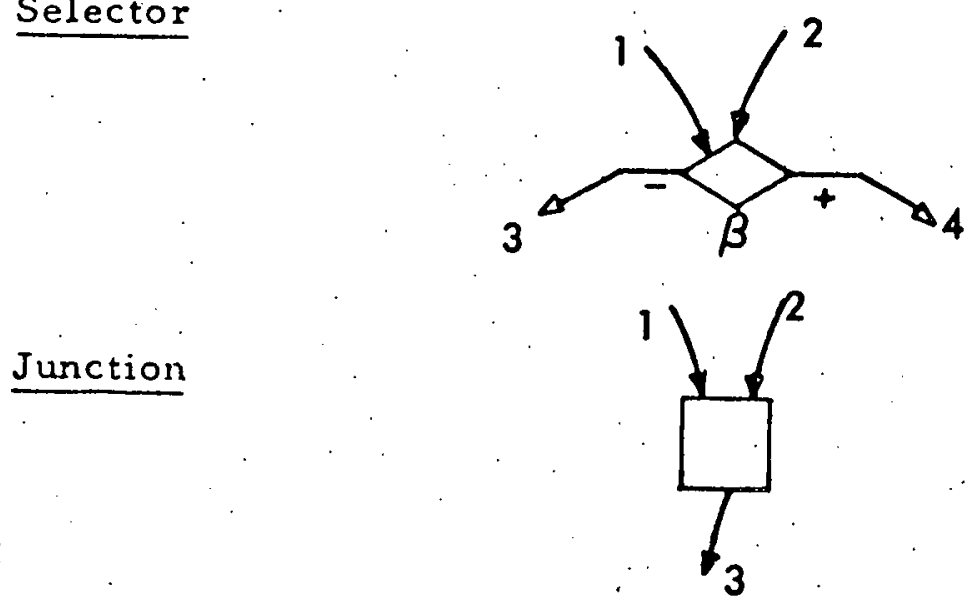

$11000 \rightarrow\left\{\begin{array}{ccccc}0 & 0 & -1 & 1 & \text { if } \beta(\$ 1, \$ 2)=\text { true } \\ 0 & 0 & 1 & -1 & \text { if } \beta(\$ 1, \$ 2)=\text { false }\end{array}\right.$

$1-100 \div 00-1-1$

$-1.100 \rightarrow 00-1-1$

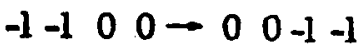

$1-10-001 \quad \$ 3-\$ 1$

$-1: 10 \rightarrow 001 \quad \$ 3-\$ 2$

$-1-10 \rightarrow 00-1$

Figure 4.6.1 
Page 66

\section{Loop Junction}

\section{Loop Output}

Input Terminal

Output Terminal
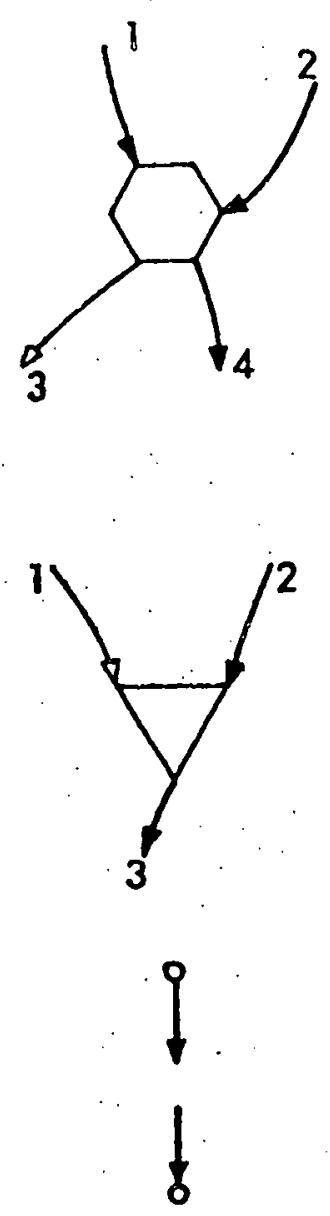

$$
\begin{aligned}
& 1-0 \\
& -1-0
\end{aligned}
$$

Figure 4.6.1 (cont . $\left.^{\prime}\right)$ 
an output terminal or to a data Input connector.

(2) The root of a control link must be attached to a control output connector and 1ts tip must be attached to a control input connector.

(3) Every Input connector of a node must be attached to a control Input connector.

All links are in the 1dle (0) state initially. At some time to, all Input terminals are placed in an enabled state simultaneously and execution begins. Figure 4.6.2 gives an example of a program graph.

The underlying controlling agent is now obtained as follows: No distinction is made between data links and control links.

All links are control links. Let $\left\{l_{1}, \ldots \ldots l_{n}\right\}$ denote the set of nodes in any program graph. Each node $l_{1}$ has an asosclated transition table $t_{i}$ containing a set of transitions $\left\{t_{1_{1}}, \ldots t_{1_{k}(1)}\right\}$. The $t_{1_{j}}$ 's are as shown in Figure 4.6.1 except that in each transition $t_{1_{j}}$, if there Is a data transformation specified, it is replaced by a symbol $\sigma_{i j}$. Thus If $l_{1}$ is a function node, the transition $t_{1_{1}}=110 \leftarrow 001 \$ 3 \leftarrow f(\$ 1, \$ 2)$

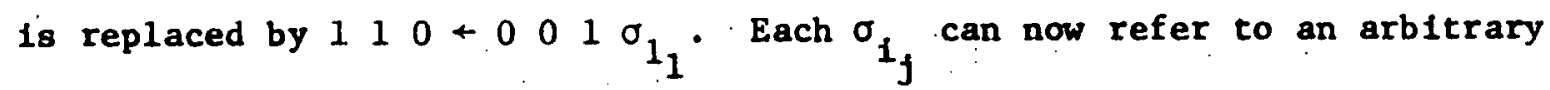
process. For each selector node, the transition

$\begin{array}{llll}1 & 0 & 0\end{array} \rightarrow\left\{\begin{array}{llllll}0 & 0 & -1 & 1 & \text { if } & \beta(\$ 1, \$ 2)=\text { True } \\ 0 & 0 & 1 & -1 & \text { if } \beta(\$ 1, \$ 2)=\text { False }\end{array}\right.$ 18 replaced by two transitions

$1100 \rightarrow 00-11$

$1100+001-1$

Thus, if the configuration of the selector is 1100 , either one of the two transitions could take place arbitrarily. If a function $A$ is defined 
$\operatorname{sart}(\sin (x \mid 2)+\cos (1-\tan (y) \uparrow 2))$

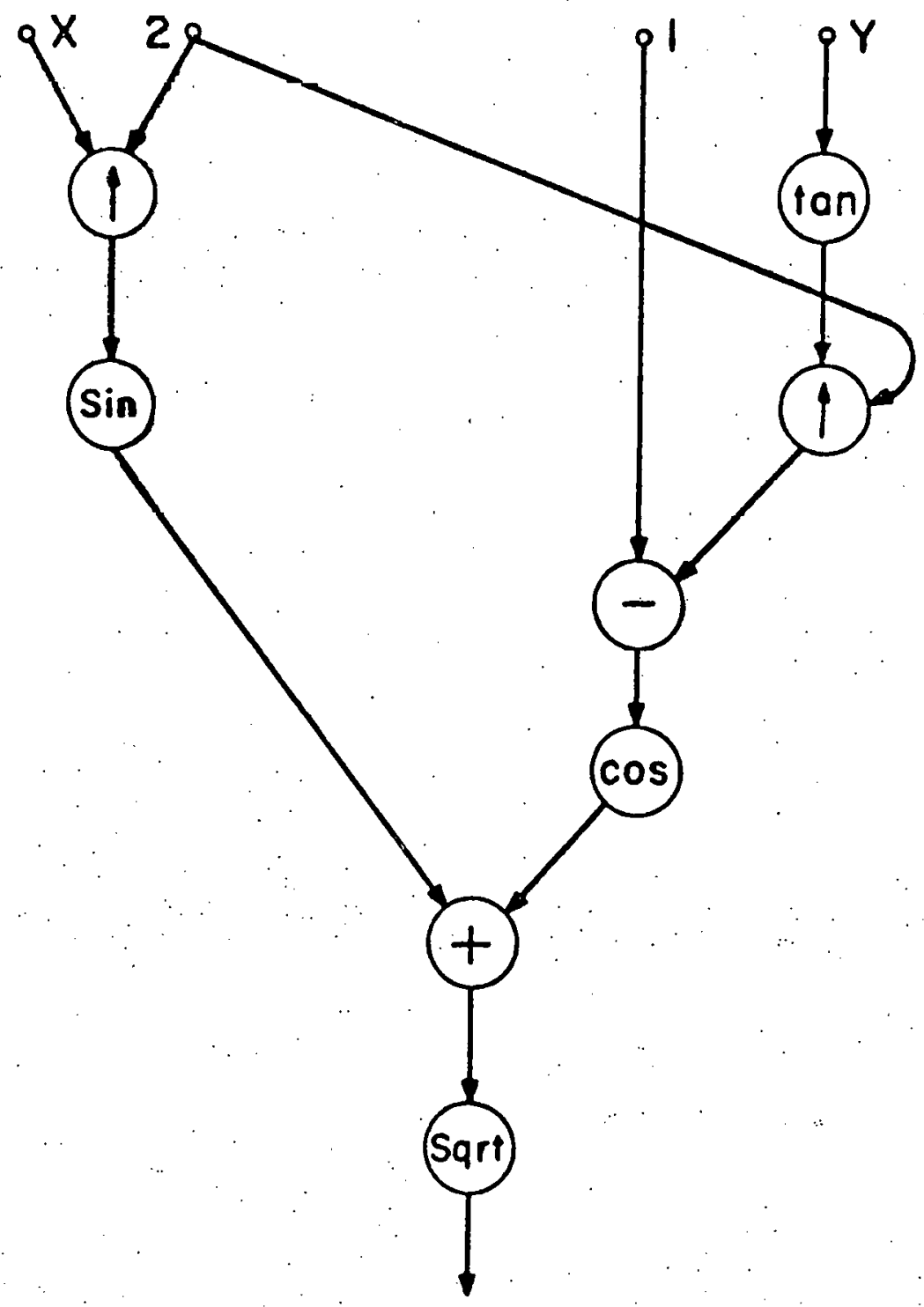

Figure 4.6 .2 
for a graph $G$ such that $A$ maps the set of $\sigma_{f_{j}}$ 's (say $Q$ ) onto a set $\left\{P_{1}, \ldots \ldots, P_{k}\right\}$ where $k \leq \# \Omega$ then $G$ and $A$ represent a coordination of the processes $\left\{P_{1}, \ldots \ldots, P_{n}\right\}$ rather than a computation.

Let a program graph $G$ have $p$ links. Since each link can take on at most 4 status values, the p-tuple of status values of the link can be considered to constitute a "system state" of G. Clearly, G has a finite number of system states (say $\left.\left\{q_{1}, \ldots \ldots, q_{8}\right\}\right)$. A finfte state transition diagram, G', corresponding to $G$, can be constructed as follows:

$G^{\prime}$ has $S$ nodes, $q_{1}, \ldots \ldots, q_{8}$, each corresponding to a unique system state of $G$. An arc is drawn between $q_{i}$ and $q_{j}$ and labelled $x_{k_{m}}$ if node $l_{k}$ of $G$ is in an active configuration in state $q_{i}$ and the mth transition of table $t_{k}$ causes the state to change from $q_{i}$ to $q_{j}$. When all the arcs are drawn each label $x_{k_{m}}$ is replaced by $P$ if $A\left(\sigma_{k_{m}}\right)=P$ and is deleted. If $A\left(\sigma_{k_{m}}\right)$ is undefined. Clearly, G' represents the same coordination as G. Since $\mathrm{TN}_{\mathrm{SAFE}}$ can represent any coordination that can be represented by a finite state transition diagram, we conclude that the underlying control in Rodriguez' model $\subseteq \mathrm{TN}_{\mathrm{S} \Lambda F E} \cdot$

\subsection{Adam's Model}

Adam's model presented In 1970 [1]. is an interpreted model, the motivation once again being the study of parallel computations. The model 1s fairly complex and we shall not attempt to formally define it. The main features will be explained and the underlying controlling agent extracted.

The model consists of a directed graph with the nodes representing computation steps and the arcs, FIFO queues of data. In the Adam model each node can be either a primitive node or a procedure where the latter 
Is also defined as an interpreted graph. For out purpose we will consider each node to be a primltive node representing as usual, some arbitrary process rather than a particular computation.

The possible data types are defined separately by means of a context free grammar. Each edge $e$ is of a particular data type $d$ and the parameter d Identifies the structure of the elements found on the queue assoclated with e. Primltive nodes are of two types, $r$ or 8 . $r$ nodes are computational and $s$ nodes, both computational and $\log 1 \mathrm{cal}$. The inpurs of each s node carry status values. The status of a particular input edge can be locked (L) or unlocked (U). With each $r$ node 18 associated a function $f$ and with each $s$ node, two functions $f$ and $g$. The $f$ function receives an agrument from each input edge that is unlocked. $f$ may produce output on only some or possibly none of the output edges. More than one element may be placed on an edge as output. The $g$ function associated with an 8 node allows the status settings of that node to be changed as a function of the current settings and the input arguments of the node. For a given ordered set of Inputs, both $f$ and $g$ have uniquely defined outputs.

Informally, the program graph executes as follows: The initial data for the program 18 placed on the appropriate edges. During the execution of the graph program, a node in the graph may be executed whenever there 18 at least one element of data on each of its unlocked input edges. When the node executes, it removes one element of data from each unlocked input, performs the Indicated computation, and places the results on the appropriate edges leading out from the node. For the snodes, the status settings may also be changed. When no further nodes 
can be executed, the computation is complete, and the results will be left on the nodes designated as the output set of $G$.

For simplicity, let us assume that no activation of a node places more than one result on any output arc. In the Petri net framework, each edge in a graph $G$ can be replaced by a place. Each data element is a marker. Each node corresponds to a net, as will be explained. For any node, let $I=\left\{e_{1}, e_{2}, \ldots \ldots e_{k}\right\}$ be the set of input edges and $0=$ $\left\{e_{k+1}, \ldots, \ldots, e_{k+m}\right\}$ the set of output edges. Then an r-node can be represented as shown in Figure 4.7.1.

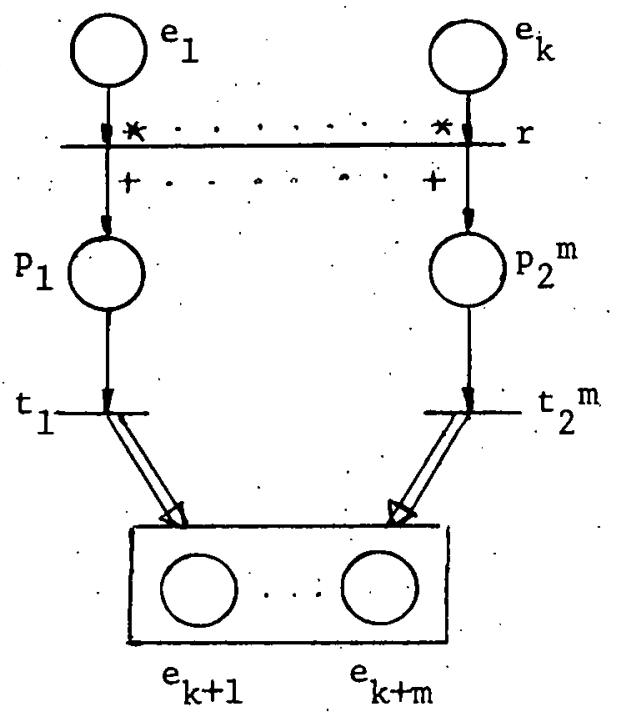

Figure 4.7.1

Each $t_{1}$ has as its output places a particular subset of $2^{0}$. The transition $r$ has an exclusive $-O R$ output because, depending on $f$ and the arguments on the input edges of $r$, any subset of the output edges would receive data. The s-node 18 represented by a more complicated network, because of the associated status values. The net to replace the s-node 18 given 
in F1gure 4.7.2. Each place $q_{1}, 1 \leq 1 \leq 2^{k}$ represents a particular assignment of status values to the edges $e_{1}, \ldots, e_{k}, 1 . e$, , represents a particular subset $K_{1}$ of $2^{I}$. Each transition $S_{1}$ has as inputs, place $q_{1}$ and the set of places $k_{1}$. Each of $s_{1}, s_{2}, \ldots, s_{2} k$ represents node 8. Firing of one of these activates transition $t$ which selects the

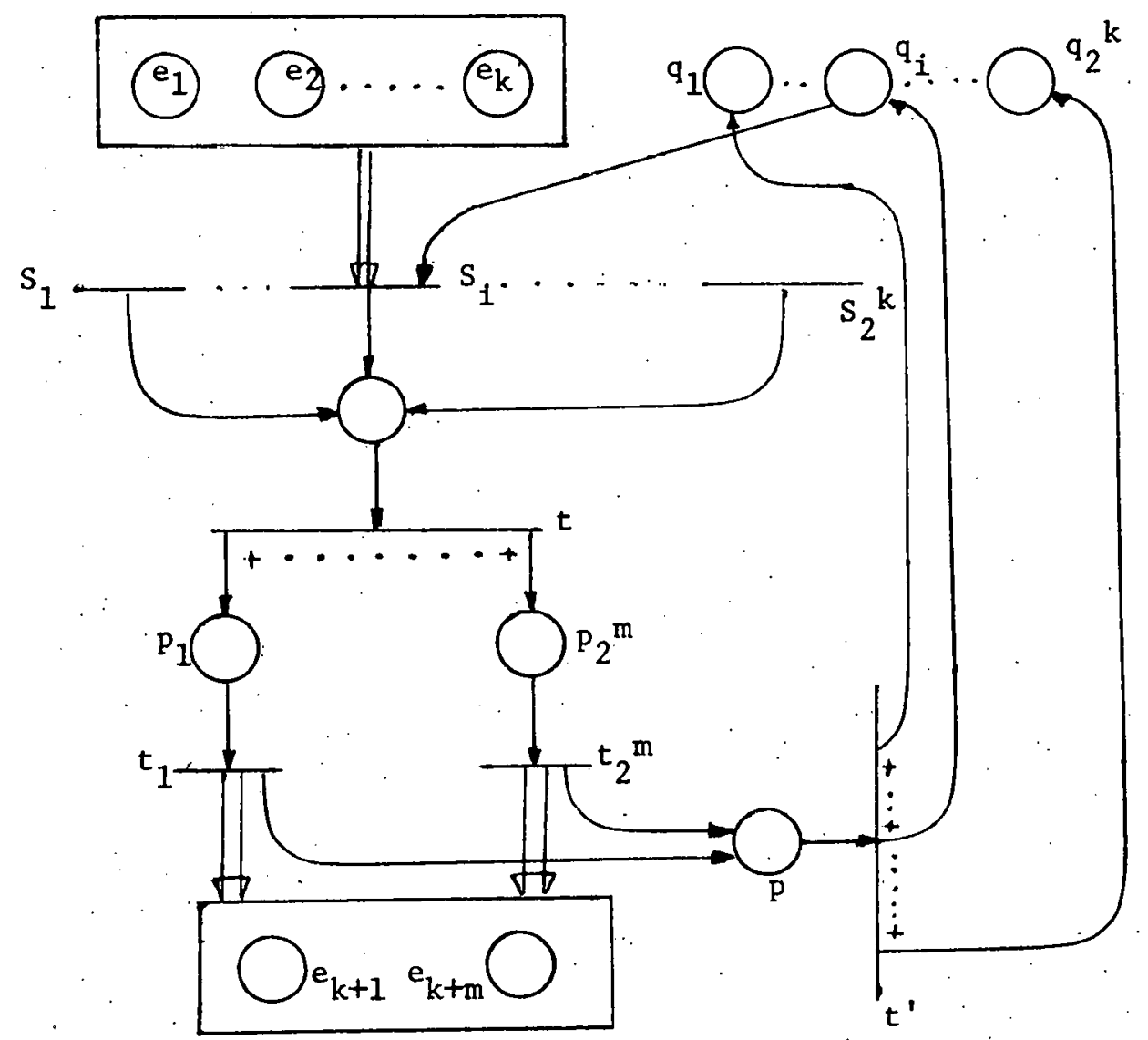

Figure 4.7 .2 
subset of $2^{0}$ to recelve markers. When markers have been placed in the appropriate output places, $t$ ' is also activated. $t$ ' arbitrarily selects the next set of status values. We will assume that given a graph model, the underlying controlling agent is obtained by the above two replacement procedures. The resulting control is then clearly a subset of $\mathrm{INN}_{\mathrm{XORO}}$, where once a process has been enabled, it cannot be disabled by any other process.

\subsection{Peterson's P-nets}

This model was studied by Peterson in 1973 [19], who viewed it as the control structure of a model of parallel computation. The p-net is essentialiy an ordinary Petri net where multiple arcs are allowed between places and transitions and between transitions and places.

The p-net is a directed bi-partite graph with two types of nodes, places (represented by circles) and transitions (represented by bars). Arcs are directed from one type of node to the other. Arcs directed into a node define its inputs whtle arcs directed out of a node define its outputs. Each transition has an operation or label associated with 1 t. A start place and a set of final places are defined for each p-net. Figure 4.8.1 gives an example of a p-net.

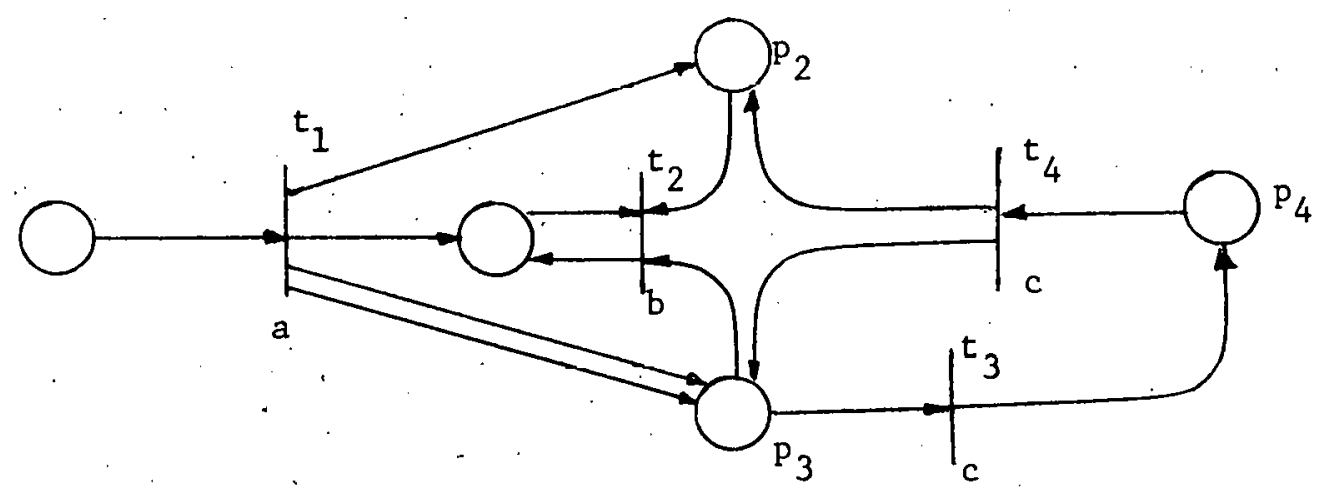

F1gure 4.8 .1 
The simulation rules are the ame as for ordinary Petri nets except that multiple arcs require multiple tokens. The labels assoclated with the arcs correspond to a particular trans-labelling. Corresponding to Intial and final places, Initial and final markings can be defined. Multiple arcs, as between $t_{1}$ and $p_{3}$; can be replaced by a single arc with a numeral indicating the number of markers required. If instead of a start place and final places, inftial and final markings are defined, then clearly, p-nets $=\mathrm{TN}_{\mathrm{PLEX}}$.

\subsection{Brer's Switch Petr1 nets}

Th1s modifled form of Petr1 nets was introduced by Baer in 1973 [7]. In addition to ordinary transitions and places Baer allows the following: Disfunctive Input transitions (Figure 4.9.1), disjunctive output transitions (F1gure 4.9.1), swltches (F1gure 4.9.2) and token absorbers (FIgure 4.9.3).

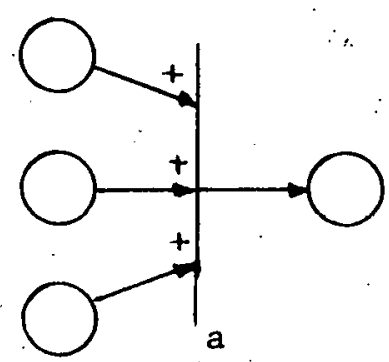

Input D1s junctive Log1C. Only one of the Input places can be full. Then a can fire.

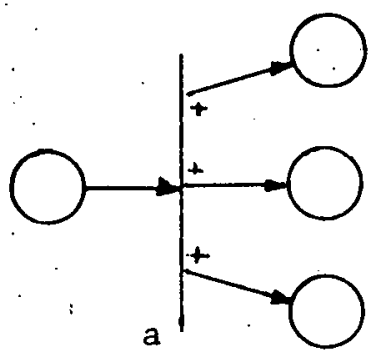

Output Disfunctive Logic After firing of a, only one of the output places will recelve a token.

F1gure 4.9.1
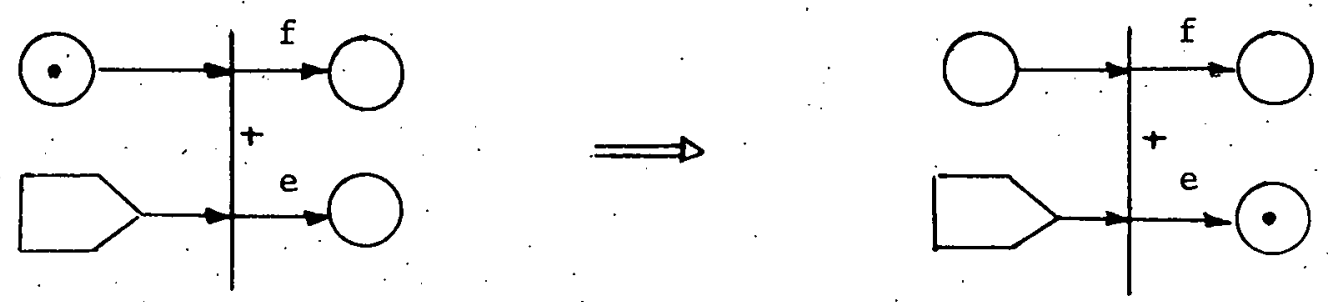

(a) Empty Swtch 


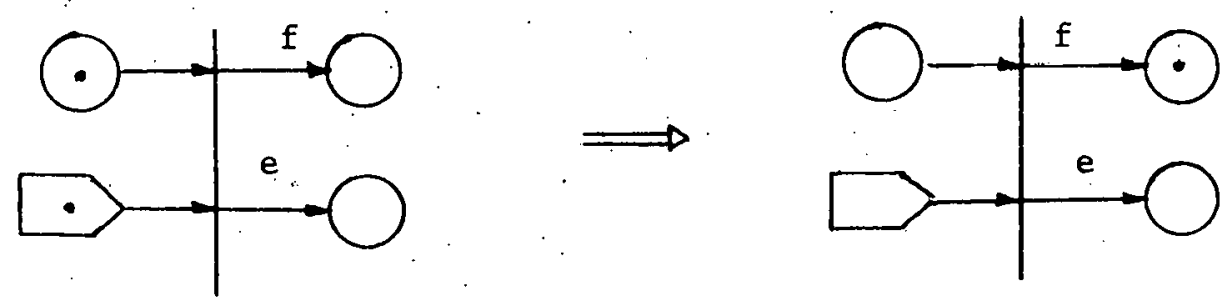

(b) Full Switch

Figure 4.9 .2

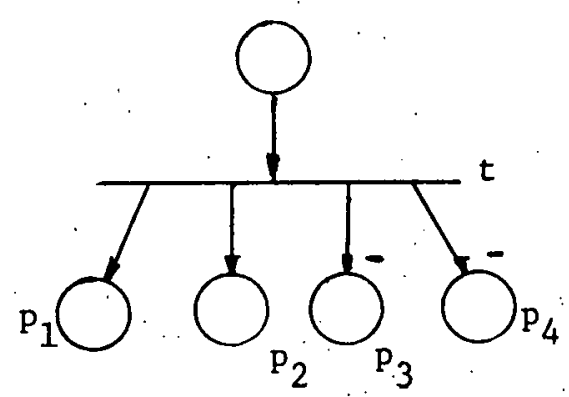

token absorber

When $t$ f1res, it places one marker

in each of $p_{1}$ and $p_{2}$ and removes

all markers ${ }^{1}$ from $\mathrm{p}_{3}$ and $\mathrm{p}_{4}$.

Figure 4.9.3

Formally :

Definition 4.9.1: The switch Petri net is a triple $P=(X, A, C)$ where:

$X=P U T$ is a finite set of vertices with

$P=\left\{p_{1}, \ldots \ldots, p_{n}\right\}$ being a fintte set of places;

$T=\left\{t_{1}, \ldots \ldots, t_{m}\right\}$ beling a finite set of transitions;

S : a possibly empty proper subset of $P$ is a set of switches;

$A=I U O U N$ is a finite set of arcs with

$I=\left\{\left(p_{i}, t_{j}\right) \mid p_{i} \varepsilon \cdot P, t_{j} \varepsilon T\right\}$ being the input arc set and $p_{1}$ being an input place to $t_{j}$; 
$0=\left\{\left(t_{1}, p_{j}\right) \mid t_{1} \in T\right.$ and $\left.p_{j} \in P\right\}$ being the output arc set and $p_{f}$ belng an output place to $\mathrm{T}_{1}$.

$N=\left\{\left(t_{1},\left[p_{1}, p_{j}, \ldots ., p_{k}\right]\right) \mid t_{1} \varepsilon T\right.$ and $\left.p_{1}, p_{j}, \ldots \ldots, p_{k} \varepsilon P\right\}$

belng the token absorber set and $p_{1}, p_{j} \ldots p_{k}$ being the cancelled places.

C : 1s the control which associates with each transition a pair of logics, 1.e.; one of the possible combinations ( (AND, AND), (AND EOR), (EOR, AND), (BOR, EOR)\} .

The following topological restrictions are imposed. If $\left(p_{i}, t_{j}\right) \varepsilon I$, then $\left(t_{j}, p_{1}\right) t 0$. If $p_{1} \in S$ and $\left(p_{1}, t_{j}\right) \in I$, then no other switch is an Input place to $t_{j} ; t_{j}$ is elther of (AND, EOR) or (EOR, EOR) logic and there are only two places to $t_{f}$, the two output arcs leading from $t_{f}$, being labelled respectively $f$ and $e$.

In h1s paper, Baer states the following: "although the EOR and AND logics have sufficient properties to show the flow of control in algorithm, we introduce nevercheless a new type of place that we call switches." This statement indicates that the switch Petri nets are not really more powerful than Petri nets with mixed input, output logics. This is not true. In a previous report [3], we proved that extended Petri nets $\left(\mathrm{TN}_{\mathrm{EXT}}\right)$ were more powerful than ordinary Petri nets (TN) with respect to their capabilities of representing coordinations and in fact were complete, 1.e., they could represent all posstble types of interaction between a group of processes. Just as extended Petri nets were used to construct a central controller as powerful as a Turing machine, switch Petri nets can also be used to construct a simflar controller. This report is not the place to go into the details of such a construction but the interested reader can easily see how it is. accomplished by referring to our earlier 
Page 77

report. Thus we conclude that switch Petri nets ( $\mathrm{TN}_{\text {SWITCH}}$ ) are complete and $\mathrm{TN}_{\text {SWITCH }}=\mathrm{TN}_{\mathrm{EXT}}$.

\subsection{Other mode1s}

In 1970 Bredt and McCluskey proposed a model [8] where flow table methods from sequential circuit theory are applied to describe a control mechanism for asynchronous processes. The mechanism can be implemented either as a control circult or a control program but in either case, it represents a finite state control and is thus equivalent to $\mathrm{TN}_{\mathrm{SAFE}}$.

Extensions to Difkstra's $P$ and $V$ primitives [11] for synchronizing have been studied by Cerf [10]. He proposes PA, PX and V primitives. These are equivalent to the $\mathrm{CBD}$ model. The extended primitives under study by us are defined as follows:

$\operatorname{PE}\left(s_{1}, s_{2}, \ldots \ldots, s_{n}, s_{n+1}, \ldots . ., s_{n+m}\right)=\left[\right.$ if $s_{1}>0,1 \leq 1 \leq n$ and $s_{n+i}=0,1 \leq i \leq m$, then $s_{1}+s_{i}-1,1 \leq 1 \leq n$ else Wait $]$.

$v\left(s_{1}, \ldots, s_{n}\right)=\left[s_{1}+s_{1}+1,1 \leq i \leq n\right]$

As usual, these operations are "indivisible." It is easy to see that these primitives are equivalent to $\mathrm{TN}_{E X T}$ and therefore also complete.

\section{Summary and Conclusions}

The final hierarchy of nets and the relative positions of different controlling agents with respect to the hierarchy is given on page 78 and the abbreviations are explained on page 79. At the top of the hierarchy we have seven models. PE, V primitives, ASSM, ATMC, LGCS, GKMPS, TN and BSPN. Of these PE, $V$ primitives, $\mathrm{TN}_{E X T}$ and BSPN are probably the only ones that have significant practical usefulness in modelling and representing parallel systems. This will be the subject of a future techn1cal report. 
Page 78

As mentloned in the introduction, this report is a first step towards establishing a uniform underlying theory for the study of asynchronous systems. The results show the close relationship between varlous models. In the future, 1solated studies on different models should not be the direction taken by researchers. Rather, more effort should be directed towards establishing a theory for modelling, analyzing, synthesizing and understanding systems of asynchronous processes.

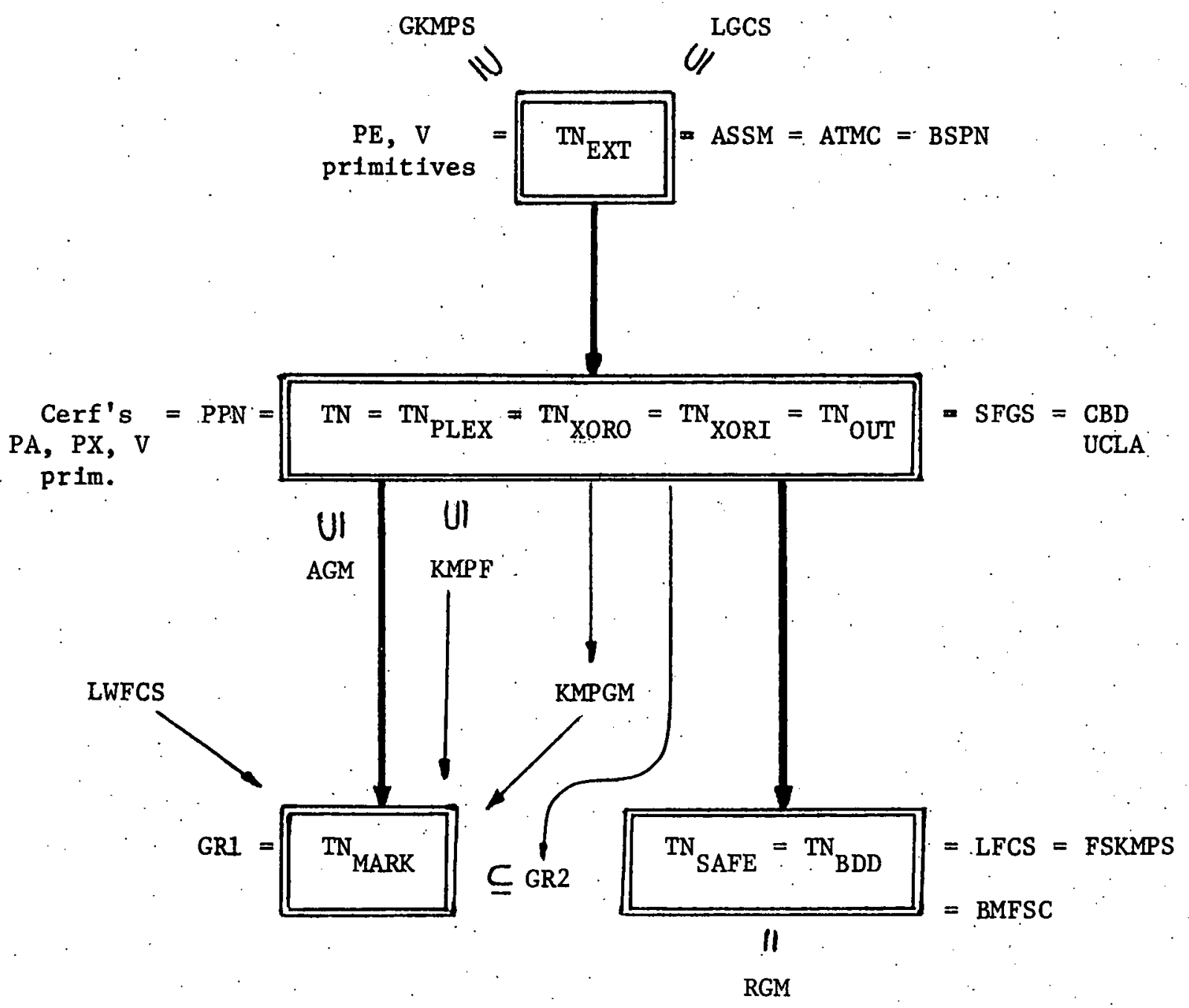


CBD

UCLA : Complex Bilogic Directed. Graph, UCLA

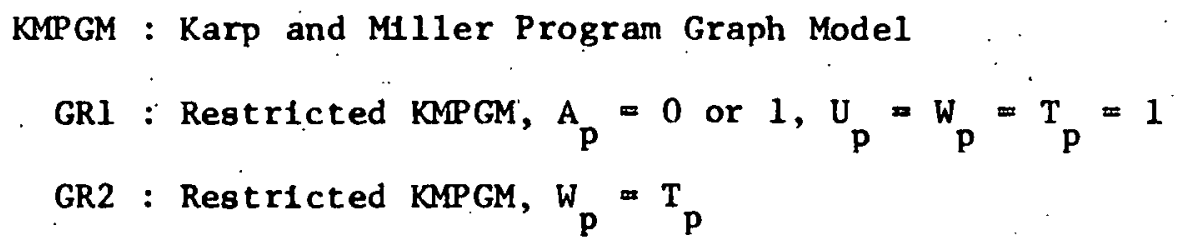

GKMPS : General Karp and Miller Parallel Program Schema

FSKMPS : Fintte State Karp and Miller Parallel Program Schema

KMPF : Karp and Miller Parallel Flow Charts

SFGS : Slutz Flow Graph Schema

LGCS : Luconi's General Computational Schema

LFGS : Luconf's Finfte State Computational Schema

LWFCS : Lucont's Well formed Computational Schema

AGM : Adam's Graph Model

RGM : Rodriguez' Graph Model

PPN : Peterson's P-Nets

BSPN : Baer's Switch Petri Nets

BMFSC : The Bredt and McCluskey Finfte State Control

ATMC : Agerwala's Turing Machine Control

ASSM : Agerwala's SS Model 
Page 80

\section{Acknowledgment}

I am grateful to my Advisor, Professor M.J. Flynn, for his guidance, encouragement and support during the course of this research.

\section{References}

1. Adams, D.A., "A model for parallel computations". Parallel Processor Systems, Technology and Applications, L.C. Hobbs et. al. (Eds), Spartan Books, N.Y. 1970.

2. Agerwala, T., "Comments on the capablifties, limitations and 'correctness' of Petri nets". Proceedings of the First Annual Symposium on Computer Architecture, Univers1ty of Florida, Dec. 9-11, 1973. A1so published as Hopkins Computer Research Report \# 26, Computer Science Program, The Johns Hopkins University, Baltimore, Md. 21218, July 1973.

3. Agerwala, T:, "A complete model for representing the coordination of asynchronous processes". Hopkins Computer Research Report \#32, Computer Science Program, The Johns Hopkins University, Baltimore, Md. 21218, July 1974

4. Agerwala, T., unpub11shed notes.

5. Baer, J.-L., "Graph models of computations in computer systems". Ph.D. dissertation, Rpt. \# 68-46, Department of Engineering, U.C.L.A., 1968.

6. Baer, J.-L., "A survey of some theoretical aspects of multiprocessing". ACM Computing Surveys, Vo1. 5, No. 1, March 1973.

7. Baer, J.-L., "Modelling for parallel computation: A case study". Proceedings of the 1973 Sagamore Computer Conference on Parallel Processing, Syracuse University, August 22-24, 1973.

8. Bredt, T.H. and.McCluskey, E.J., "Analysis and synthesis of control mechanlsms for parallel processes". Parallel Processor Systems Technologies and Applications, Hobbs, L.C. et. al. (Eds), Spartan Bks.., N.Y.1970. 
Page 81

9. Bovet, D.B., "Memory allocation in computer systems". Ph.D. dissertat1on, Rpt. \# 68-17, Department of Engineering, U.C.L.A., 1968.

10. Cerf, V.G., "Mult1processors, semaphores and a graph model of computation". Ph.D. dissertation, ENG-7223, Computer Science Department, U.C.L.A., Apr11 72.

11. Dijkstra, E.W., "Cooperating sequentlal processes". Programming Languagca, F. Geiluyy (Ed) Academic Press, N.Y. 1968.

12. Gostelow, K.P., "Flow of control, resource allocation and the proper termination of programs". Ph.D. dissertation, ENG-7179, Computer Science Department, U.C.L.A., December 1971.

13. Holt, A.W., and Commoner, F., "Events and conditions". Record of of the Project MAC Conference on Concurrent Systems and Parallel Computation, ACM, N.Y. 1970.

14. Karp, R.M. and Miller; R.E., "Properties of a model for parallel computations: determinacy, termination, queuing". SIAM Journal of Applied Mathematics, No. 14, November 1966.

15. Karp, R.M. and Miller, R.E., "Parallel program schemata". Journal of Computer and System Sciences, No. 3, 1969.

16. Luconi, F.L.,."Asynchronous computational structures".'Ph.D. dissertation, M.I.T., 1968.

17. Martin, D.F. "The automatic assignment and sequencing of computations on parallel processor systems". Ph.D. dissertation, Rpt.\# 66-4, Department of Englneering, U.C.L.A., January 1966.

18. Miller, R.E., "A comparison of some theoretical models of paralle1 computation". IEEE-TC, Vo1. C-22, No. 8, August 1973. 
19. Peterson, J.L., "The P-net as a model of parallel systems". Unpublished manuscript, Digital Systems Lab., Stanford Unfversity, Nov. 1973.

20. Petr1, C.A., "Communication with automata". Supplement 1 to Technical Report. RADC-TR-65-377, Vo1. 1, Griffiss AF Base, N.Y. 1966.

21. Rodriguez, J.E., "A graph model for parallel computations". Ph.D. dissertation, M.I.T., 1967.

22. Russe1, E.C., "Automat1c program analysis". Ph.D. dissertation, Rpt. \#. 69-12, Department of Engtneering, U.C.L.A., August 1973.

23. Slutz, D.R.; "Flow graph schemata". Record of the Profect MAC Conference on Concurrent Systems and Parallel Computation, ACM, N.Y., 1970

24. Volansky, S.A., "Graph model analysis and Implementation of computational sequences". Rpt. \#63-5, Department of Engineering, U.C.L.A., June 1970. 
Page 83

NOTE 1

For, theoretical questions concerning capabilities, limitations; etc. this assumption is valid. It essentially amounts to saying that one further restriction has to be placed in the simulation rules, i.e., only one transition at a time is allowed to fire. This does not mean that the net cannot represent asynchrony in the theoretical context. A process

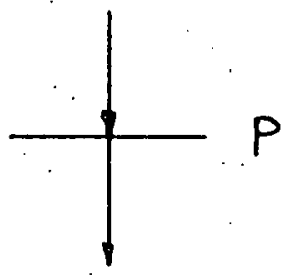

can easily be represented as

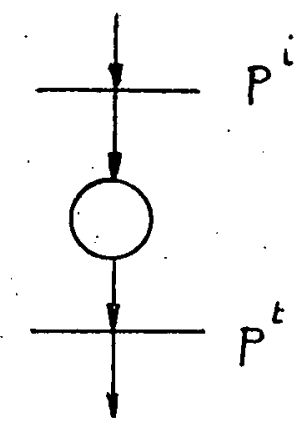

$\mathrm{P}^{\mathrm{i}}$ indicates initiation and $\mathrm{p}^{\mathrm{t}}$, termination. . Now, the rule that transitions cannot fire at the same time simply reduces to the rule that initiations and terminations do not occur at the same instant. Processes that have initiated, but not terminated, are running and a number of processes may be running at the same time, thus representing asynchronous operation. Thus the assumption does not affect the modelling power of a net: In a net used to represent some practical problem, the restriction may be removed. Even so, we do assume that removal of markers from input places and placement of markers in output places by different transitions do not occur at the same time. Also, in a practical situation one would normally want well behaved nets. 
Page 84

NOTE 2

Important note introduced at time of publication

It has been brought to my notice that J.L. Peterson and T.H. Bredt have performed a similar comparison of models of computations in their paper "A comparison of models of parallel computations" preprints, IFIP Congress 1974, August 5-10, 1974, Stockholm, Sweden. The portion of their results that overlap with those presented here is glven below:

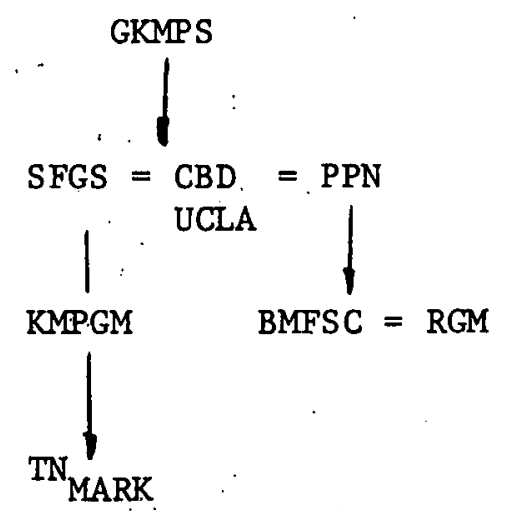

In addition, they also consider Vector Addition Systems, Vector Replacement Systems, T-counter Transition Systems, Coordination Nets, Finishing Schemata, and Message Transmission Systems. The major differences are that. their paper is less formal and spectifc thau thio ono. They do not discuss restricted subclasses within a particular model. The extended Petri nets and Baer's Switch Petri nets are not discussed and we believe both these models are exceedingly important because of their completeness and practical usefulness. Our results also indicate that multiple arcs between a place and a transition do not provide any extra power since $\mathrm{TN}=\mathrm{PPN}$. Also, the generalized primitives are not mentioned. Overall, the major results are remarkably similar. Both papers establish the 
Page 85

need for a untform underlying theory of models for parallel systems and provide the first steps in this direction. 